\title{
Construction of [6-5-5-6-6] Pentacyclic Skeleton via Phosphine Catalyzed Domino Reaction and Mechanism Study
}

Shanshan Ma, ${ }^{\dagger}$ Aimin $\mathrm{Yu},{ }^{\dagger}$ Shunguang Zhang, ${ }^{*}, \dagger$ Lei Zhang $*, \neq, \S$ and Xiangtai Meng $*, \dagger$

†Tianjin Key Laboratory of Organic Solar Cells and Photochemical Conversion; Tianjin Key Laboratory of Drug Targeting and Bioimaging, School of Chemistry \& Chemical Engineering Tianjin University of Technology, Tianjin 300384, P.R. China

Tianjin Engineering Technology Center of Chemical Wastewater Source Reduction and Recycling, School of Science Tianjin Chengjian University, Tianjin 300384, P.R. China

${ }^{\S}$ College of Chemistry, Beijing Normal University, Beijing 100875, P. R. China

\section{Table of Contents}

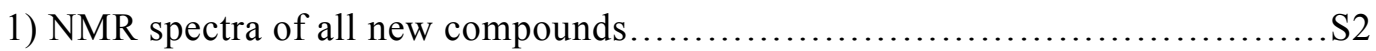

2) Computational details and archive entries......................................... 58

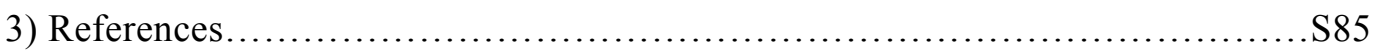

4) X-ray data collection and structure determinations......................... 85

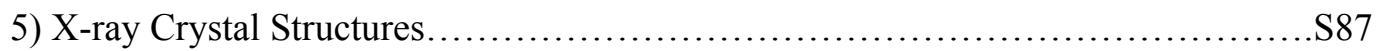

6) Some equations in chemical kinetics to determine the difference of the free-energy barriers of two competing reactions from the product ratio observed.....................S87 


\section{NMR spectra of all new compounds}
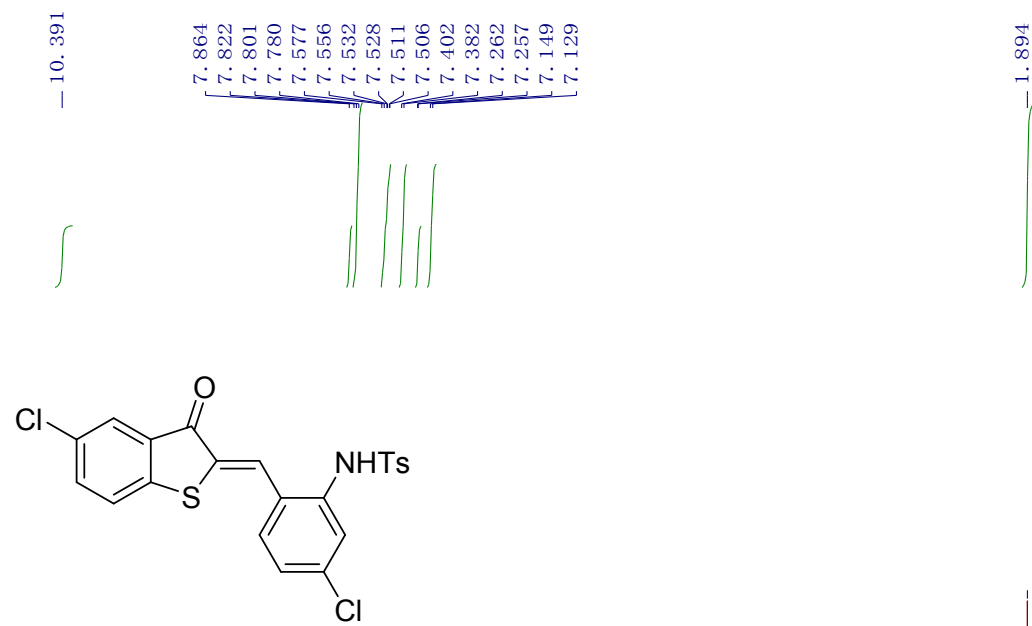

1b, ${ }^{1} \mathrm{H}$ NMR $400 \mathrm{MHz}$, DMSO
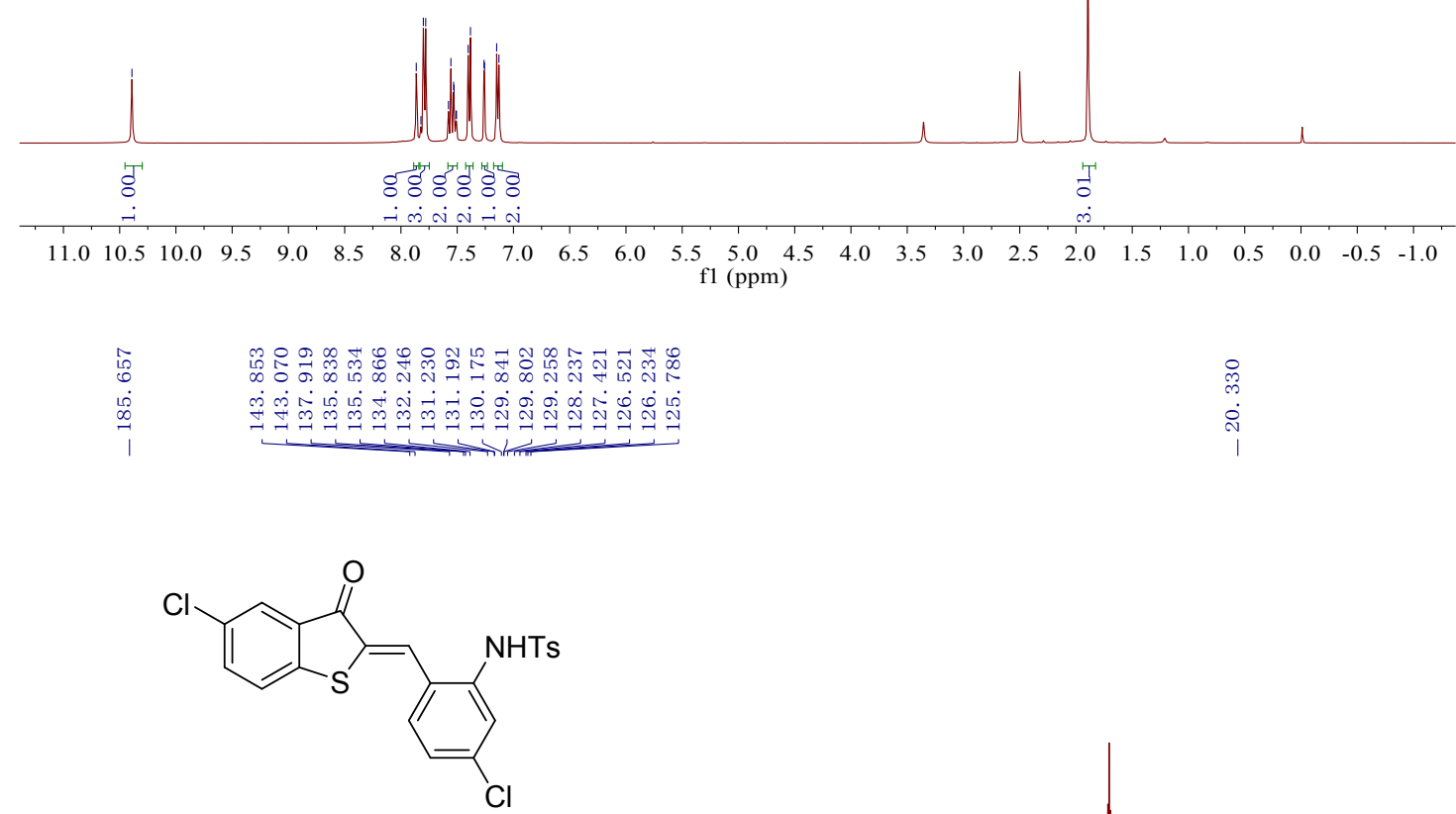

1b, ${ }^{13} \mathrm{C}$ NMR $100 \mathrm{MHz}$, DMSO

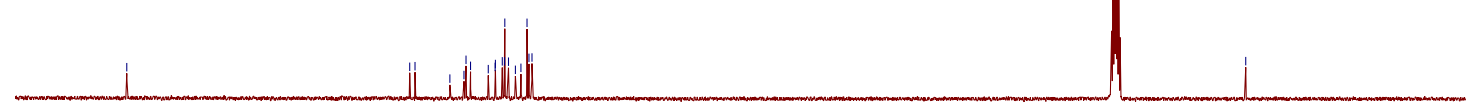

$\begin{array}{lllllllllllllllllllll}200 & 190 & 180 & 170 & 160 & 150 & 140 & 130 & 120 & 110 & \begin{aligned} 100 \\ \mathrm{f} 1(\mathrm{ppm})\end{aligned} & 80 & 70 & 60 & 50 & 40 & 30 & 20 & 10 & 0 & -1(\end{array}$ 

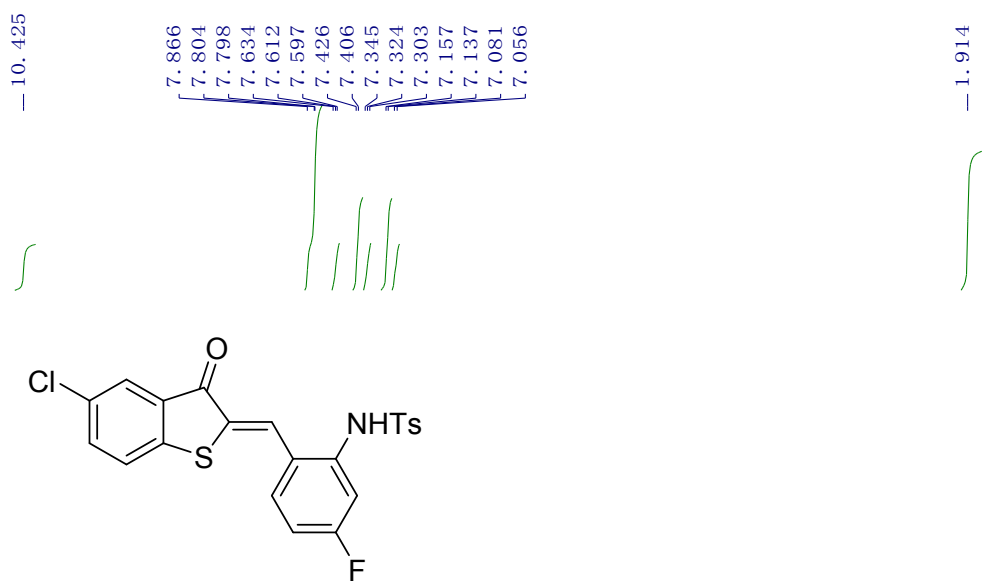

Ic, ${ }^{1} \mathrm{H}$ NMR $400 \mathrm{MHz}$, DMSO
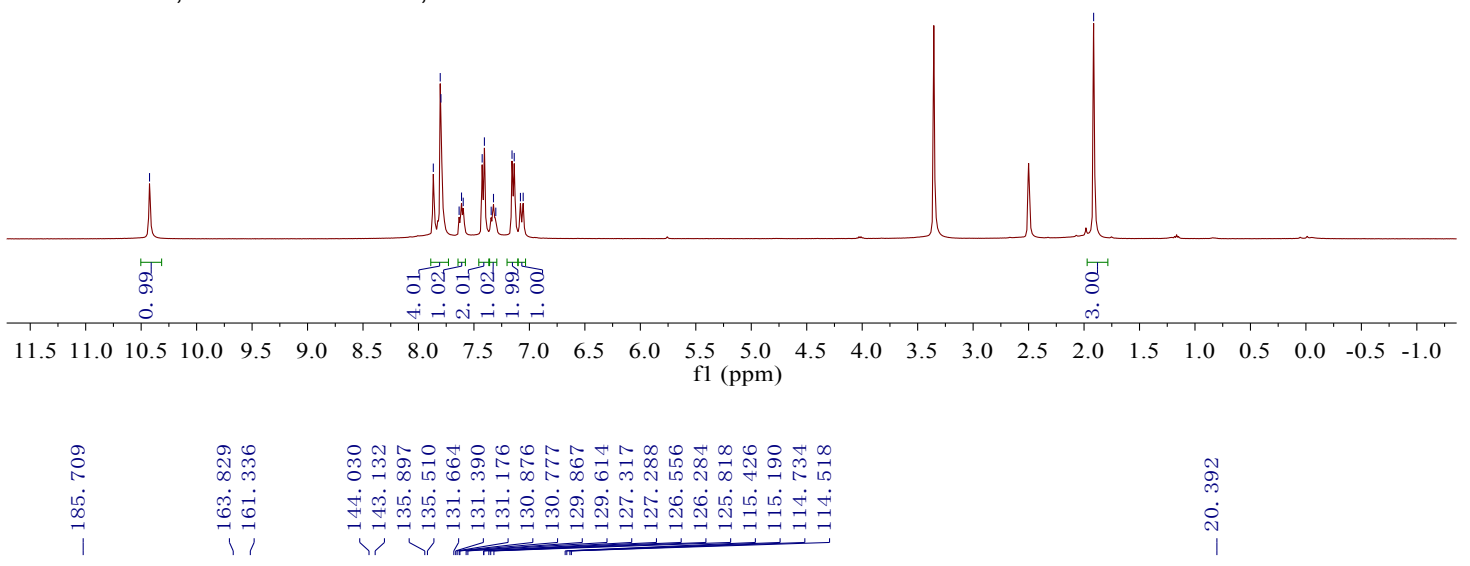

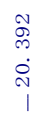
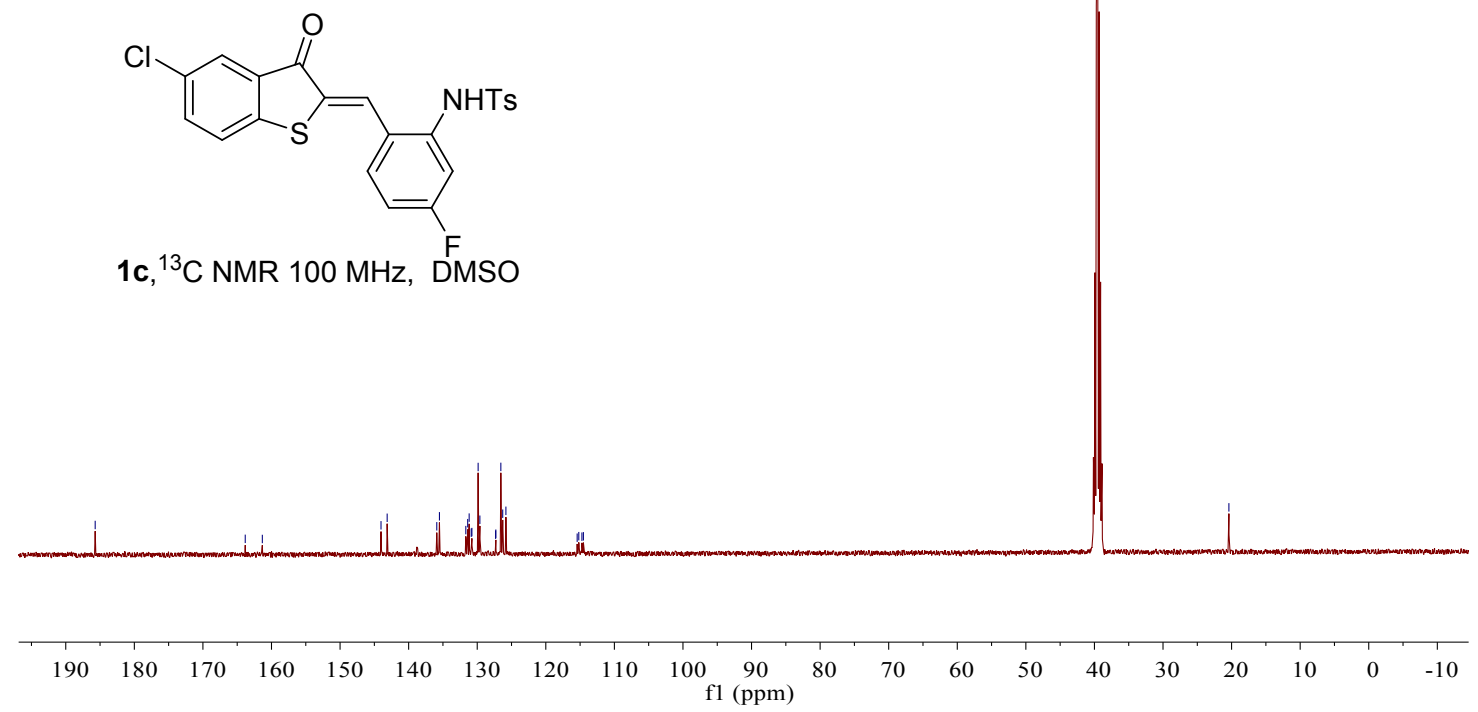

SB 

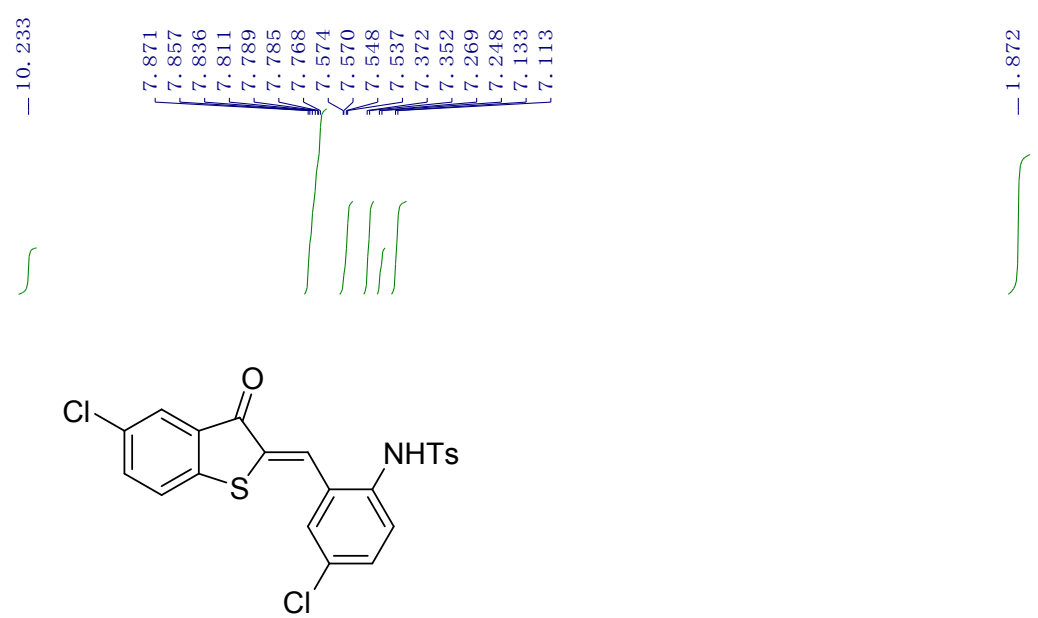

1d, ${ }^{1} \mathrm{H}$ NMR $400 \mathrm{MHz}$, DMSO
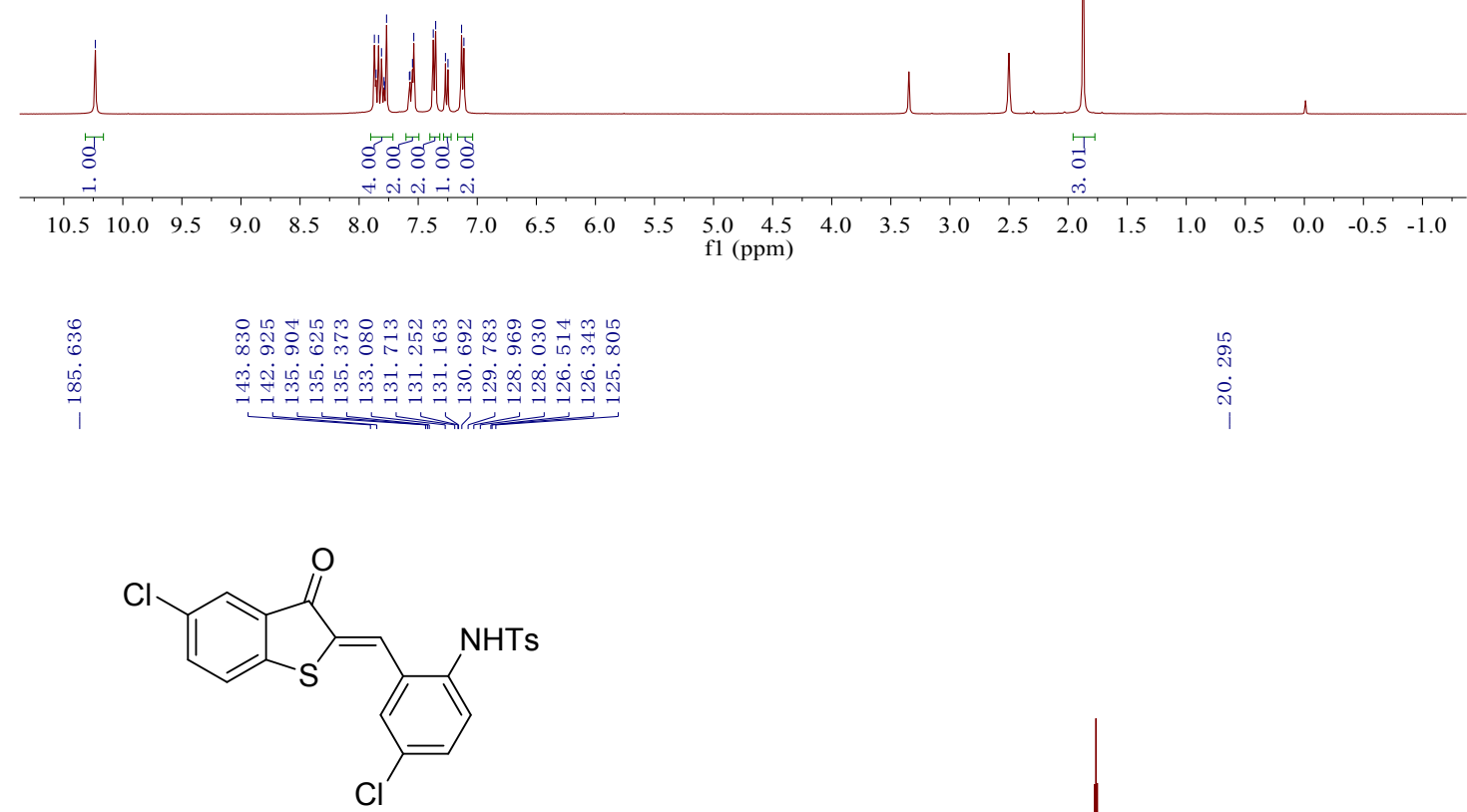

1d, ${ }^{13} \mathrm{C}$ NMR $100 \mathrm{MHz}$, DMSO

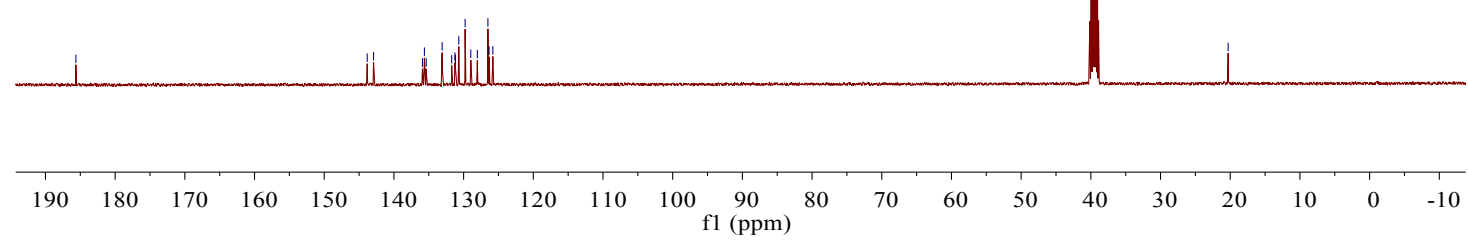




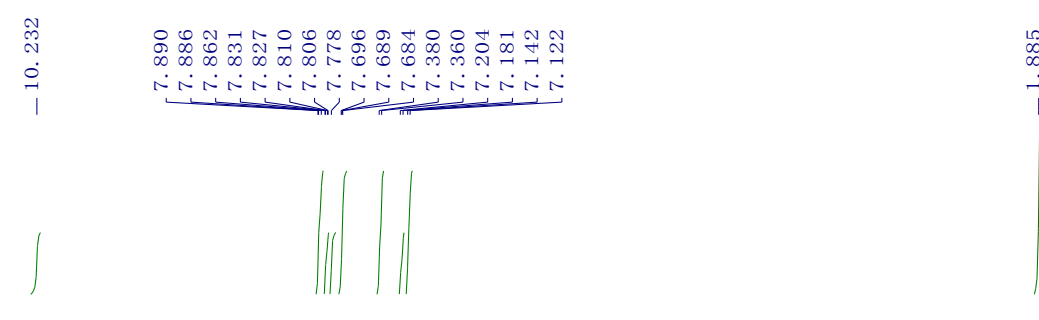<smiles>O=C1/C(=C/c2cc(Br)ccc2N[I-])Sc2ccc(Cl)cc21</smiles>

1e, ${ }^{1} \mathrm{H}$ NMR $400 \mathrm{MHz}$, DMSO

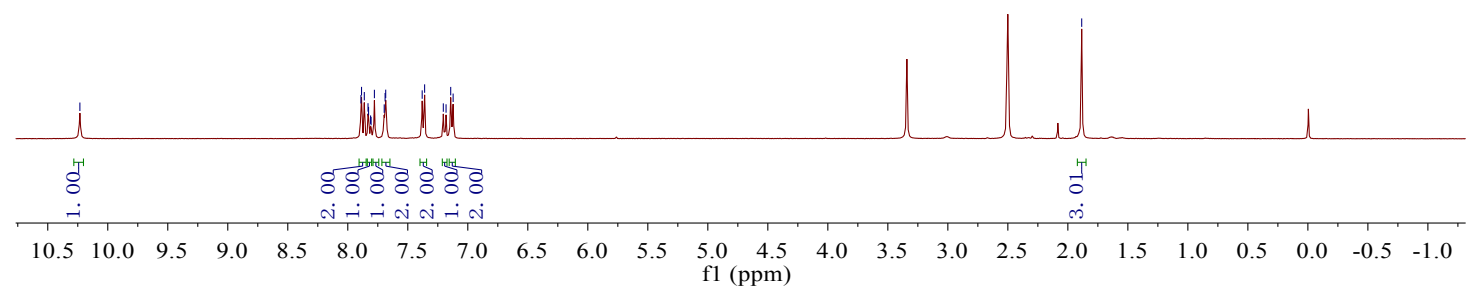

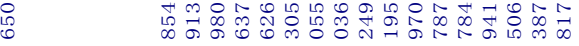

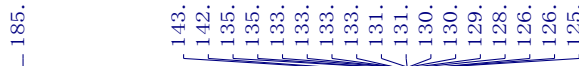<smiles>Nc1ccc(Br)cc1/C=C1\Sc2ccc(F)cc2C1=O</smiles>

1e, ${ }^{13} \mathrm{C}$ NMR $100 \mathrm{MHz}$, DMSO

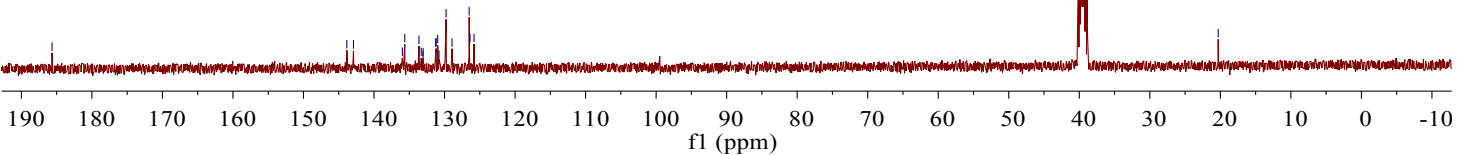



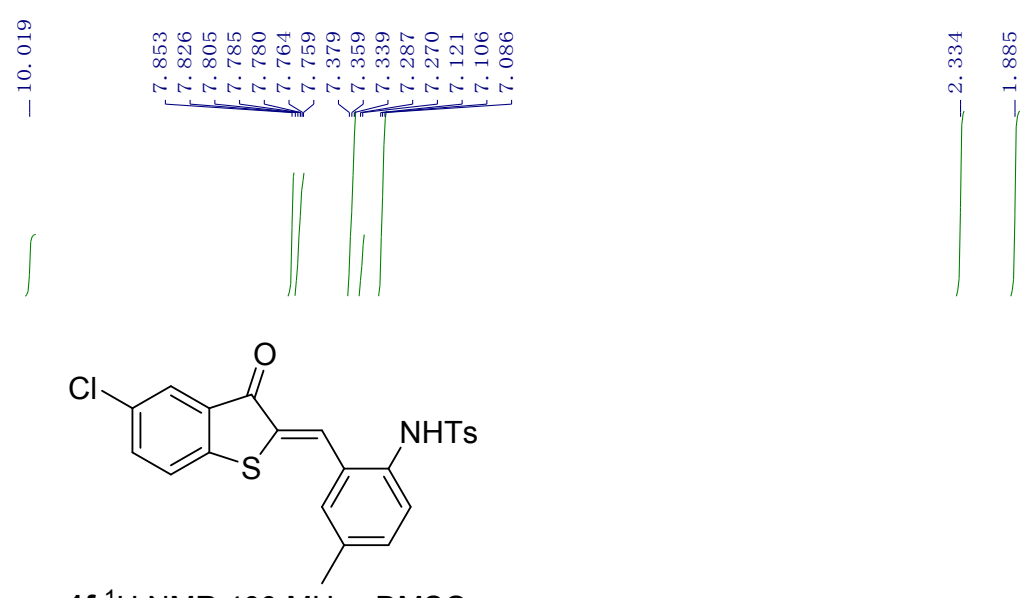

1f, ${ }^{1} \mathrm{H}$ NMR $400 \mathrm{MHz}$, DMSO

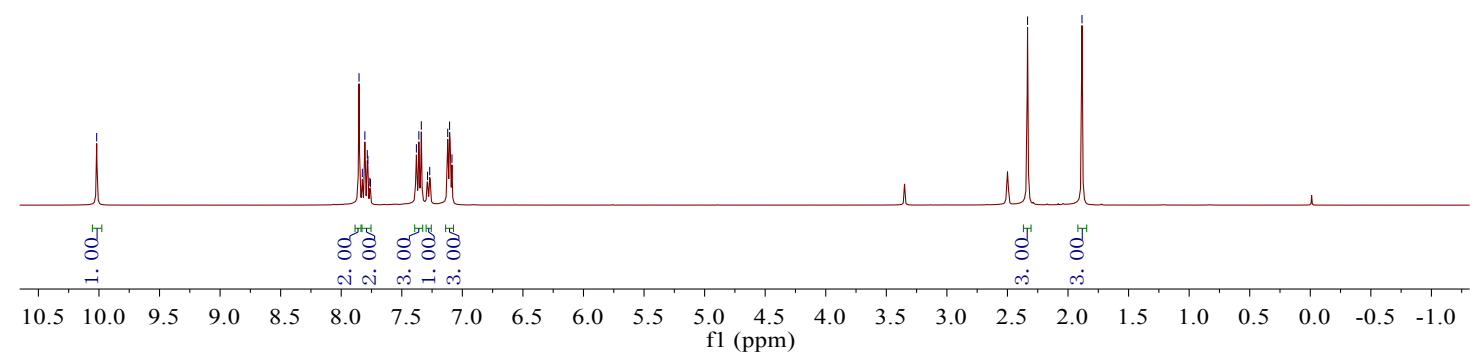

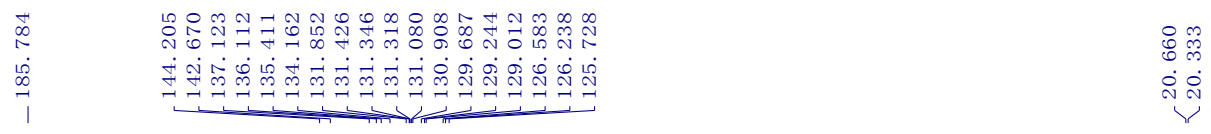<smiles>Cc1ccc(NS)c(/C=C2\Sc3ccc(Cl)cc3C2=O)c1</smiles>

1f, ${ }^{13} \mathrm{C}$ NMR $100 \mathrm{MHz}$, DMSO

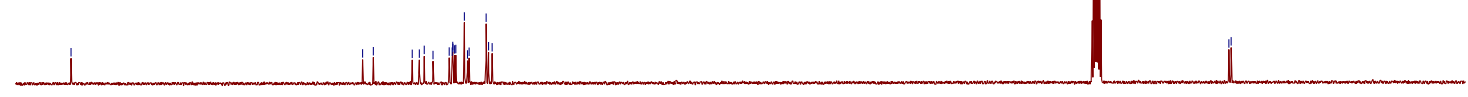

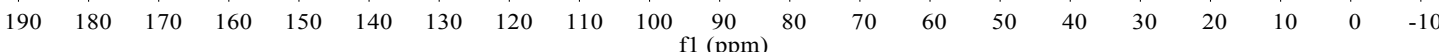




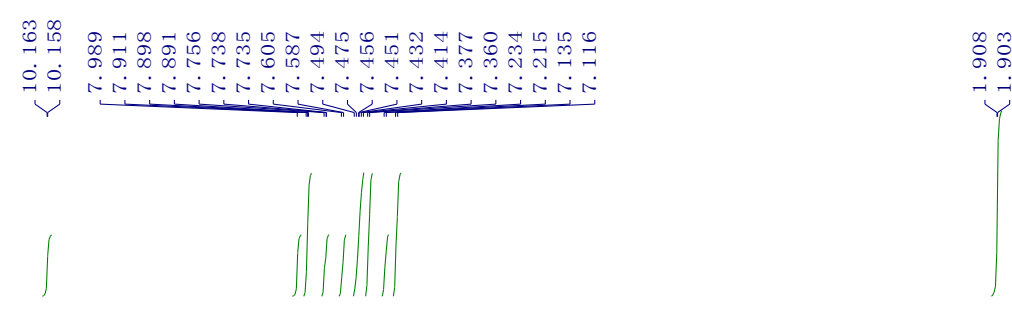<smiles>O=C1/C(=C/c2ccccc2N[AsH3])Sc2ccc(Br)cc21</smiles>

1g, ${ }^{1} \mathrm{H}$ NMR $400 \mathrm{MHz}$, DMSO

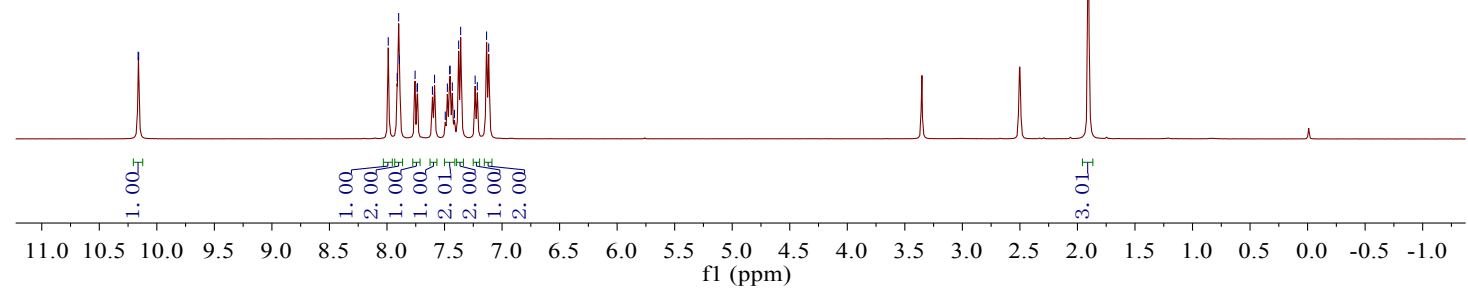

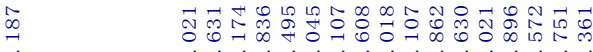

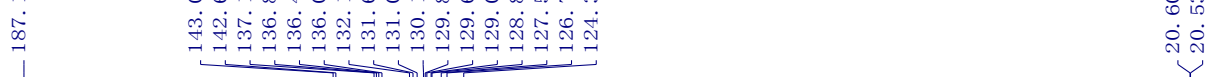<smiles>O=C1/C(=C/c2ccccc2N[AsH3])Sc2ccc(Br)cc21</smiles>

1g, ${ }^{13} \mathrm{C}$ NMR $100 \mathrm{MHz}$, DMSO

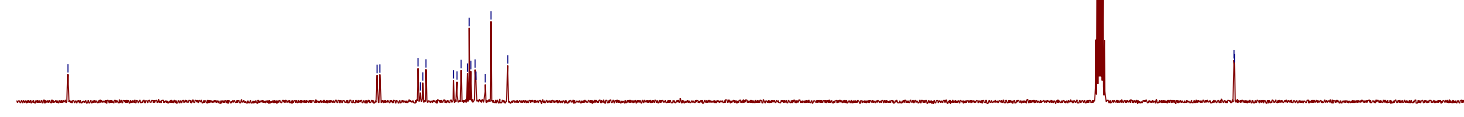

$\begin{array}{llllllllllllllllllllllll}190 & 180 & 170 & 160 & 150 & 140 & 130 & 120 & 110 & 100 & 90 & 80 & 70 & 60 & 50 & 40 & 30 & 20 & 10 & 0 & -10\end{array}$ 


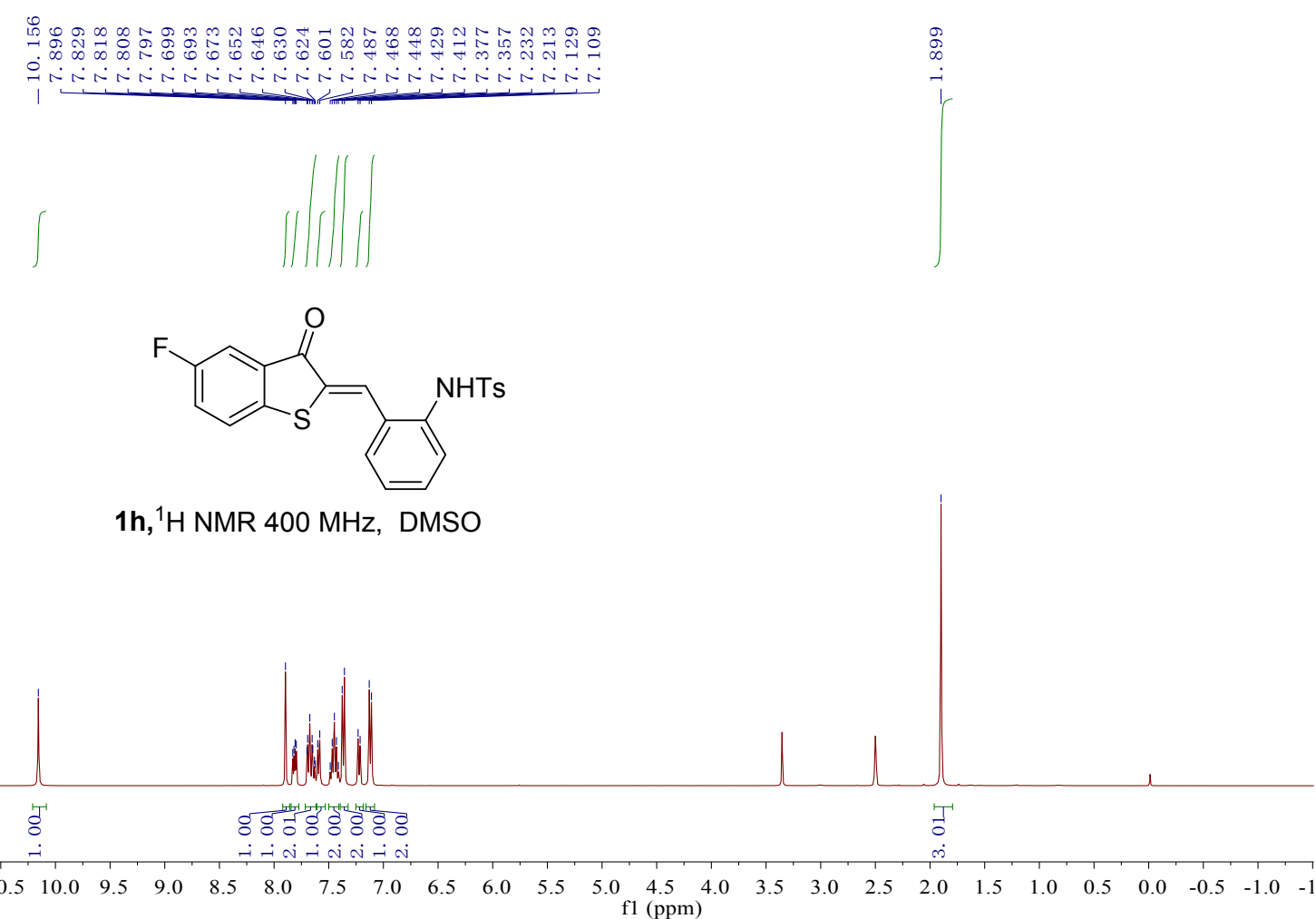

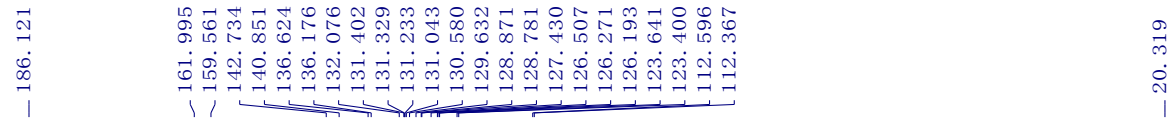<smiles>[B]Nc1ccccc1/C=C1\Sc2ccc(F)cc2C1=O</smiles>

1h, ${ }^{13} \mathrm{C}$ NMR $100 \mathrm{MHz}$, DMSO

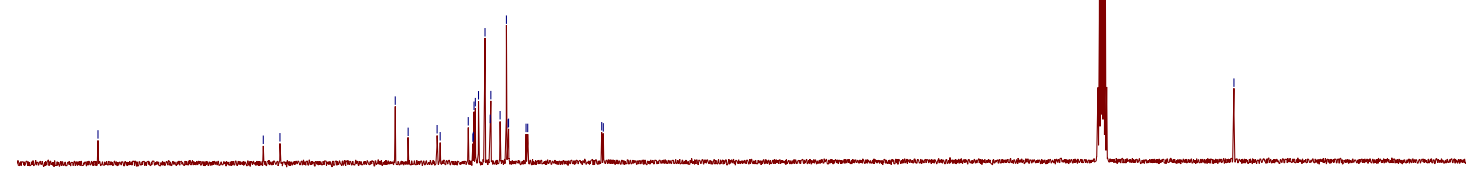

$\begin{array}{lllllllllllllllllllll}190 & 180 & 170 & 160 & 150 & 140 & 130 & 120 & 110 & 100 & 90 & 80 & 70 & 60 & 50 & 40 & 30 & 20 & 10 & 0 & -10\end{array}$ 


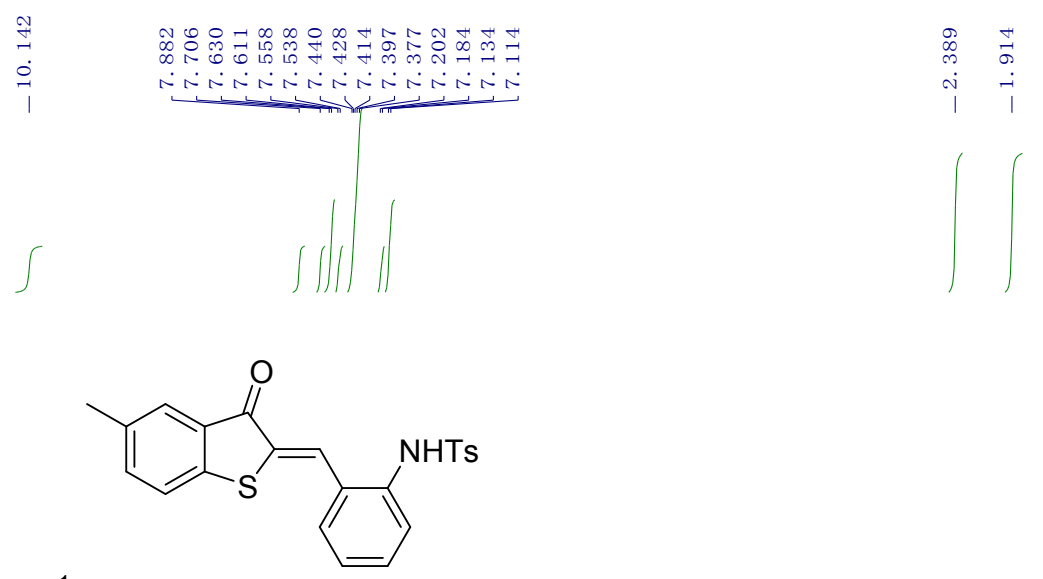

1i, ${ }^{1} \mathrm{H}$ NMR $400 \mathrm{MHz}$, DMSO

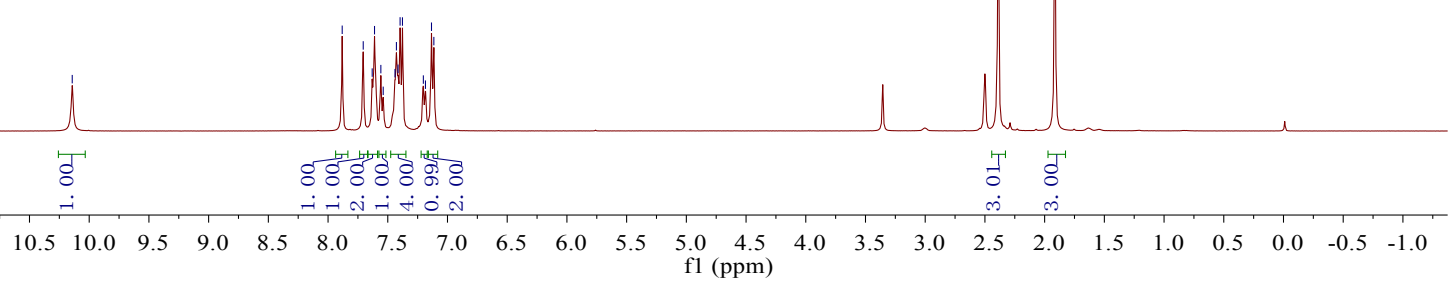

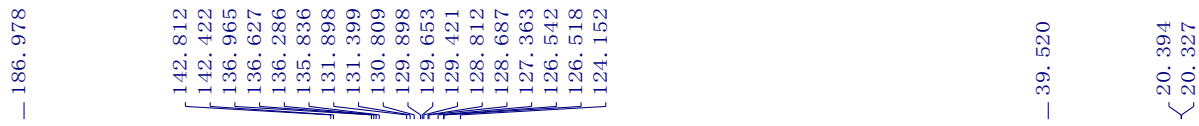<smiles>Cc1ccc2c(c1)C(=O)/C(=C/c1ccccc1NC(F)(F)F)S2</smiles>

1i, ${ }^{13} \mathrm{C}$ NMR $100 \mathrm{MHz}$, DMSO

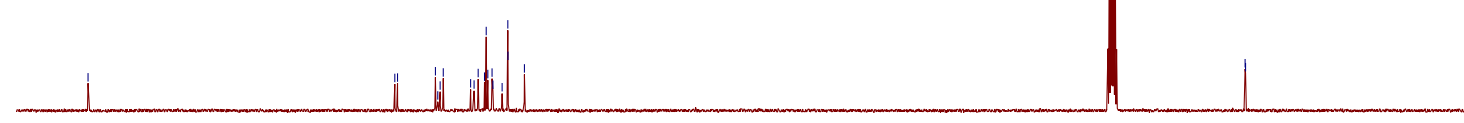

$\begin{array}{lllllllllllllllllllll}190 & 180 & 170 & 160 & 150 & 140 & 130 & 120 & 110 & 100 & \underset{90}{90} & 80 & 70 & 60 & 50 & 40 & 30 & 20 & 10 & 0 & -1\end{array}$ 


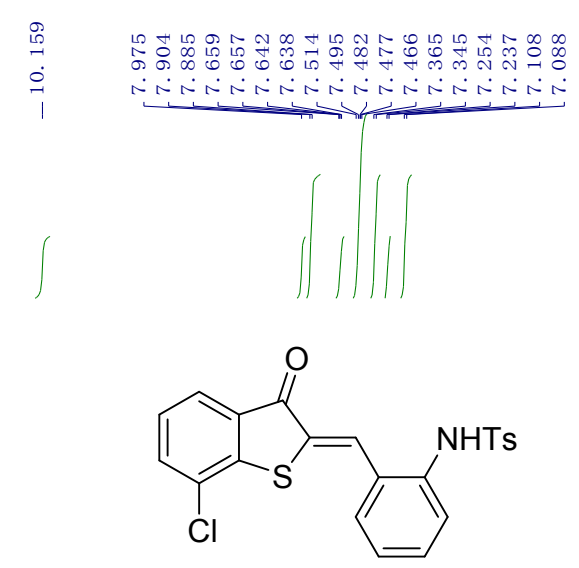

1j, ${ }^{1} \mathrm{H}$ NMR $400 \mathrm{MHz}$, DMSO

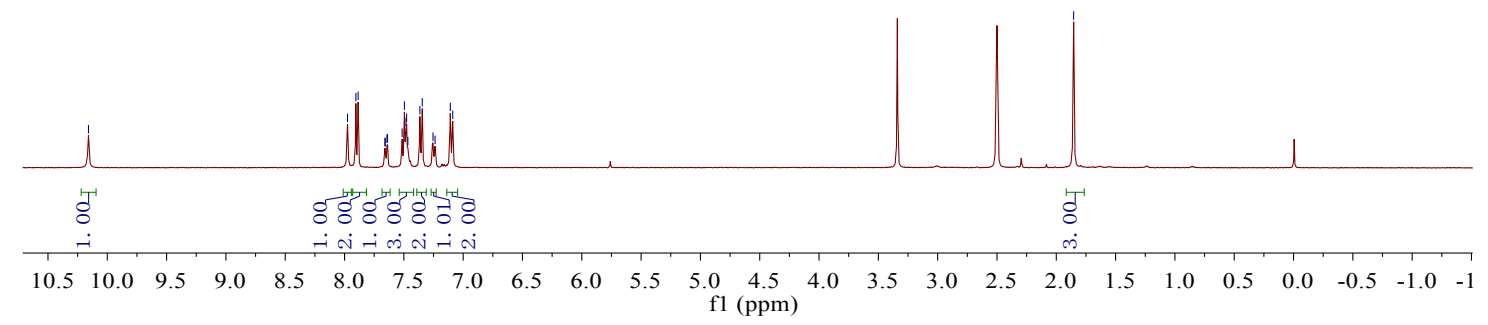

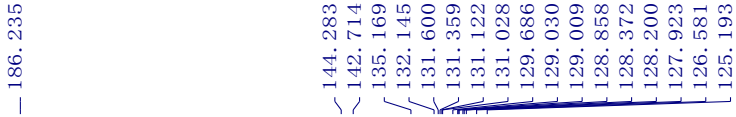

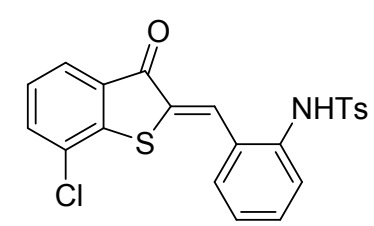

1j, ${ }^{13} \mathrm{C}$ NMR $100 \mathrm{MHz}$, DMSO

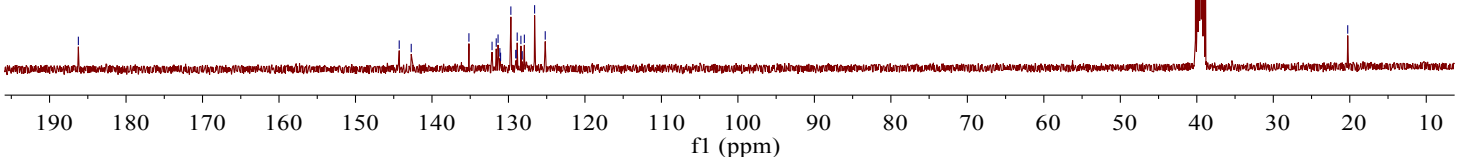




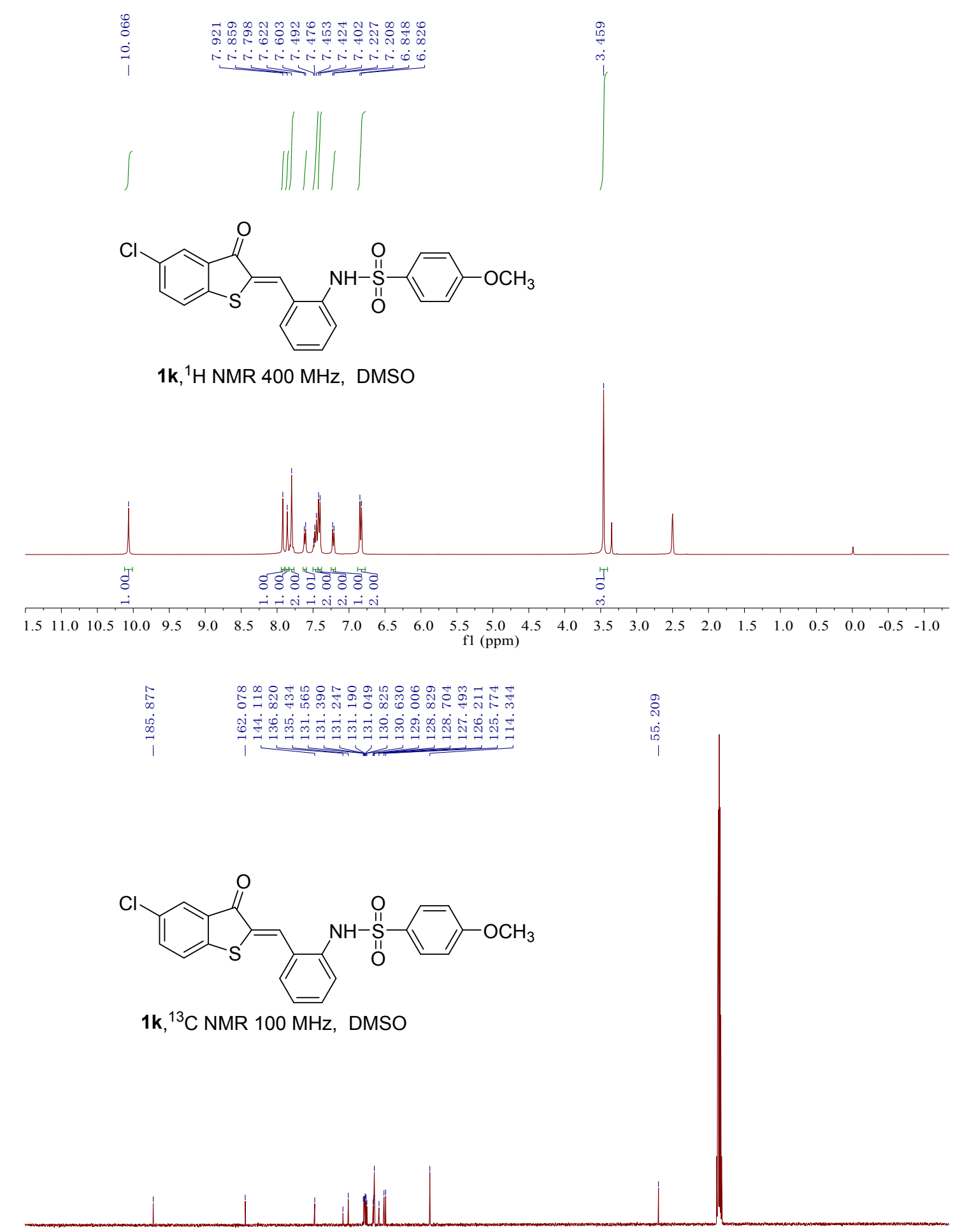

$\begin{array}{lllllllllllllllllllllll}210 & 200 & 190 & 180 & 170 & 160 & 150 & 140 & 130 & 120 & 110 & 100 & 90 & 80 & 70 & 60 & 50 & 40 & 30 & 20 & 10 & 0 & -10\end{array}$ 


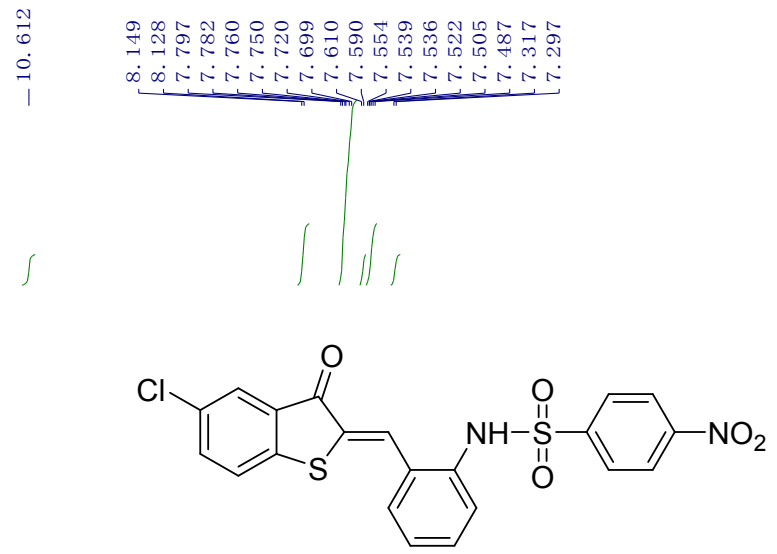

1I, ${ }^{1} \mathrm{H}$ NMR $400 \mathrm{MHz}$, DMSO

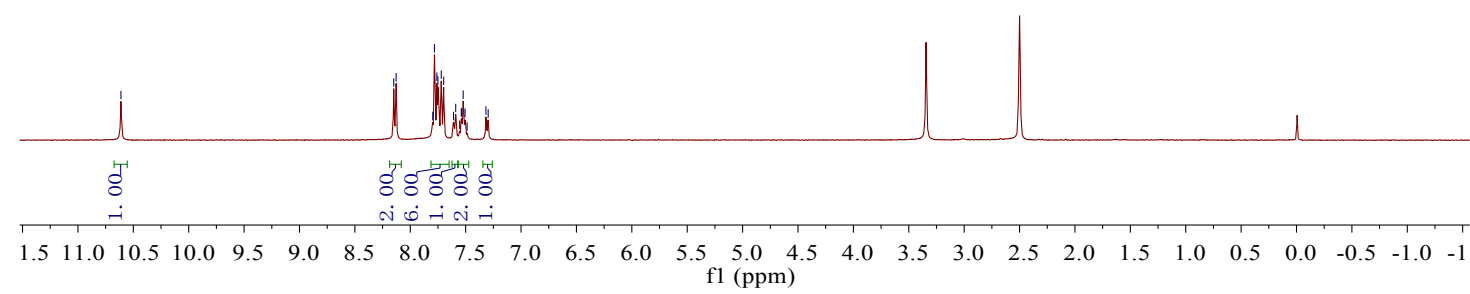

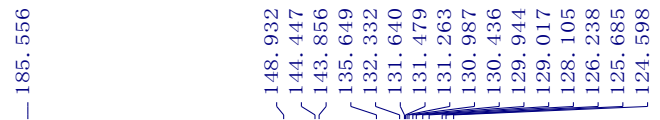<smiles>O=C1/C(=C/c2ccccc2NS(=O)(=O)c2ccc([N+](=O)[O-])cc2)Sc2ccc(Cl)cc21</smiles>

$1{ }^{13}{ }^{13} \mathrm{C}$ NMR $100 \mathrm{MHz}$, DMSO

$\begin{array}{lllllllllllllllllllll}190 & 180 & 170 & 160 & 150 & 140 & 130 & 120 & 110 & 100 & \begin{array}{l}90 \\ \mathrm{fl}(\mathrm{pm})\end{array} & 80 & 70 & 60 & 50 & 40 & 30 & 20 & 10 & 0 & -10\end{array}$ 


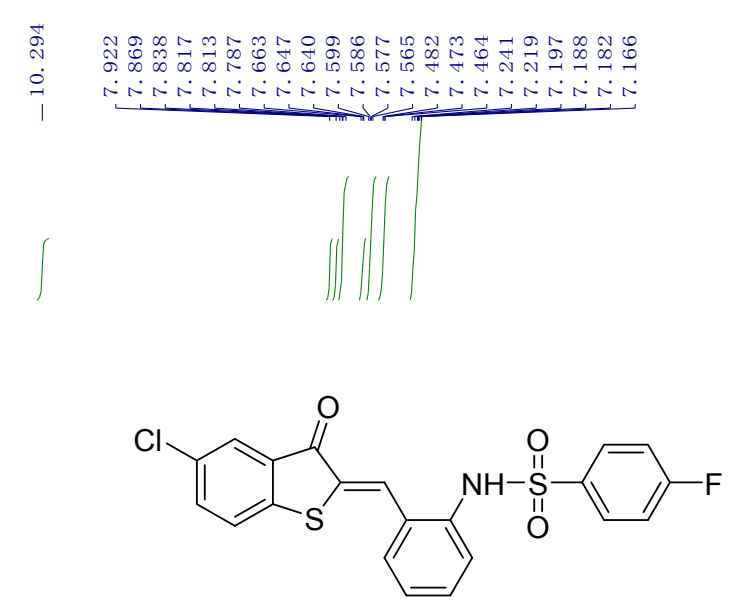

1m, ${ }^{1} \mathrm{H}$ NMR $400 \mathrm{MHz}$, DMSO

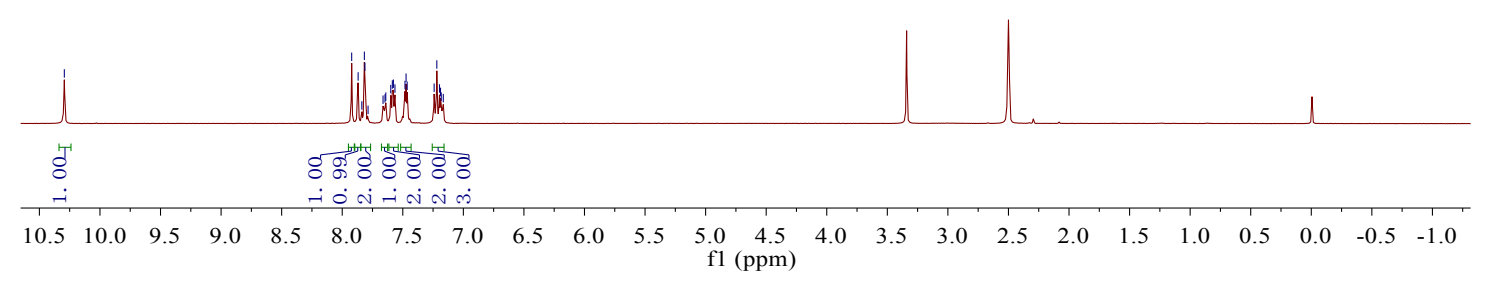

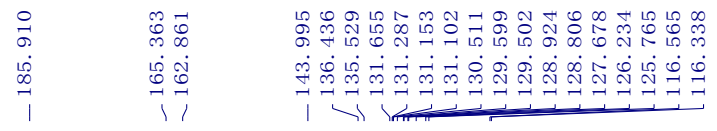

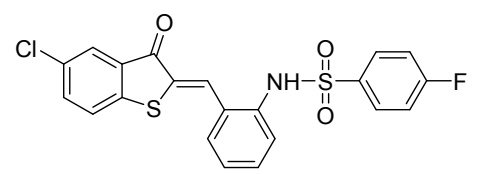

$1 \mathrm{~m},{ }^{13} \mathrm{C}$ NMR $100 \mathrm{MHz}$, DMSO

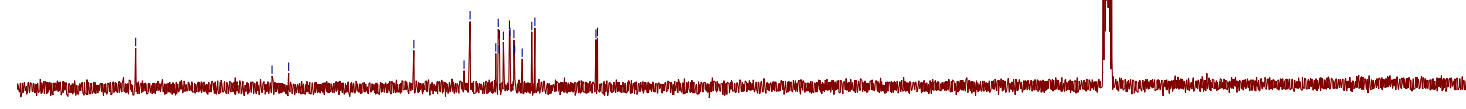

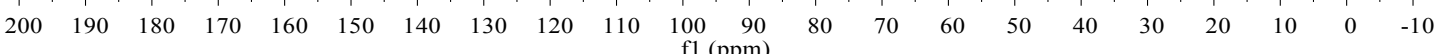




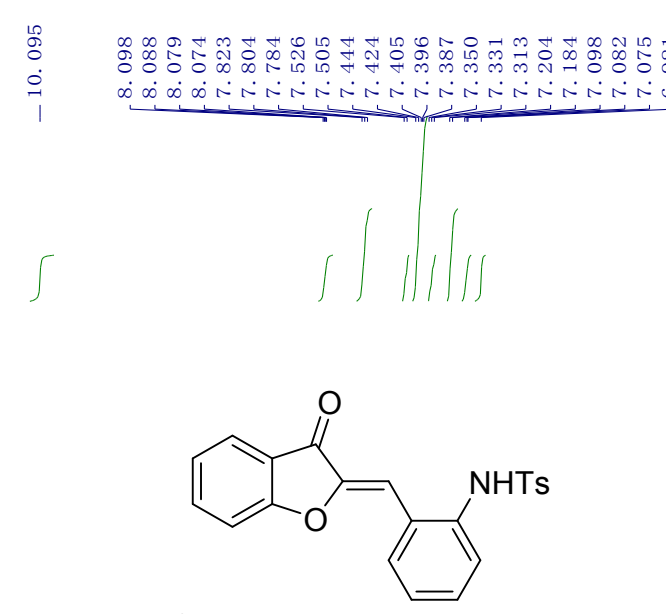

5a, ${ }^{1} \mathrm{H}$ NMR $400 \mathrm{MHz}$, DMSO

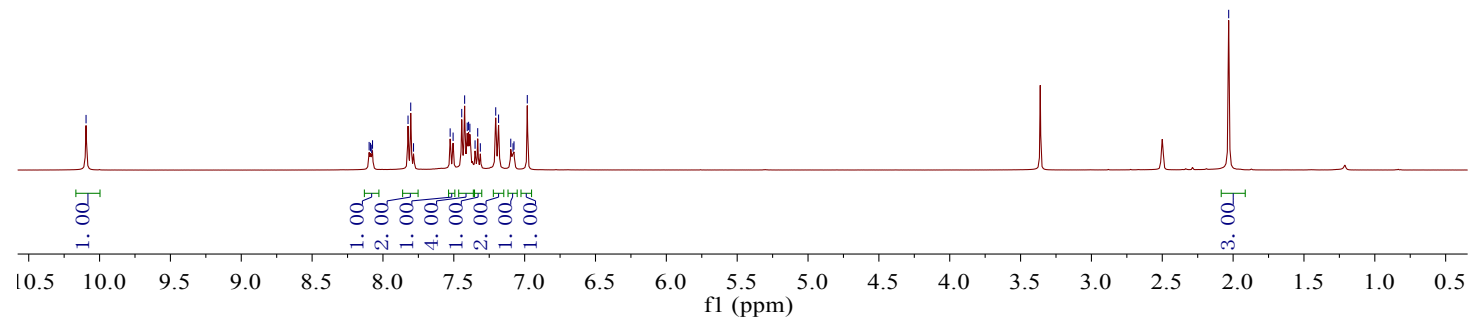

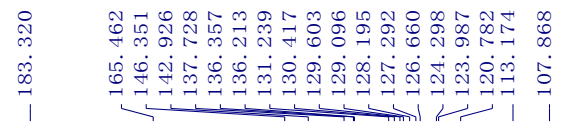<smiles>O=C1/C(=C/c2ccccc2NC(F)(F)F)Oc2ccccc21</smiles>

5a, ${ }^{13} \mathrm{C}$ NMR $100 \mathrm{MHz}$, DMSO

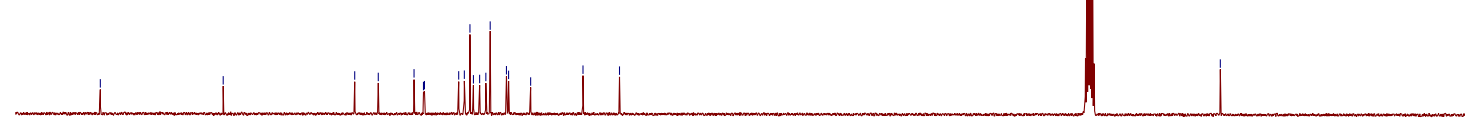

$\begin{array}{lllllllllllllllllllllll}190 & 180 & 170 & 160 & 150 & 140 & 130 & 120 & 110 & 100 & 90 & 80 & 70 & 60 & 50 & 40 & 30 & 20 & 10 & 0 & -10\end{array}$ 

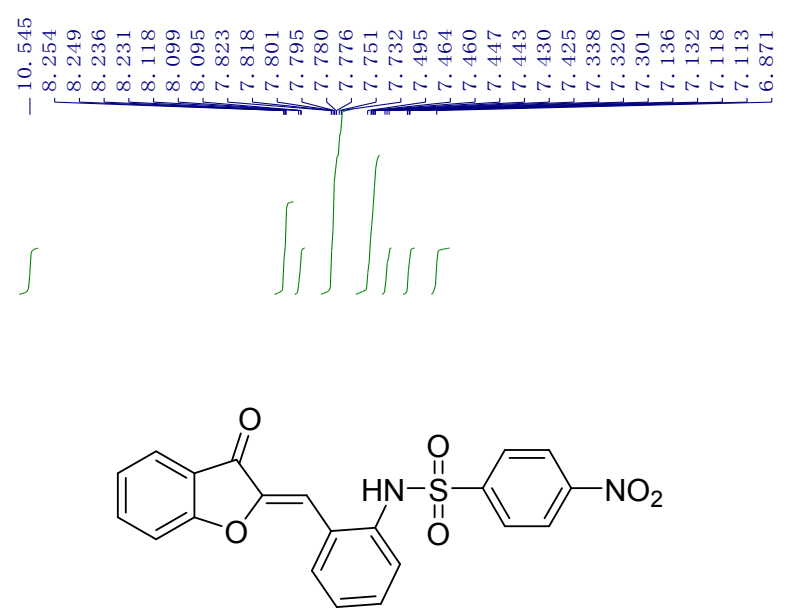

5b, ${ }^{1} \mathrm{H}$ NMR $400 \mathrm{MHz}$, DMSO

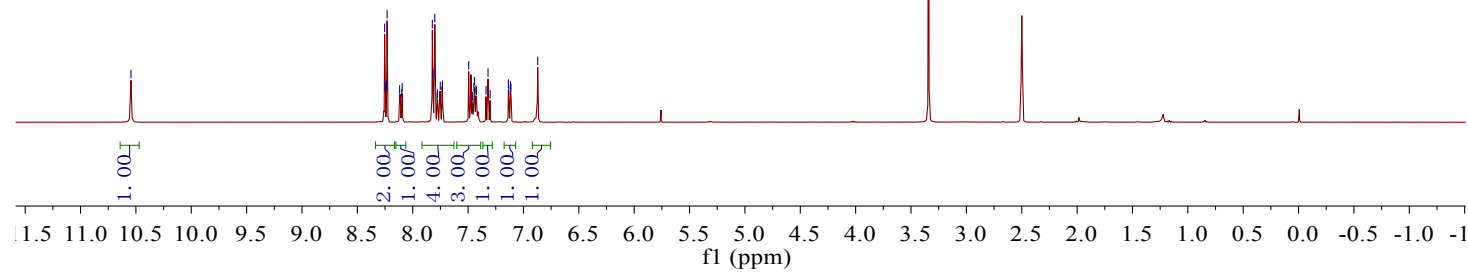

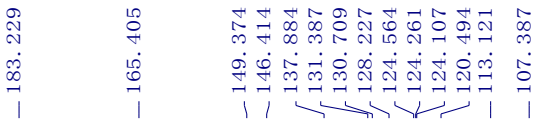

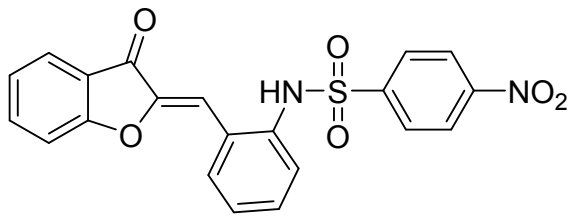

5b, ${ }^{13} \mathrm{C}$ NMR $100 \mathrm{MHz}$, DMSO

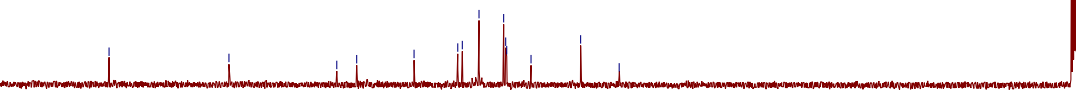

$\begin{array}{lllllllllllllllllllll}190 & 180 & 170 & 160 & 150 & 140 & 130 & 120 & 110 & 100 & 90 & 80 & 70 & 60 & 50 & 40 & 30 & 20 & 10 & 0 & -10\end{array}$ 


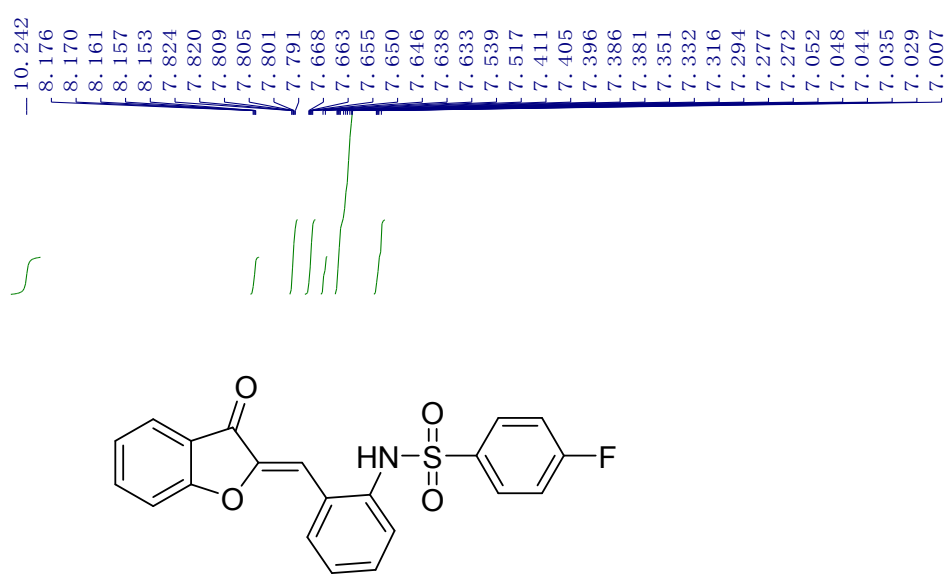

5c, ${ }^{1} \mathrm{H}$ NMR $400 \mathrm{MHz}$, DMSO

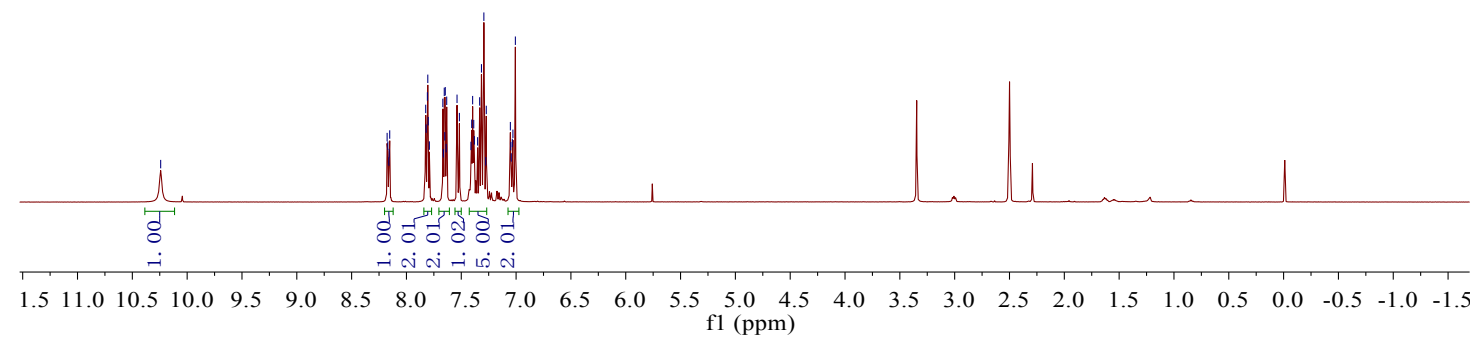

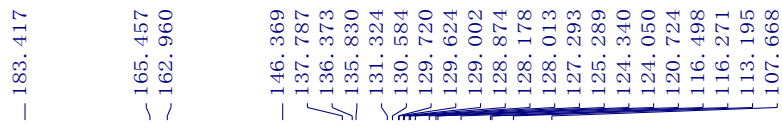

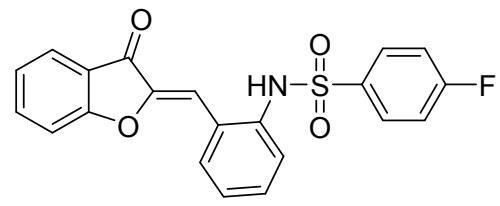

5c, ${ }^{13} \mathrm{C}$ NMR $100 \mathrm{MHz}$, DMSO

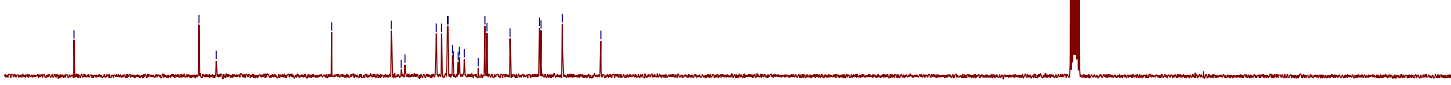

$\begin{array}{lllllllllllllllllllll}190 & 180 & 170 & 160 & 150 & 140 & 130 & 120 & 110 & 100 & 90 & 80 & 70 & 60 & 50 & 40 & 30 & 20 & 10 & 0 & -10\end{array}$ 


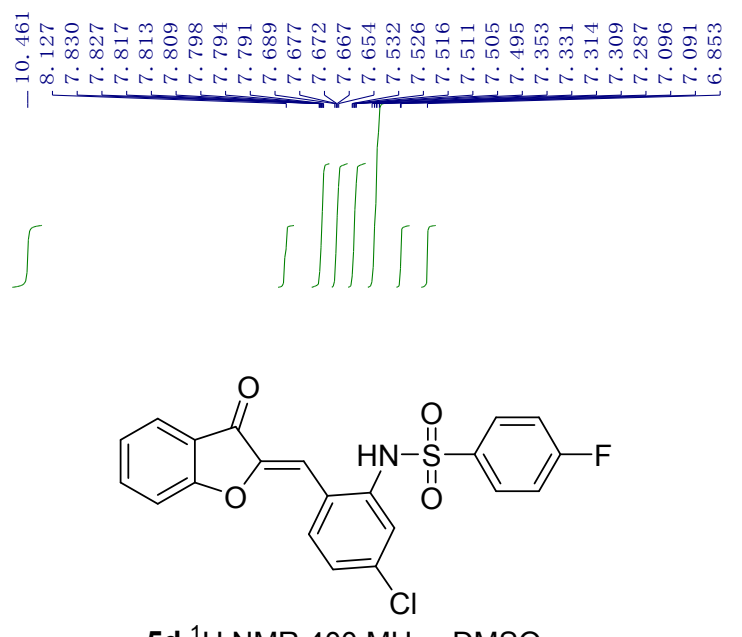

5d, ${ }^{1} \mathrm{H}$ NMR $400 \mathrm{MHz}$, DMSO

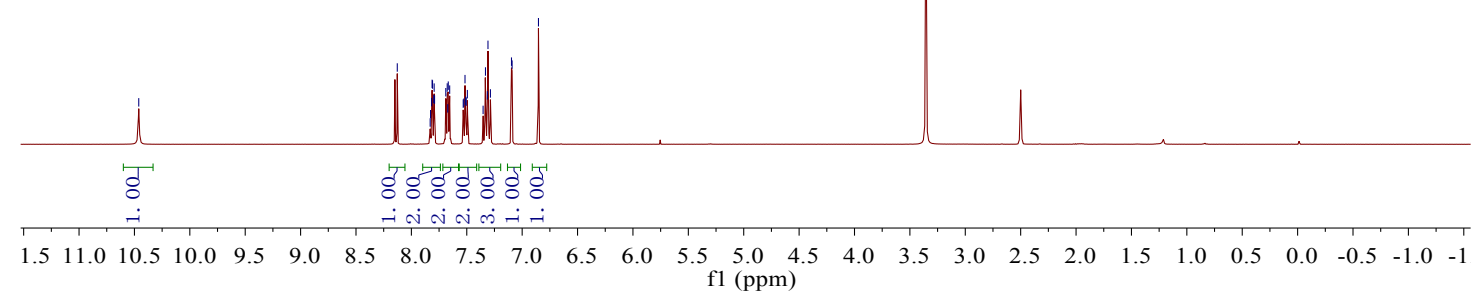

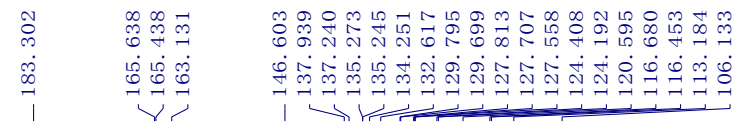<smiles>O=C1/C(=C/c2ccc(Cl)cc2NS(=O)(=O)c2ccc(F)cc2)Oc2ccccc21</smiles>

5d, ${ }^{13} \mathrm{C}$ NMR $100 \mathrm{MHz}$, DMSO

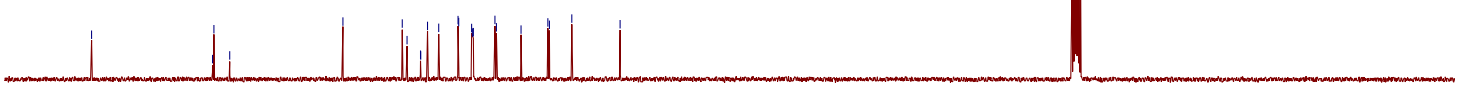

$\begin{array}{lllllllllllllllllllll}190 & 180 & 170 & 160 & 150 & 140 & 130 & 120 & 110 & 100 & 90 & 80 & 70 & 60 & 50 & 40 & 30 & 20 & 10 & 0 & -10\end{array}$ 


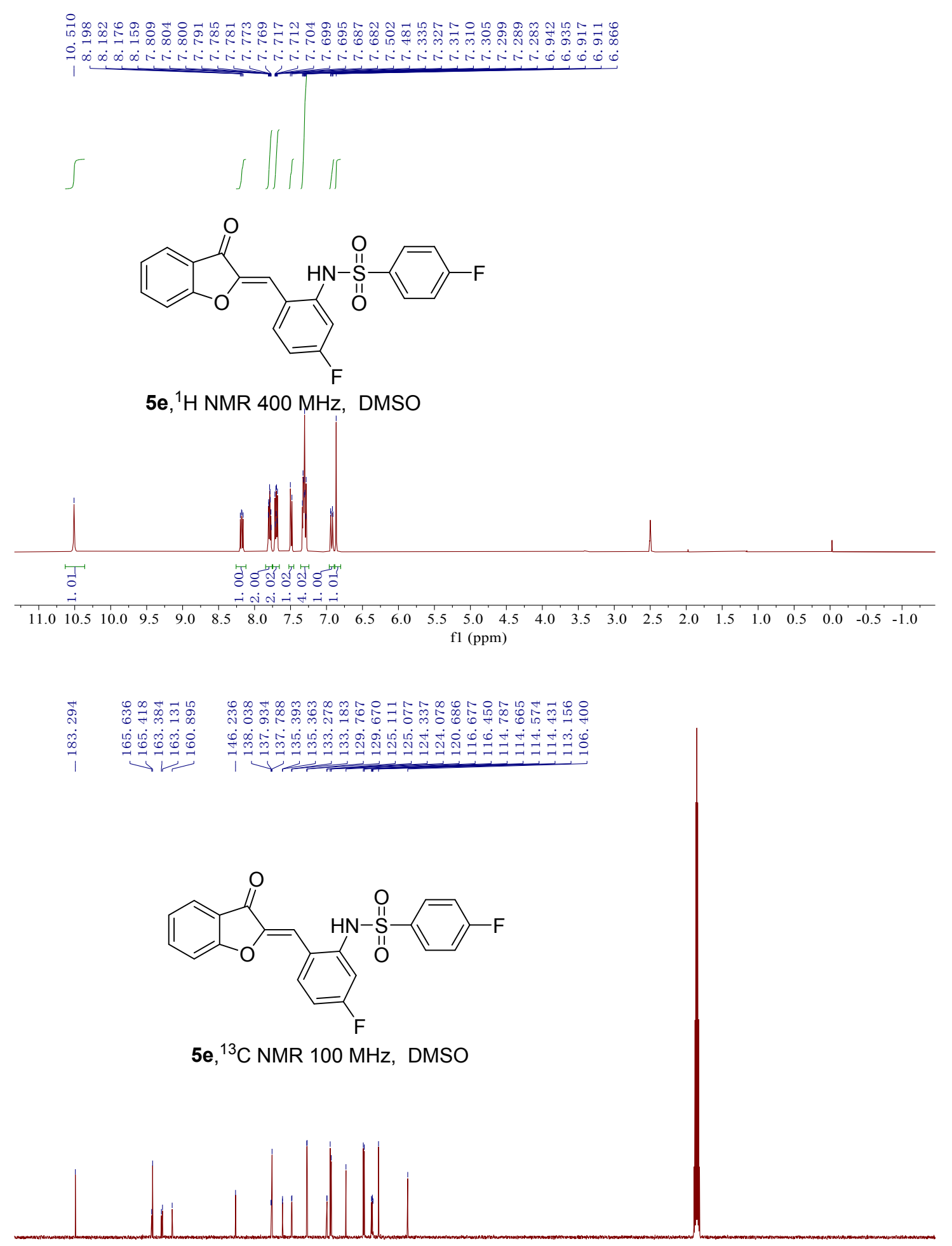

$\begin{array}{lllllllllllllllllllll}190 & 180 & 170 & 160 & 150 & 140 & 130 & 120 & 110 & 100 & 90 & 80 & 70 & 60 & 50 & 40 & 30 & 20 & 10 & 0 & -10\end{array}$ 


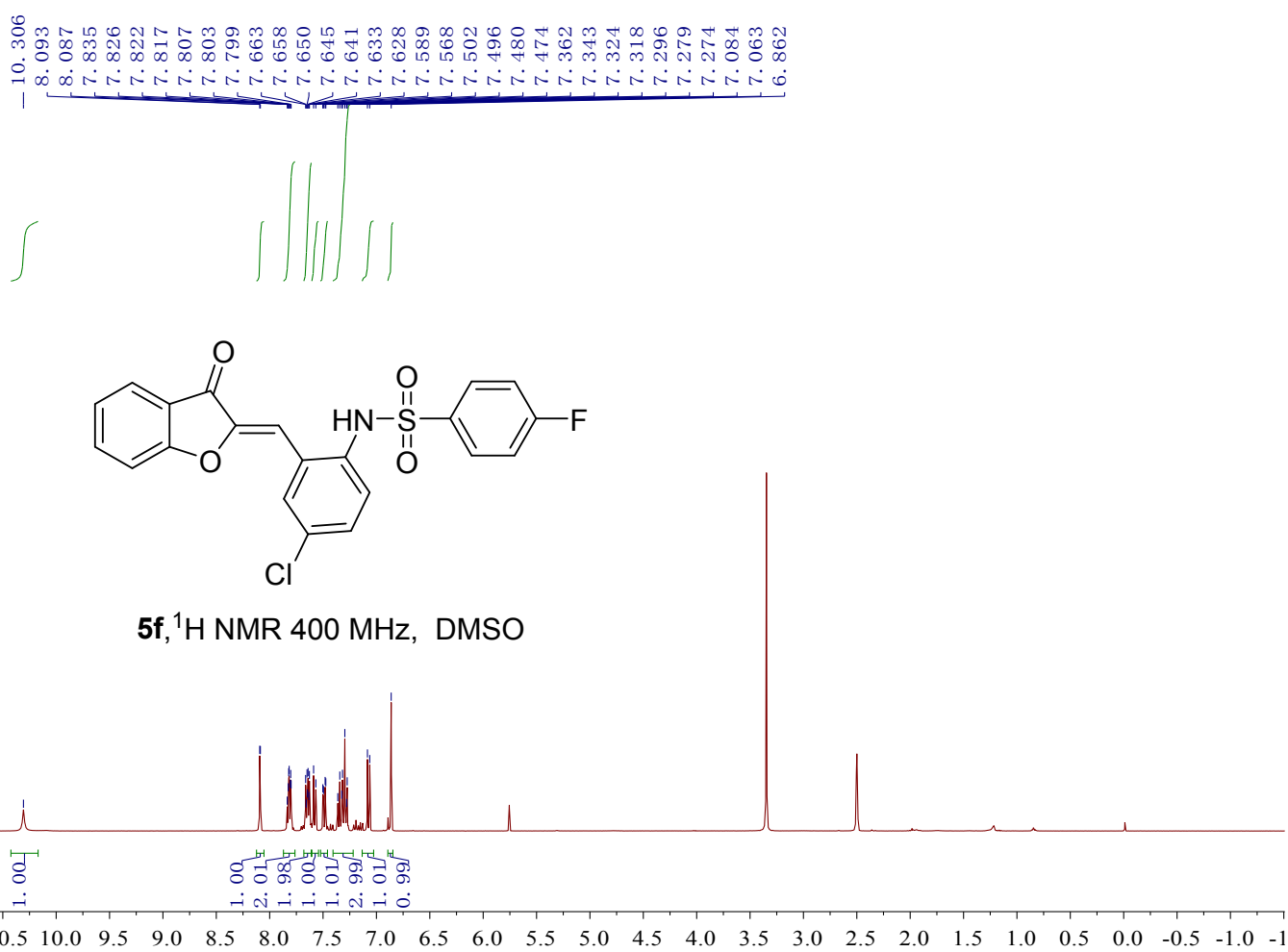

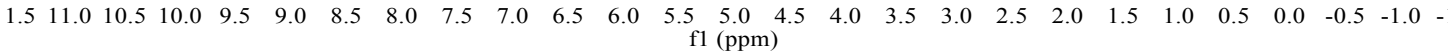
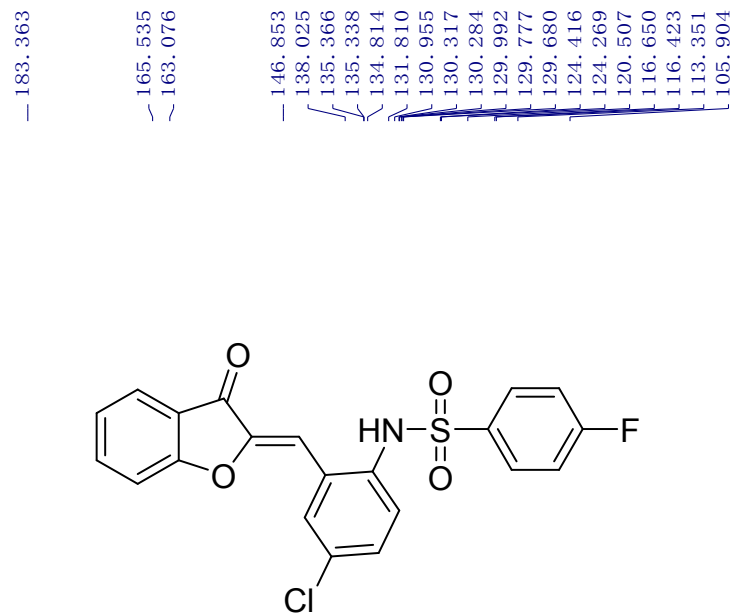

5f, ${ }^{13} \mathrm{C}$ NMR $100 \mathrm{MHz}$, DMSO

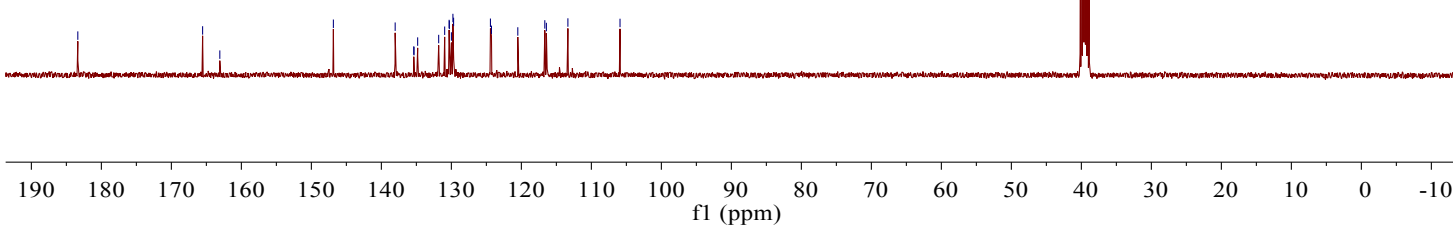



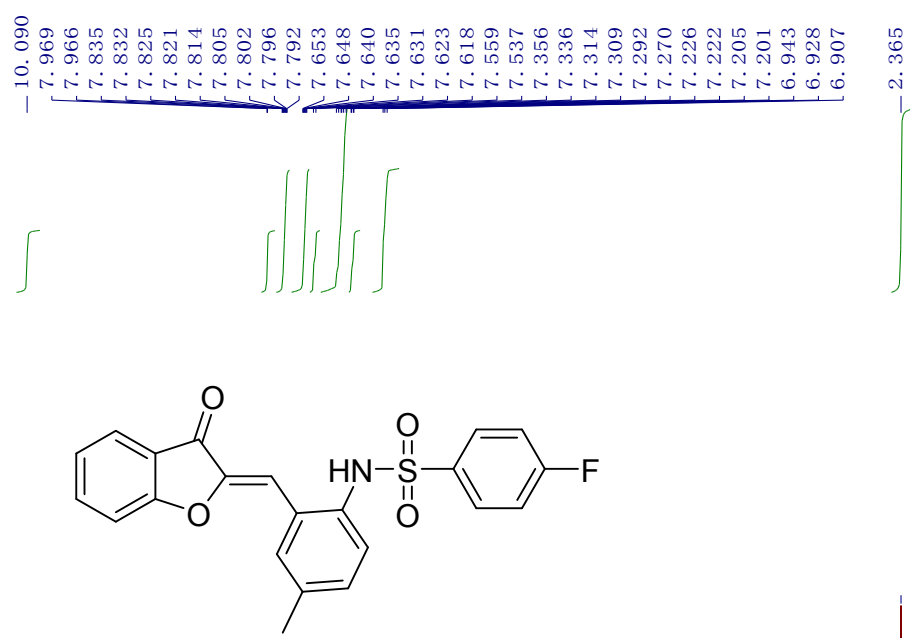

$\mathbf{5 g},{ }^{1} \mathrm{H}$ NMR $400 \mathrm{MHz}$, DMSO

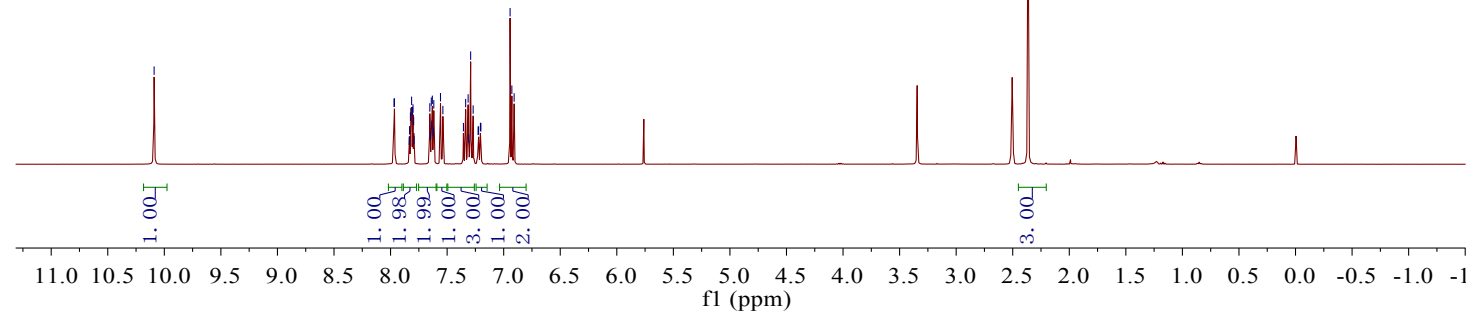

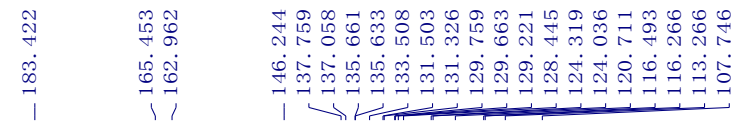

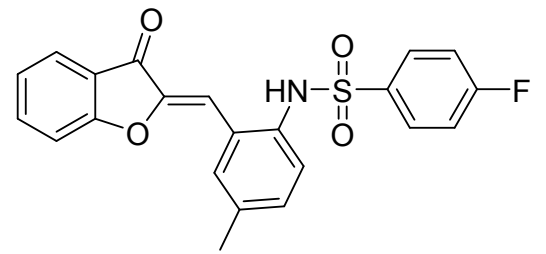

5g, ${ }^{13} \mathrm{C}$ NMR $100 \mathrm{MHz}$, DMSO

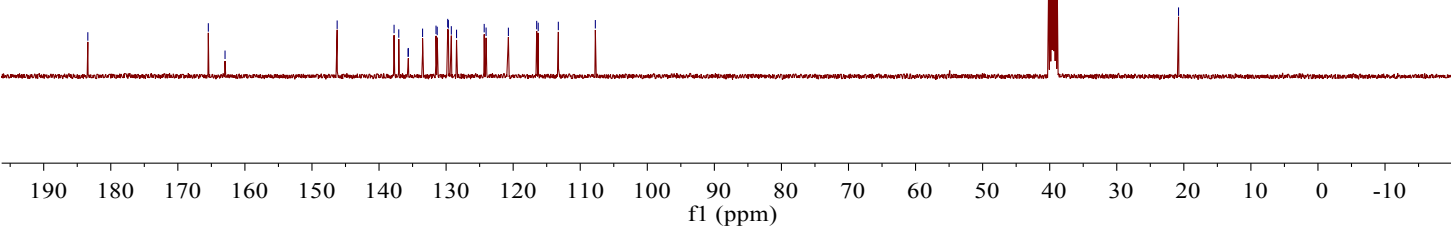




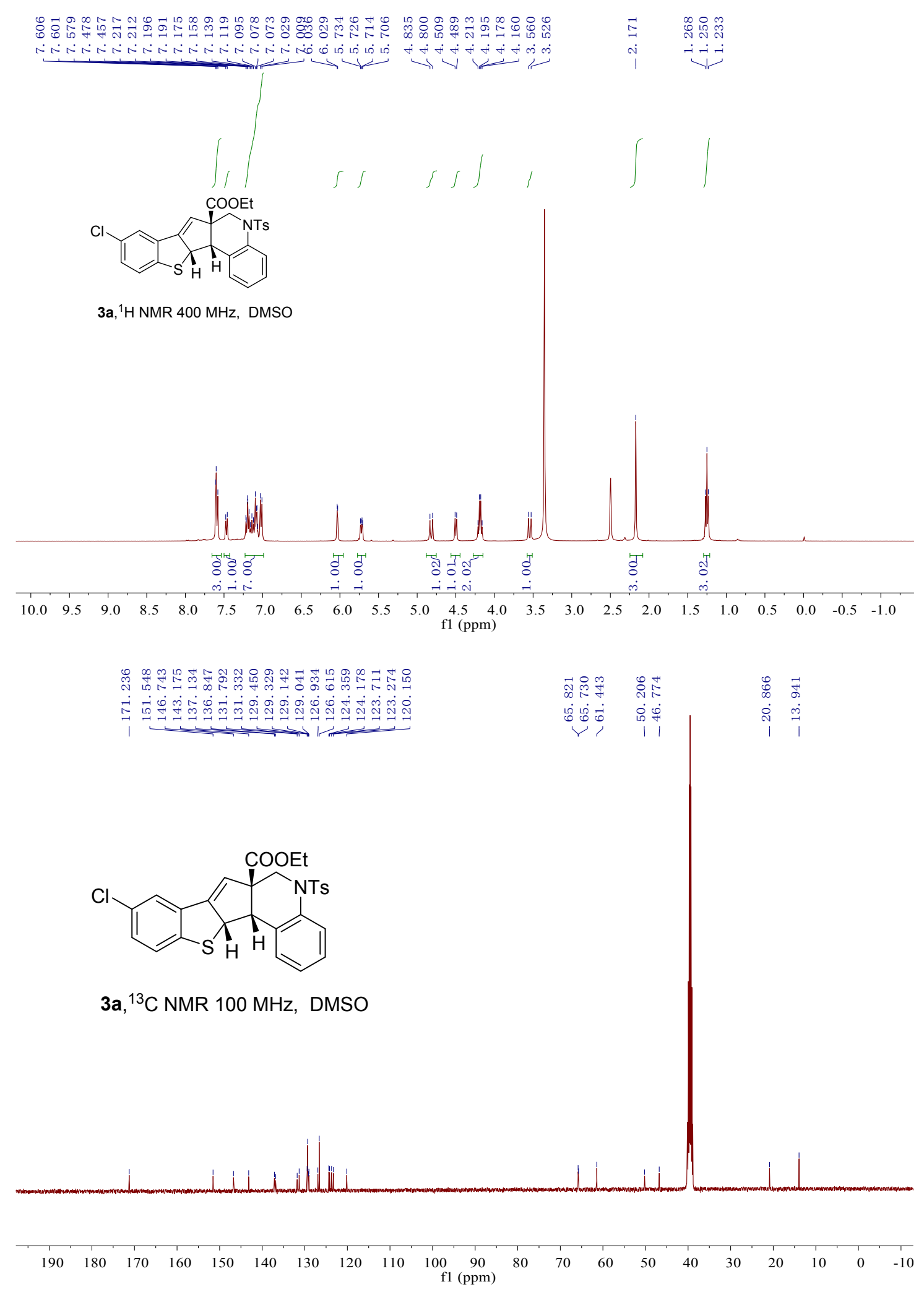


<smiles>CCOC(=O)C12C=C3c4cc(Cl)ccc4SC3C1c1ccccc1[N+]2=S</smiles>

4a, ${ }^{1} \mathrm{H}$ NMR $400 \mathrm{MHz}$, DMSO

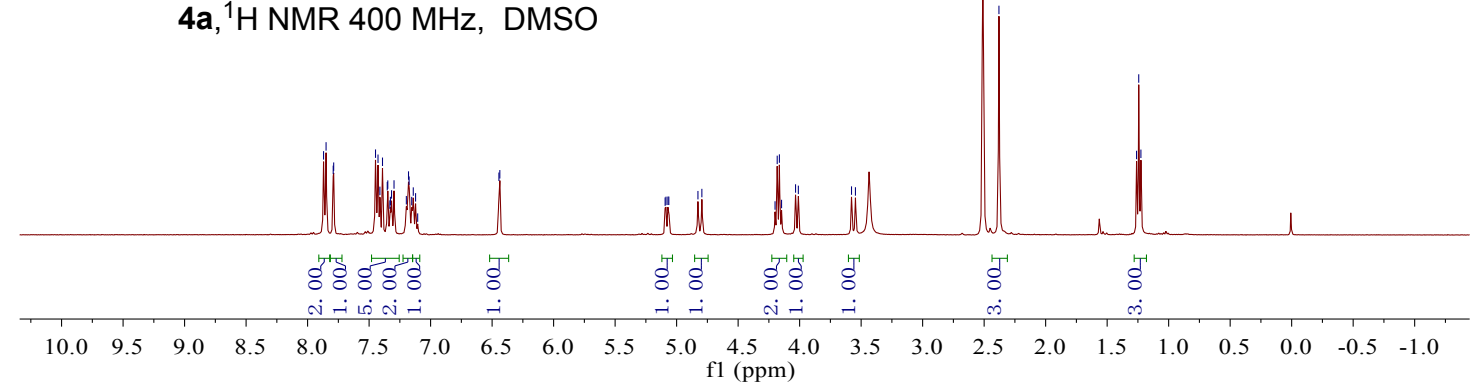

品

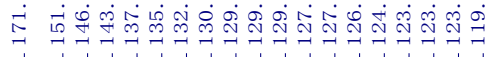<smiles>CCOC(=O)C12C=C3c4cc(Cl)ccc4S[C@H]3[C@@H]1c1ccccc1[N+]2([S-])CO</smiles>

4a, ${ }^{13} \mathrm{C}$ NMR $100 \mathrm{MHz}$, DMSO

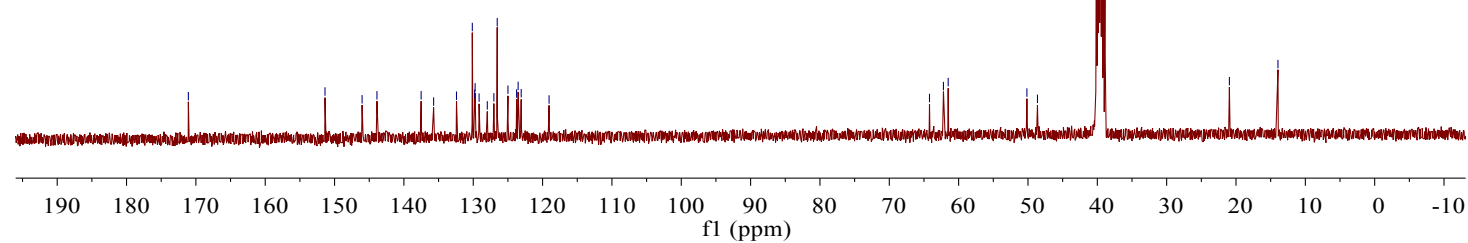




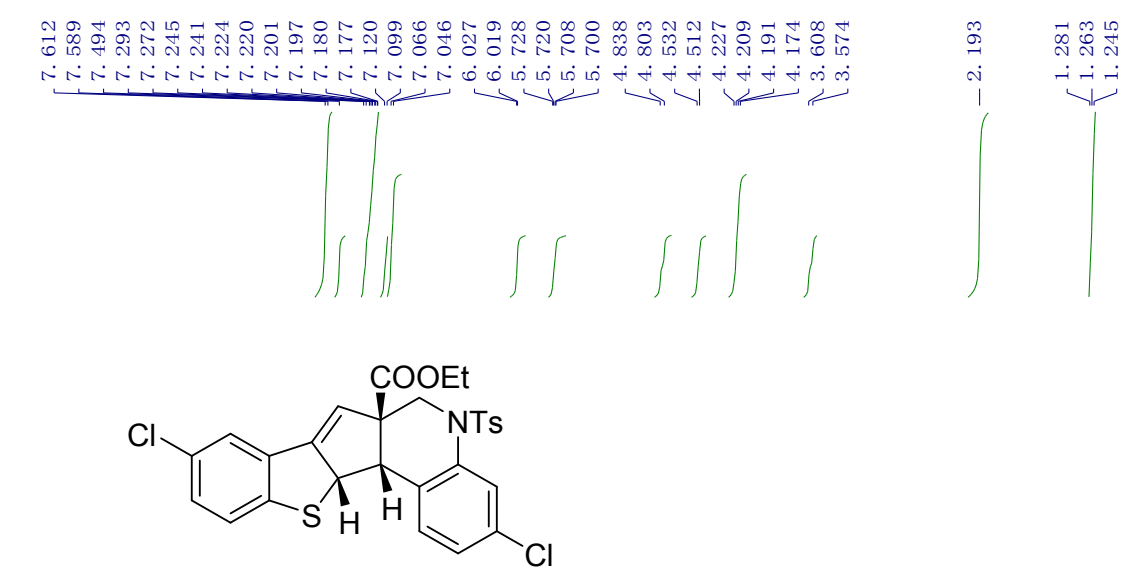

3b, ${ }^{1} \mathrm{H}$ NMR $400 \mathrm{MHz}$, DMSO
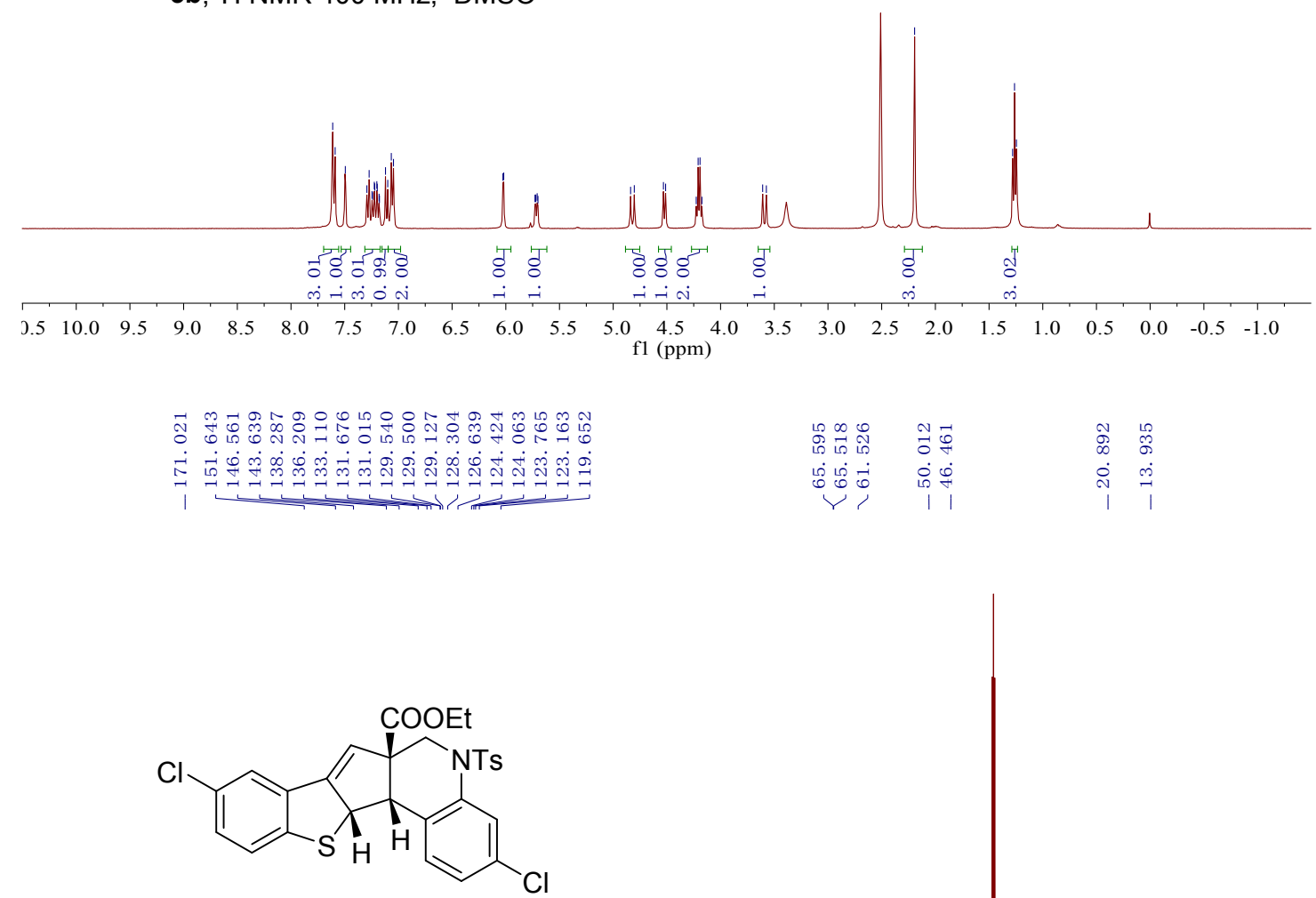

3b, ${ }^{13} \mathrm{C}$ NMR $100 \mathrm{MHz}$, DMSO

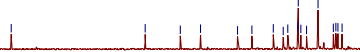

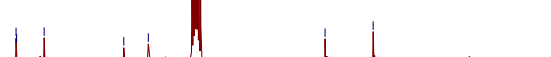

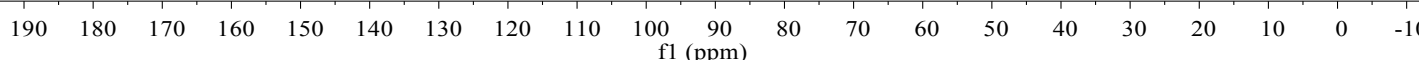




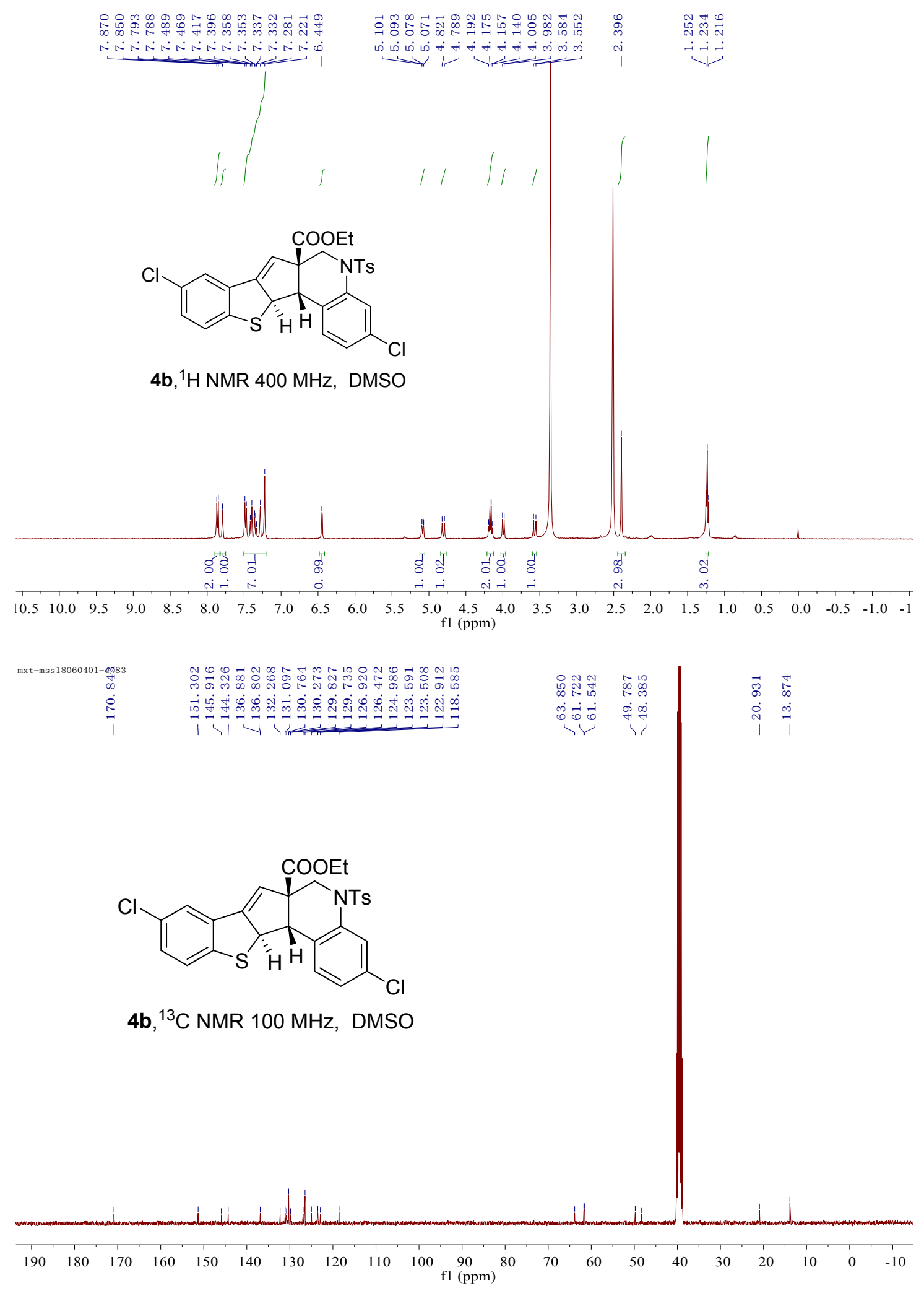


<smiles>CCOC(=O)C1(C)C=C2c3cc(Cl)ccc3S[C@H]2[C@H]2c3ccc(F)cc3[NH2+][C@@H]21</smiles>

3c, ${ }^{1} \mathrm{H}$ NMR $400 \mathrm{MHz}$, DMSO

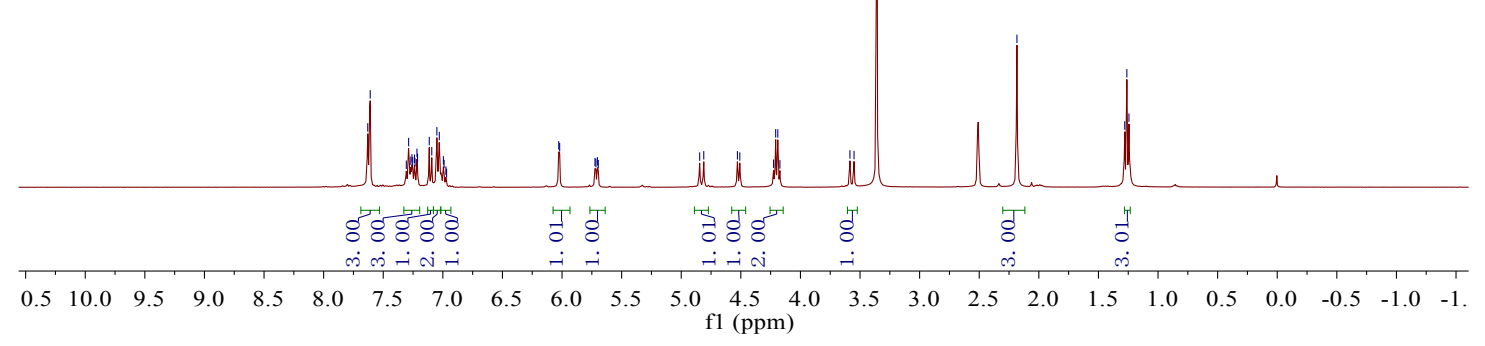

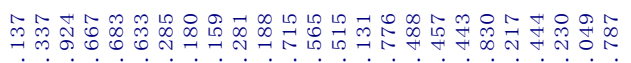

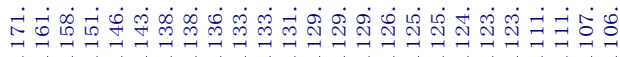

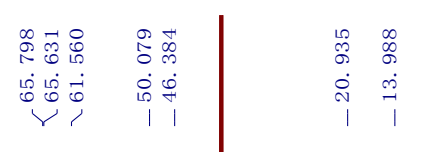<smiles>CCOC(=O)C1(C)C=C2c3cc(Cl)ccc3SC2[C@H]2c3ccc(F)cc3[NH2+][C@@H]21</smiles>

3c, ${ }^{13} \mathrm{C}$ NMR $100 \mathrm{MHz}$, DMSO

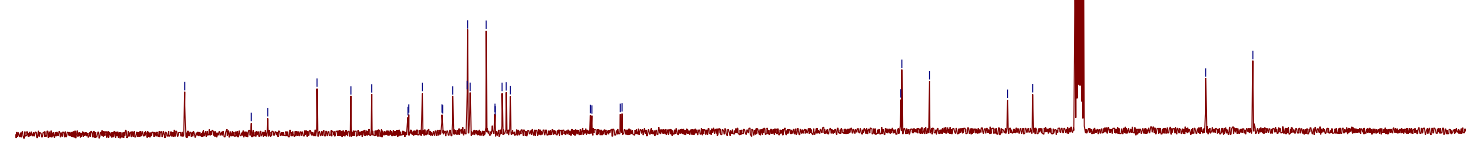

$\begin{array}{lllllllllllllllllllll}190 & 180 & 170 & 160 & 150 & 140 & 130 & 120 & 110 & 100 & \begin{array}{c}90 \\ \mathrm{f} 1(\mathrm{ppm})\end{array} & 80 & 70 & 60 & 50 & 40 & 30 & 20 & 10 & 0 & -10\end{array}$ 


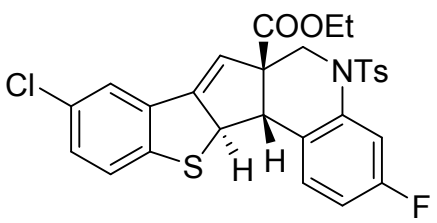

4c, ${ }^{1} \mathrm{H}$ NMR $400 \mathrm{MHz}$, DMSO

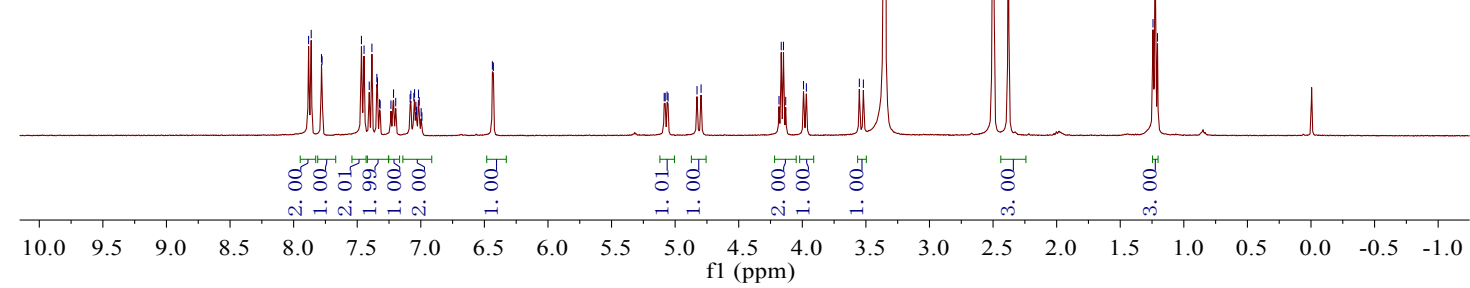

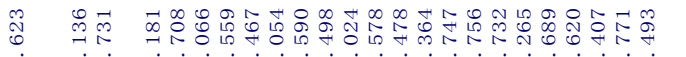

它 它点

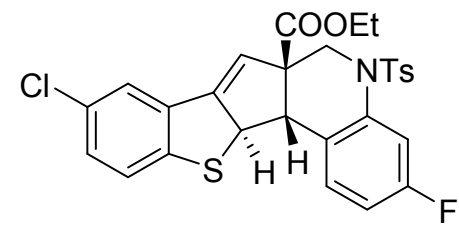

4c, ${ }^{13} \mathrm{C}$ NMR $100 \mathrm{MHz}$, DMSO

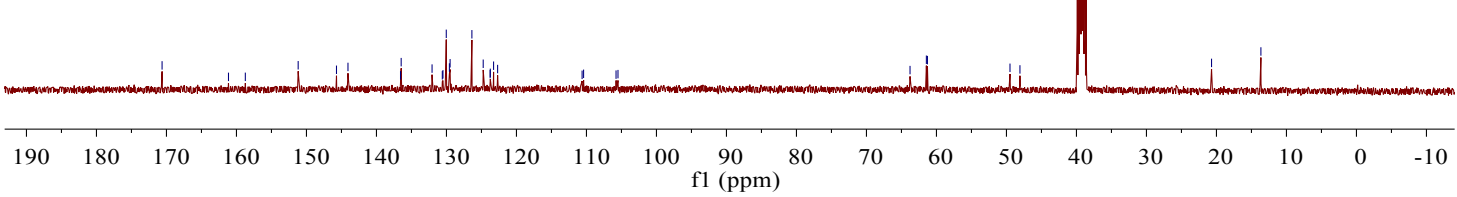




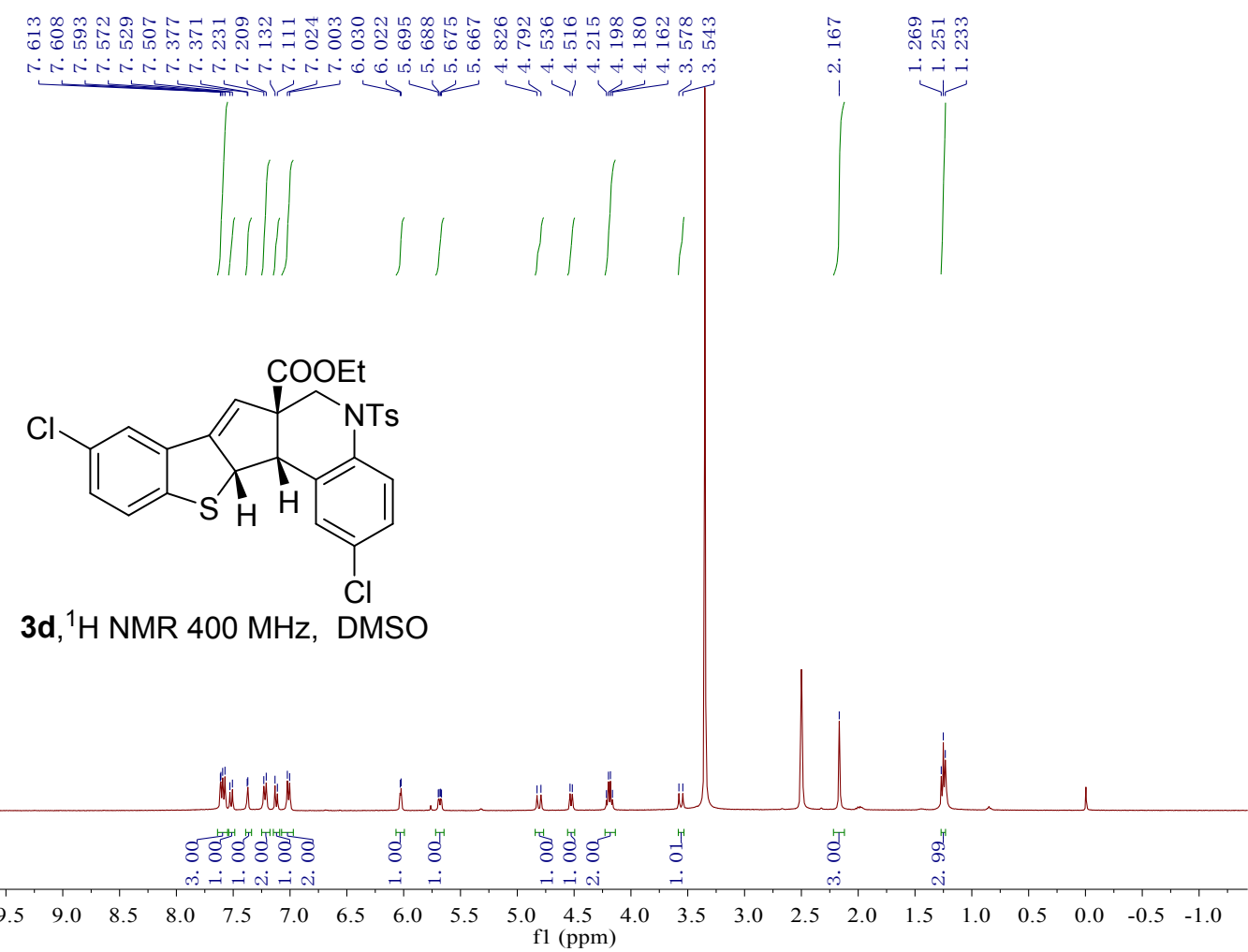

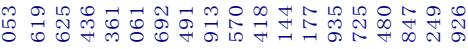

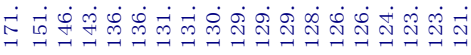

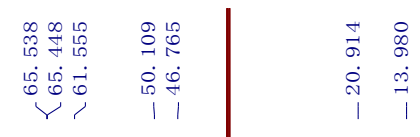<smiles>CCOC(=O)N1c2ccc(Cl)cc2[C@H]2[C@@H]3Sc4ccc(Cl)cc4C3=CC21C(=O)OCC</smiles>

3d, ${ }^{13} \mathrm{C}$ NMR $100 \mathrm{MHz}$, DMSO

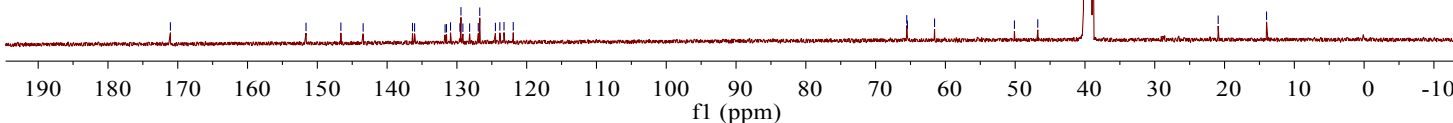



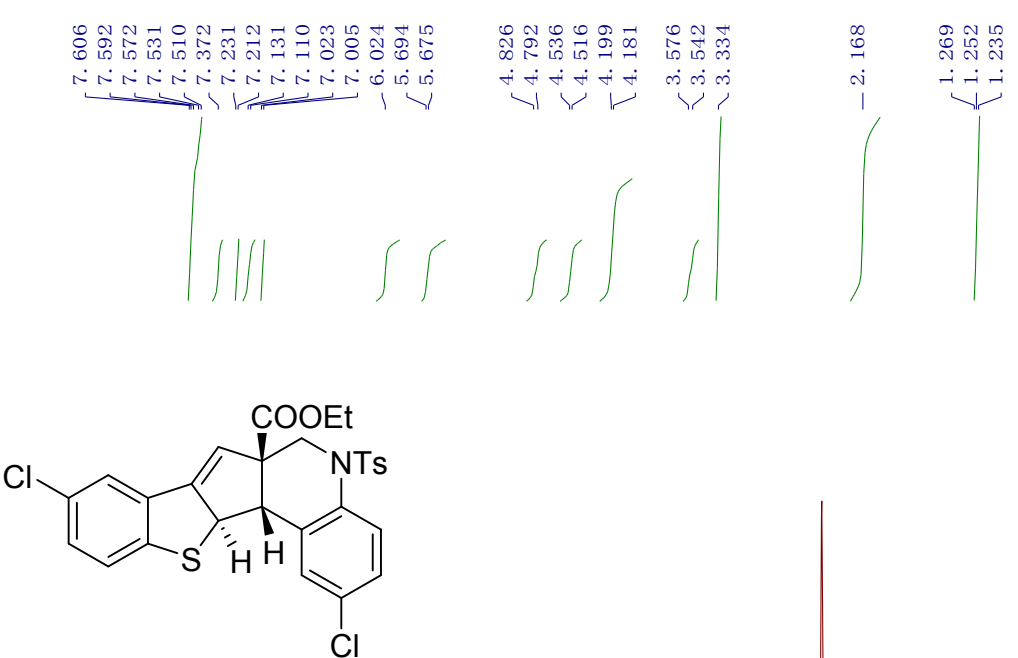

4d, ${ }^{1} \mathrm{H}$ NMR $400 \mathrm{MHz}$, DMSO

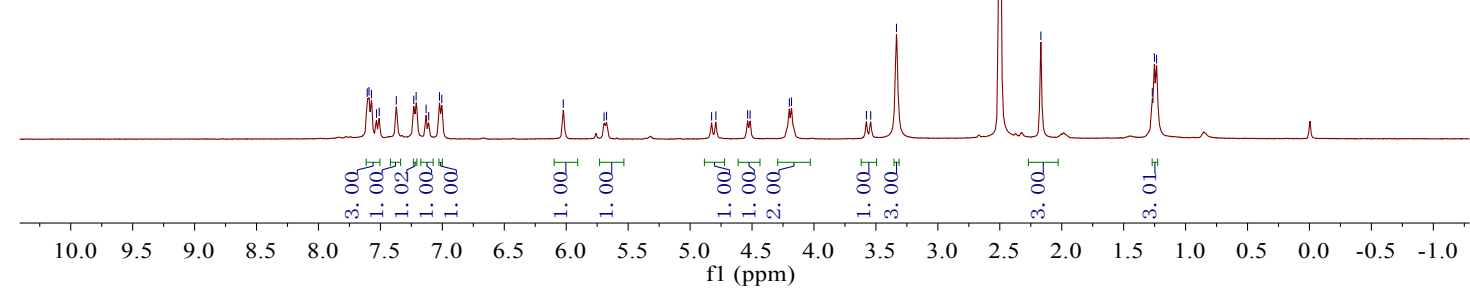

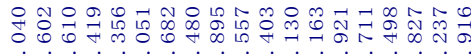

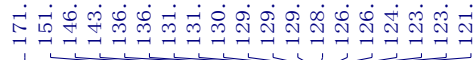

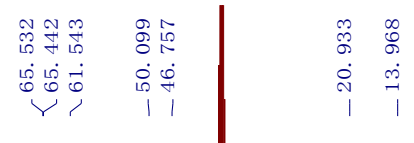<smiles>CCOC(=O)C12C=C3c4cc(Cl)ccc4SC3[C@H]1c1cc(Cl)ccc1[NH2+]C2</smiles>

4d, ${ }^{13} \mathrm{C}$ NMR $100 \mathrm{MHz}$, DMSO

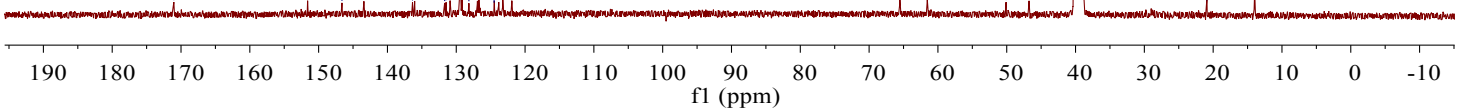




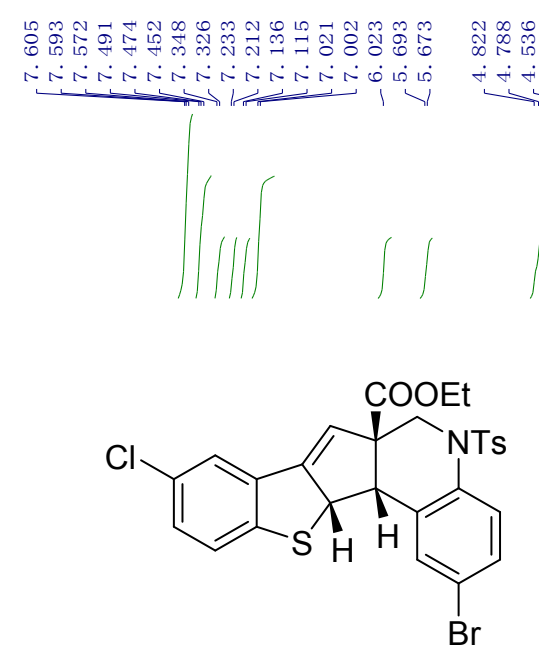

3e, ${ }^{1} \mathrm{H}$ NMR $400 \mathrm{MHz}$, DMSO

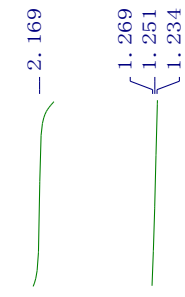

Mrict $\pi$
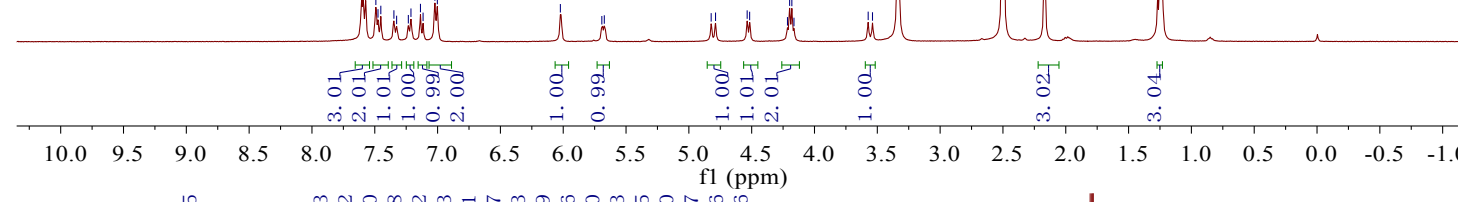

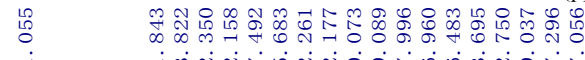

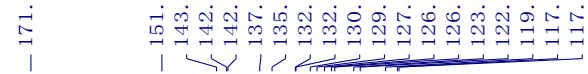

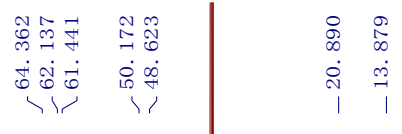<smiles>CCOC(=O)C12C=C3c4cc(Cl)ccc4S[C@H]3[C@H]1c1cc(Br)ccc1[NH2+]C2</smiles>

$3 \mathbf{e},{ }^{13} \mathrm{C}$ NMR $100 \mathrm{MHz}$, DMSO

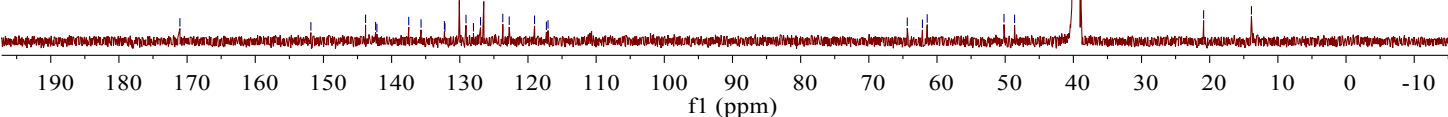




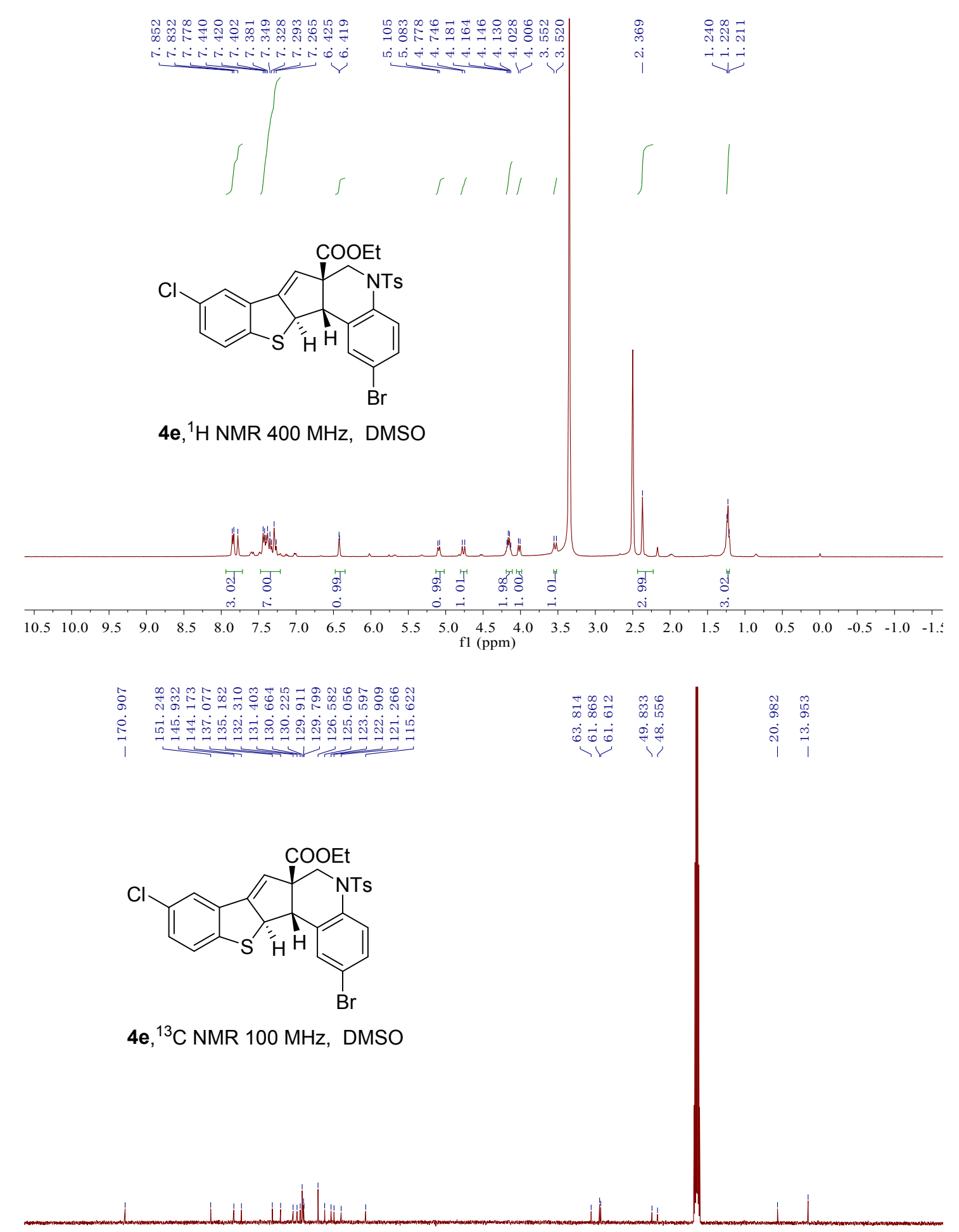

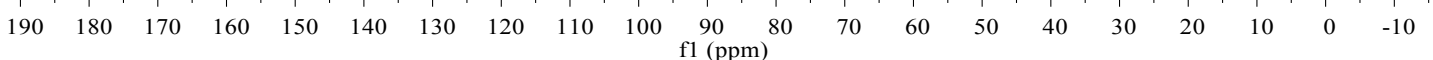




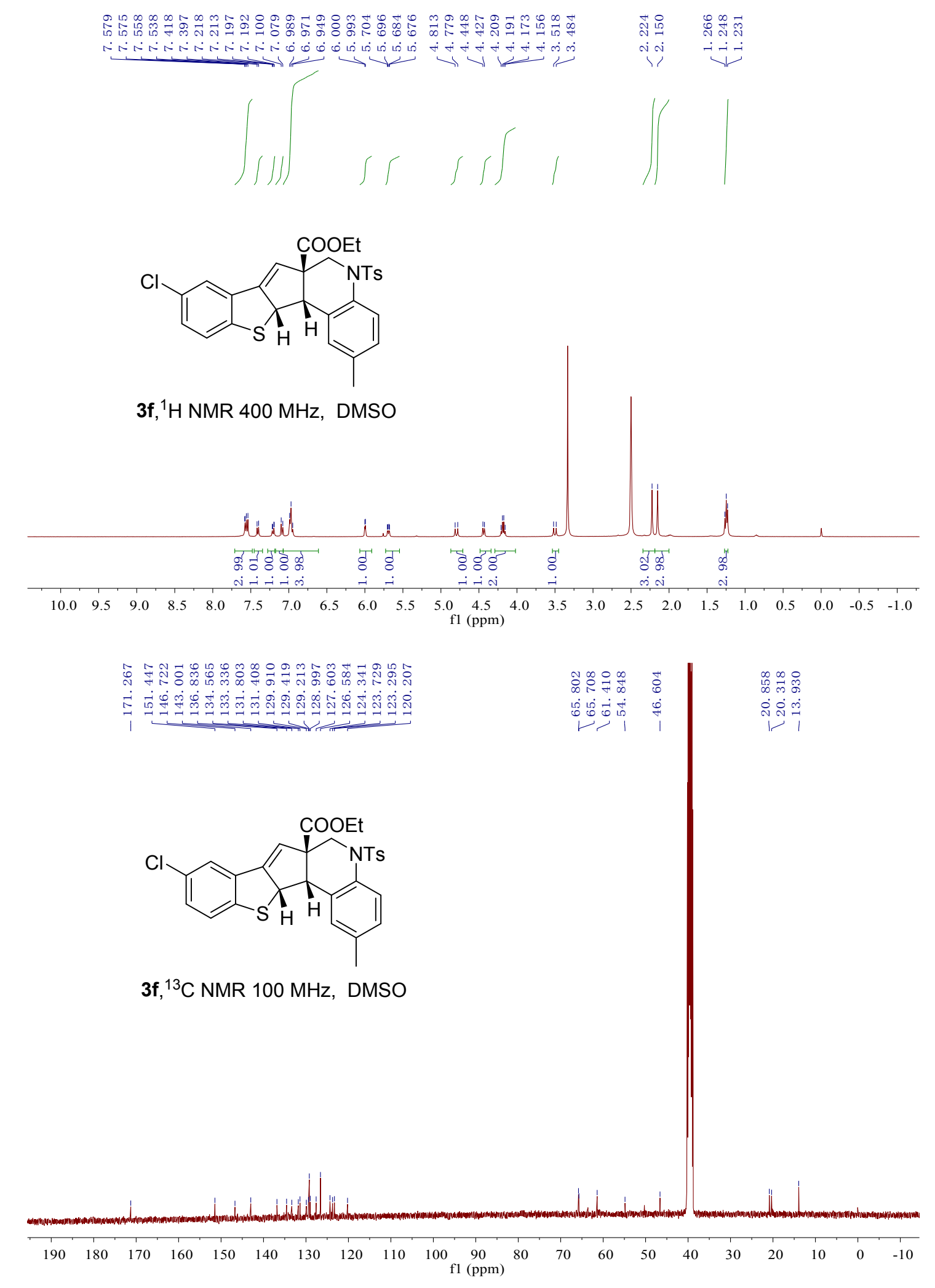




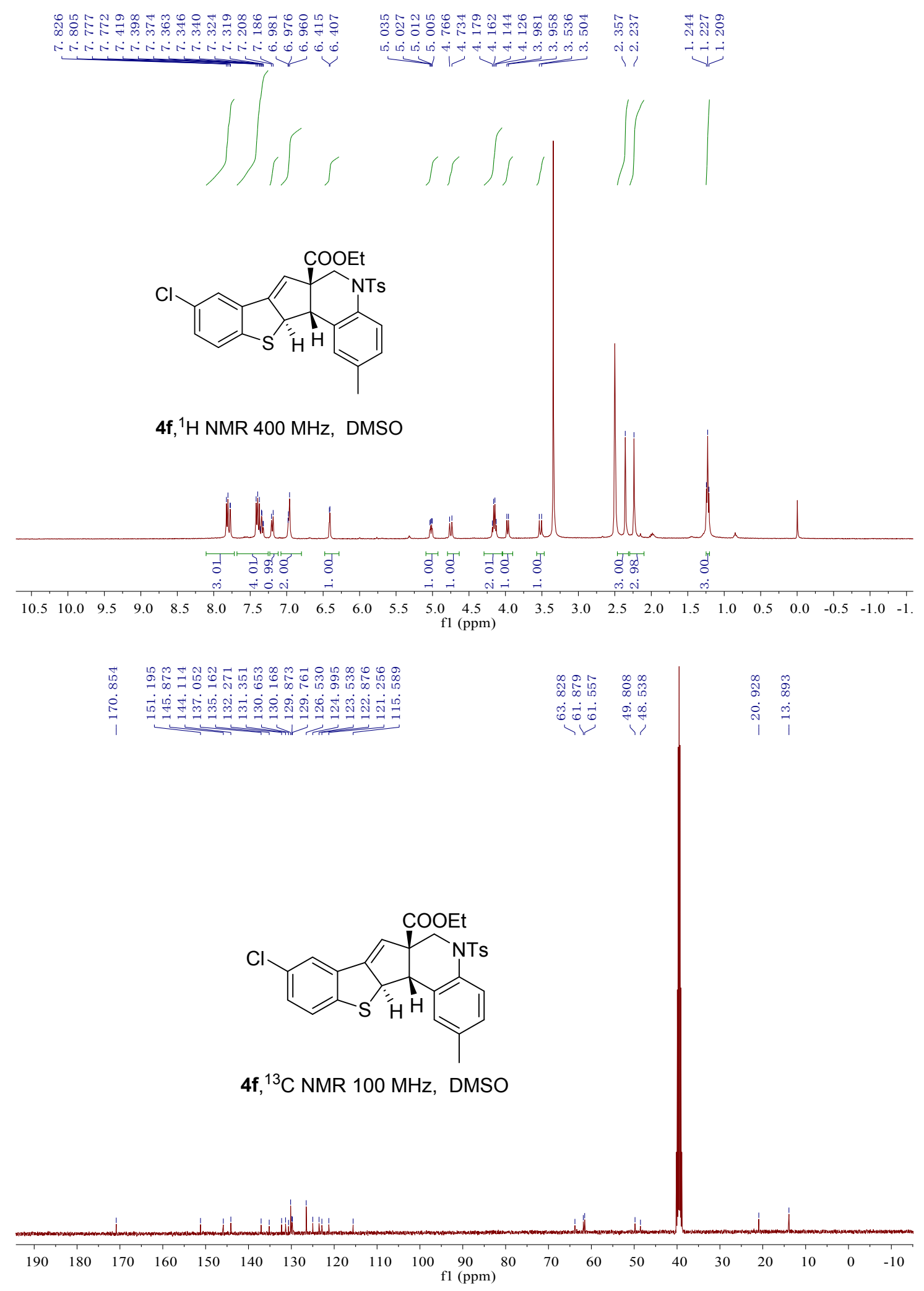


<smiles>CCOC(=O)C12C=C3c4cc(Br)ccc4S[C@H]3[C@H]1c1ccccc1[N+]2(C)[S-]</smiles>

3g, ${ }^{1} \mathrm{H}$ NMR $400 \mathrm{MHz}$, DMSO

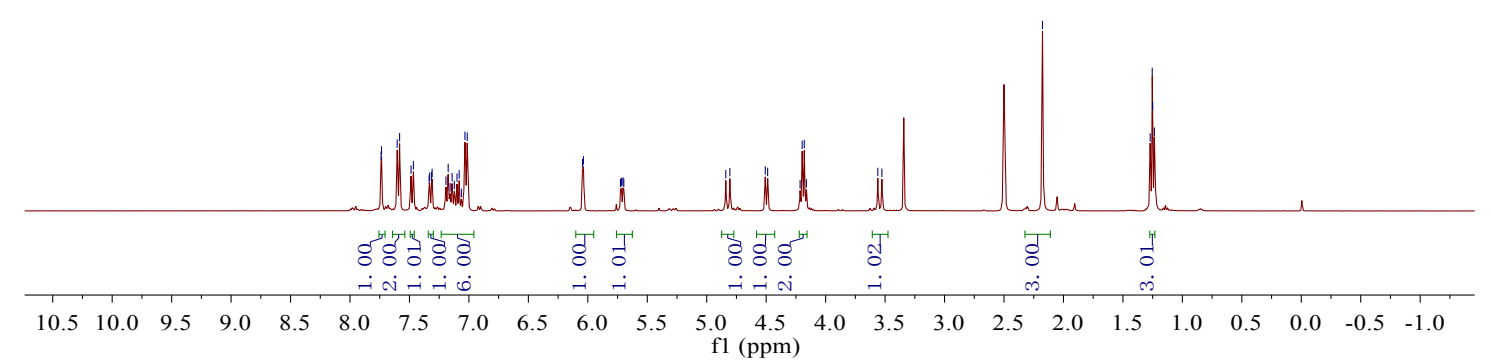

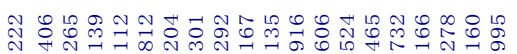

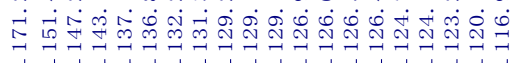<smiles>CCOC(=O)C12C=C3c4cc(Br)ccc4S[C@H]3C1c1ccccc1[N+]2(O)[S-]</smiles>

3g, ${ }^{13} \mathrm{C}$ NMR $100 \mathrm{MHz}$, DMSO

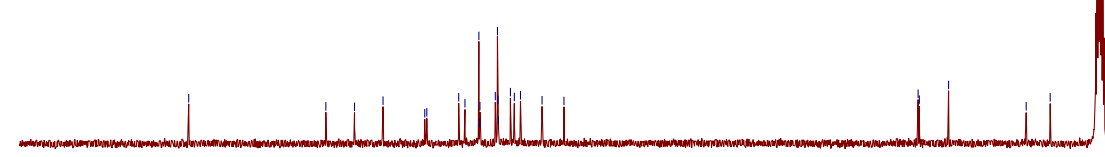


<smiles>CCOC(=O)C12C=C3c4cc(Br)ccc4SC3C1c1ccccc1[N+]2(C)[S-]</smiles>

$\mathbf{4 g},{ }^{1} \mathrm{H}$ NMR $400 \mathrm{MHz}$, DMSO

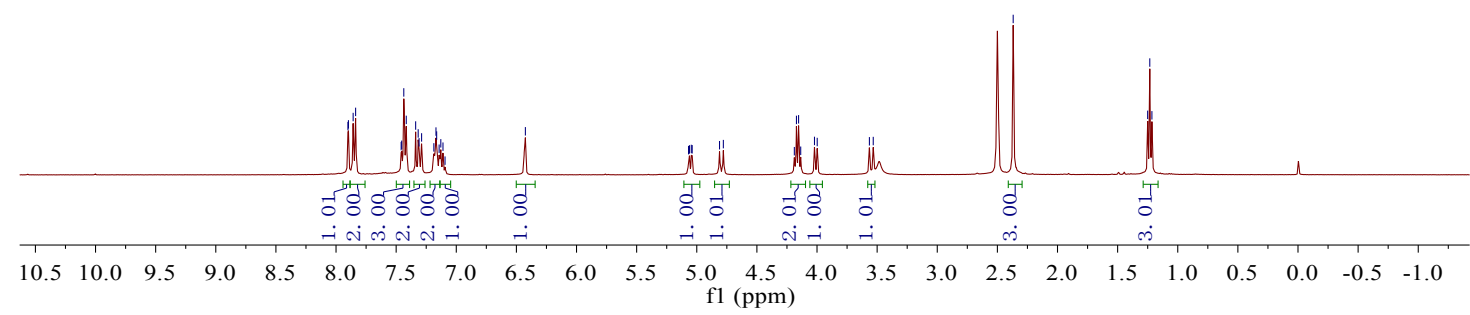

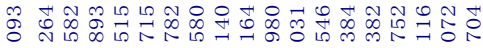

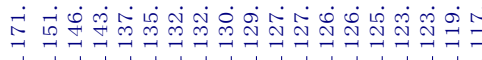

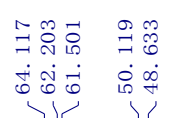

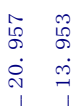<smiles>CCOC(=O)C12C=C3c4cc(Br)ccc4SC3C1c1ccccc1[N+]2=S</smiles>

$\mathbf{4 g},{ }^{1} \mathrm{H}$ NMR $400 \mathrm{MHz}$, DMSO

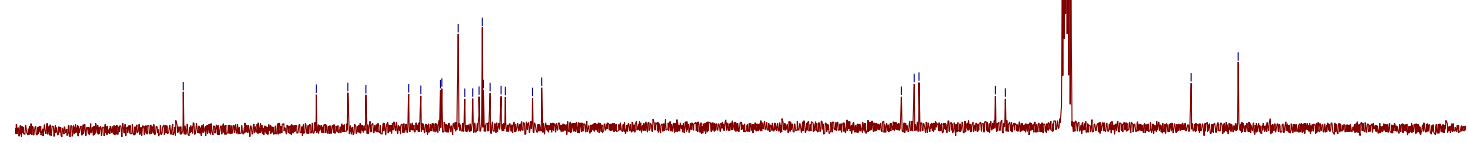

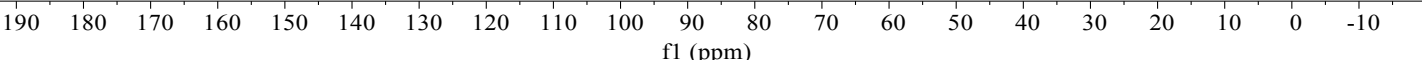




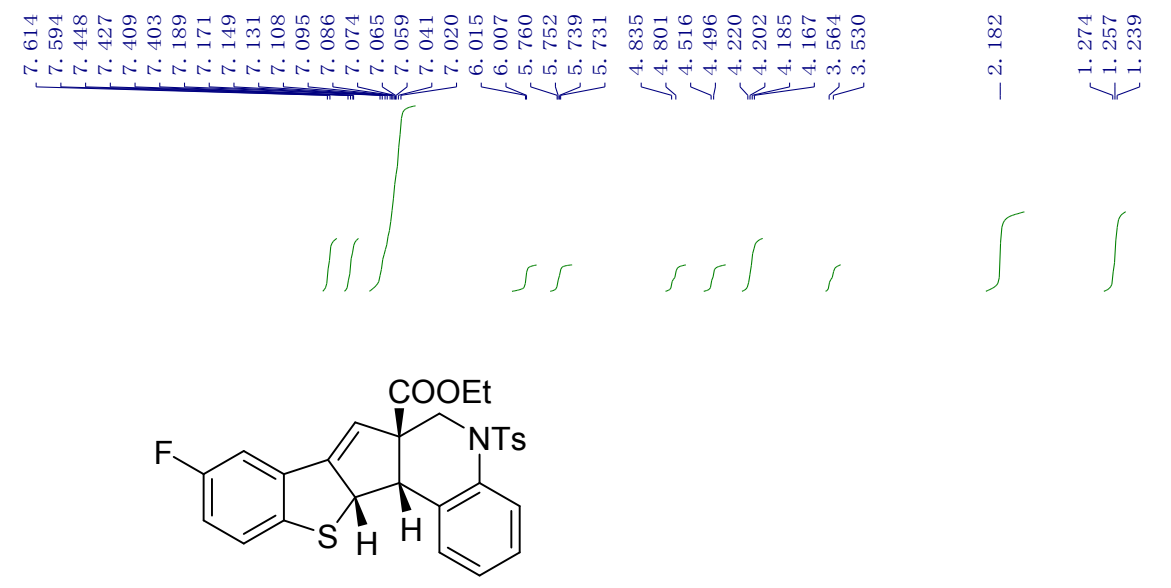

3h, ${ }^{1} \mathrm{H}$ NMR $400 \mathrm{MHz}$, DMSO
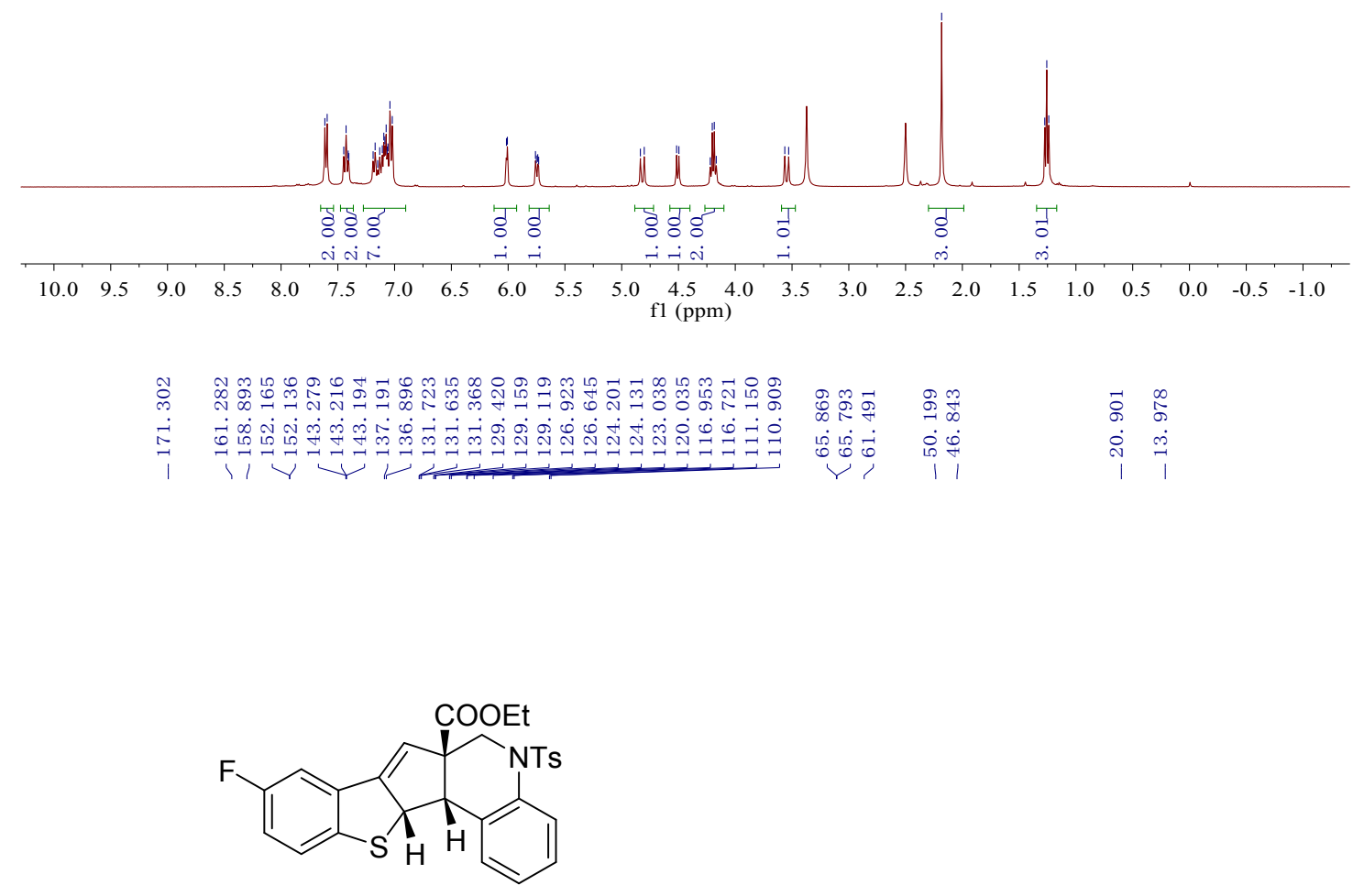

3h, ${ }^{13} \mathrm{C}$ NMR $100 \mathrm{MHz}$, DMSO

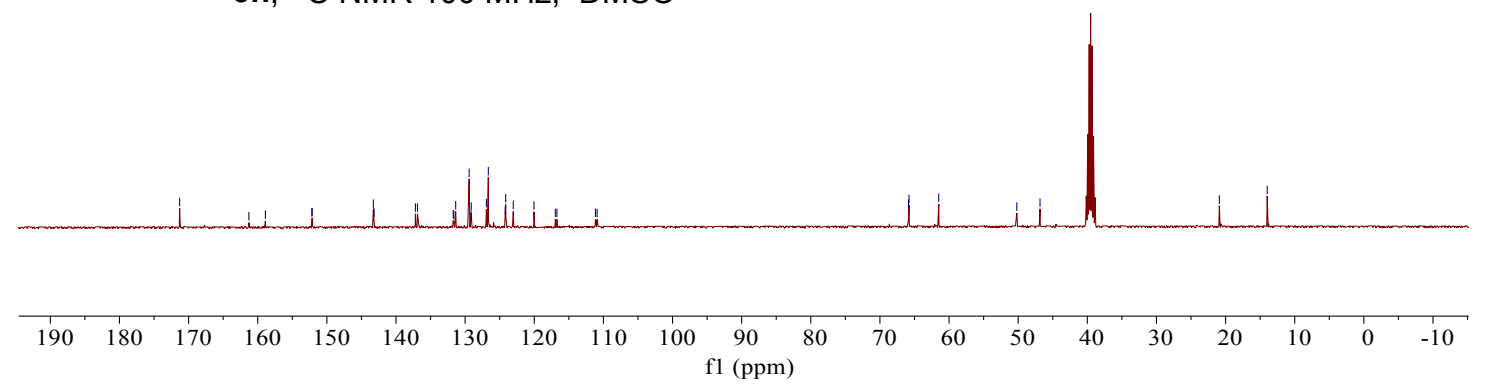




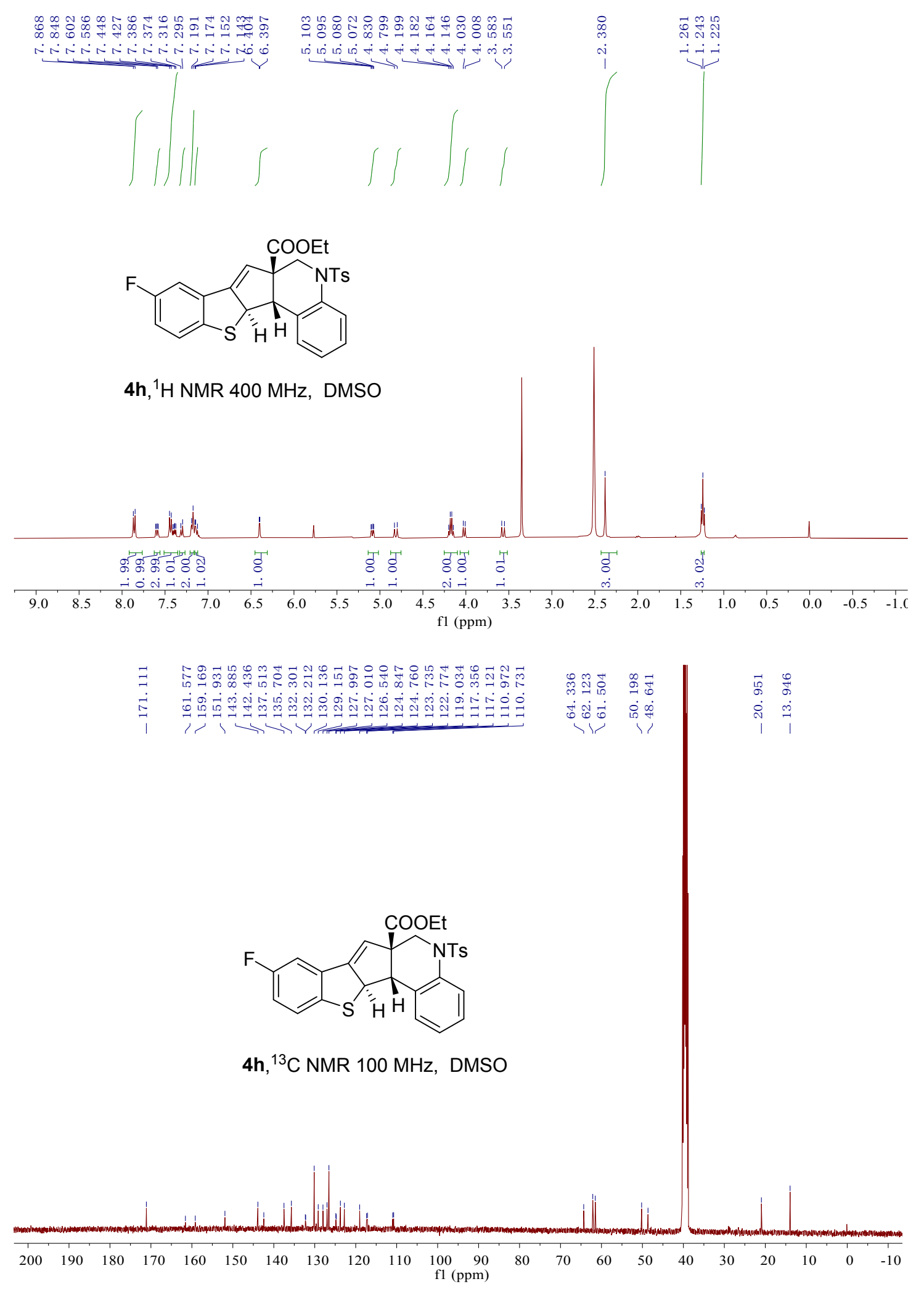



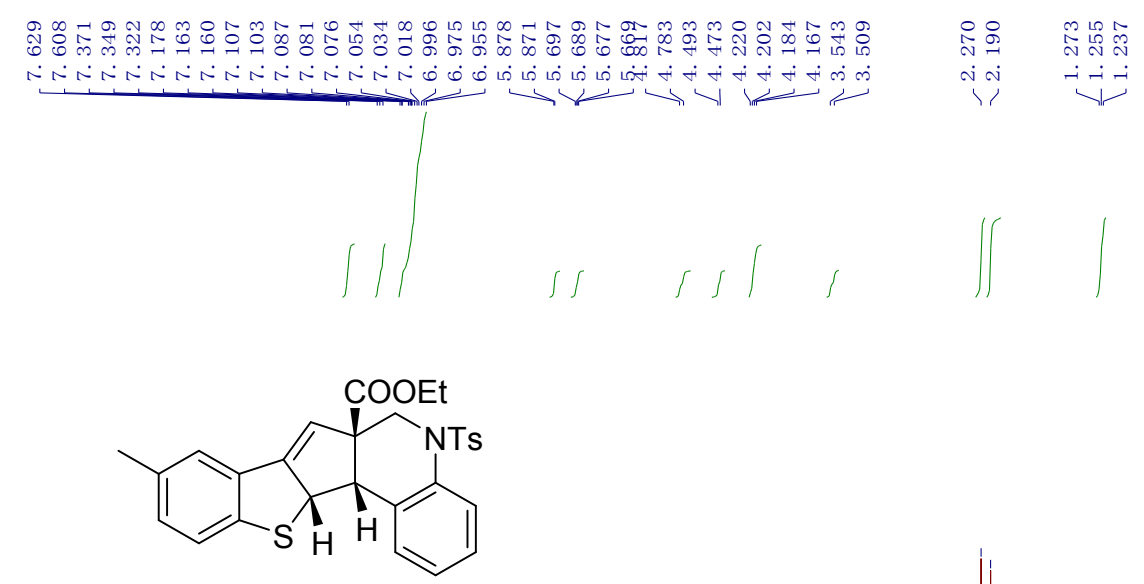

3i, ${ }^{1} \mathrm{H}$ NMR $400 \mathrm{MHz}$, DMSO

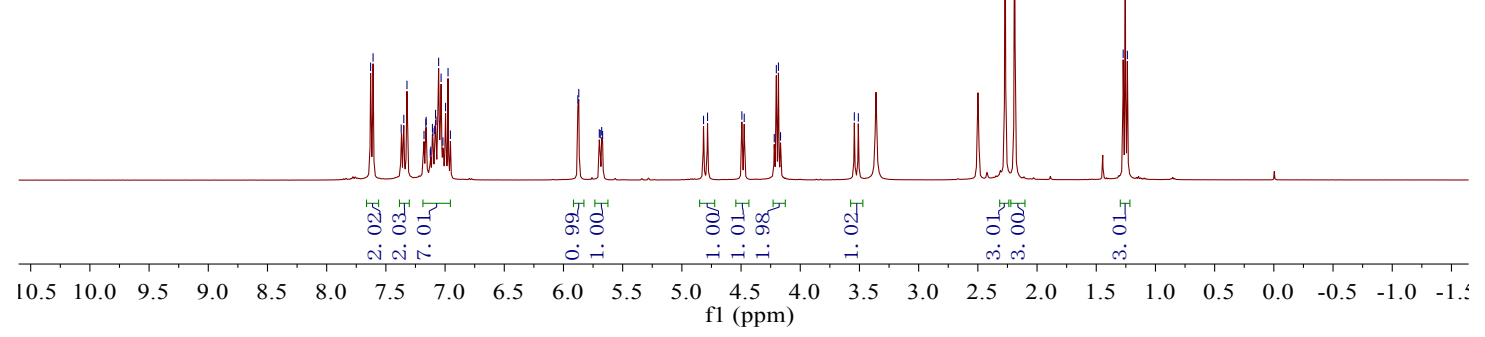

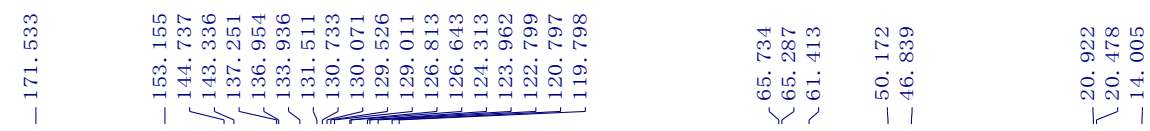

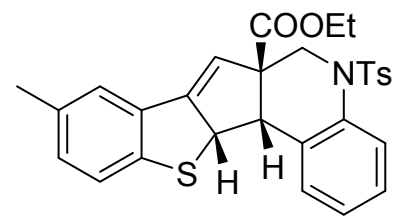

3i, ${ }^{13} \mathrm{C}$ NMR $100 \mathrm{MHz}$, DMSO

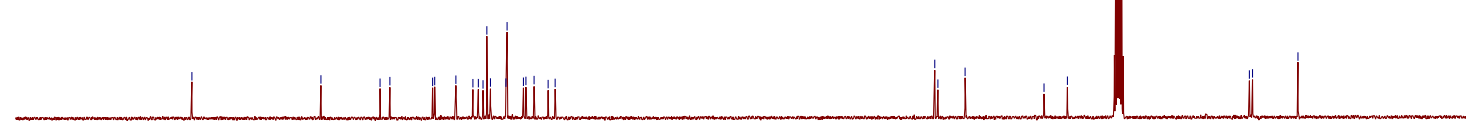

$\begin{array}{llllllllllllllllllll}190 & 180 & 170 & 160 & 150 & 140 & 130 & 120 & 110 & 100 & 90 & 80 & 70 & 60 & 50 & 40 & 30 & 20 & 10 & 0\end{array}$ 


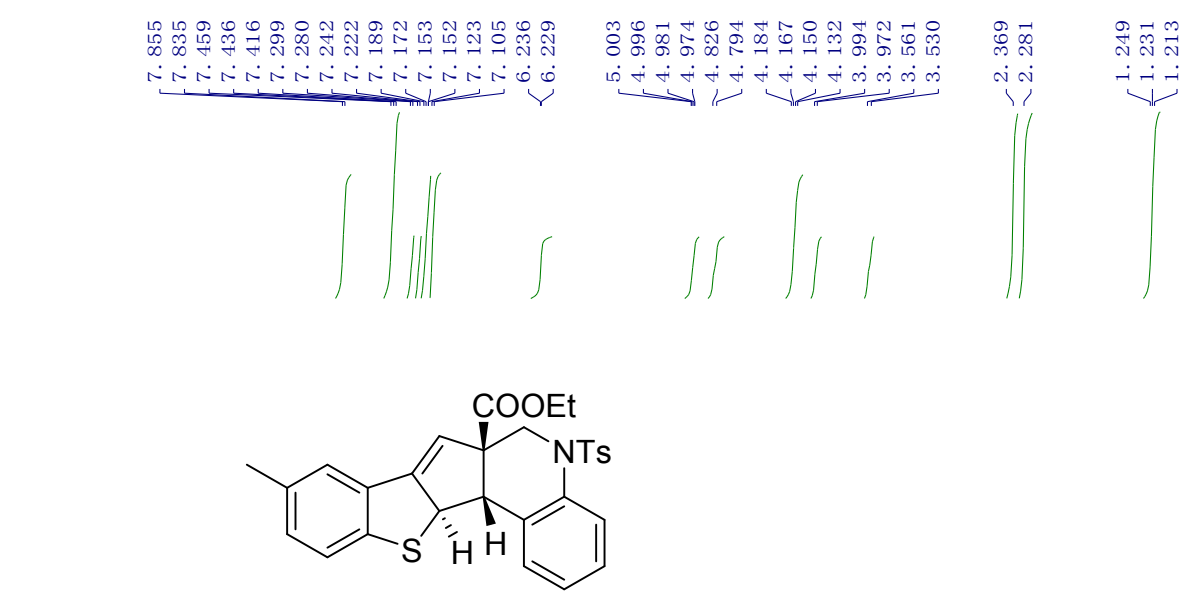

4i, ${ }^{1} \mathrm{H}$ NMR $400 \mathrm{MHz}$, DMSO
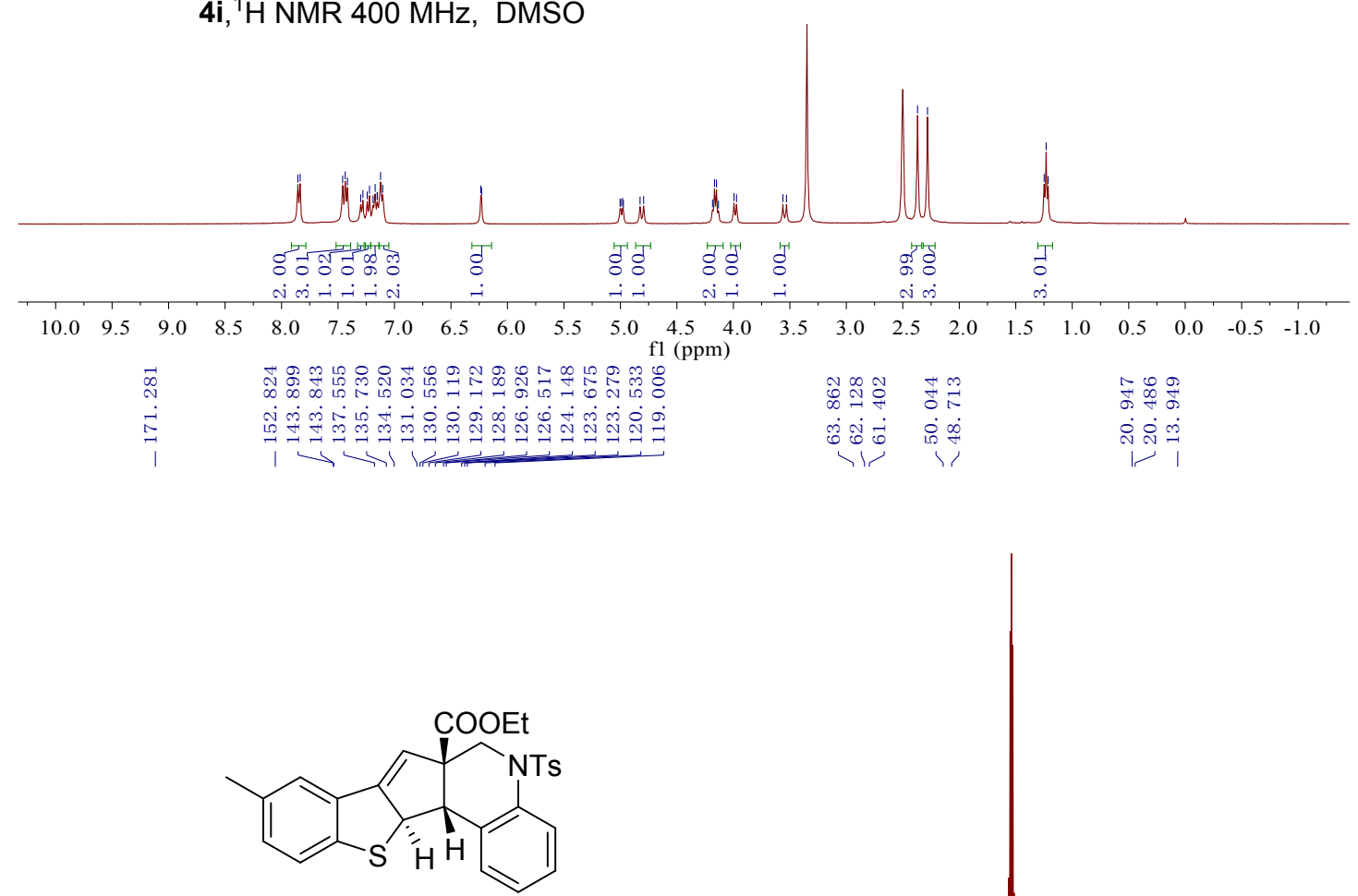

4i, ${ }^{13} \mathrm{C}$ NMR $100 \mathrm{MHz}$, DMSO

$\begin{array}{lllllllllllllllllllll}190 & 180 & 170 & 160 & 150 & 140 & 130 & 120 & 110 & 100 & \underset{\mathrm{f} 1}{90}(\mathrm{pm}) & 80 & 70 & 60 & 50 & 40 & 30 & 20 & 10 & 0 & -10\end{array}$ 

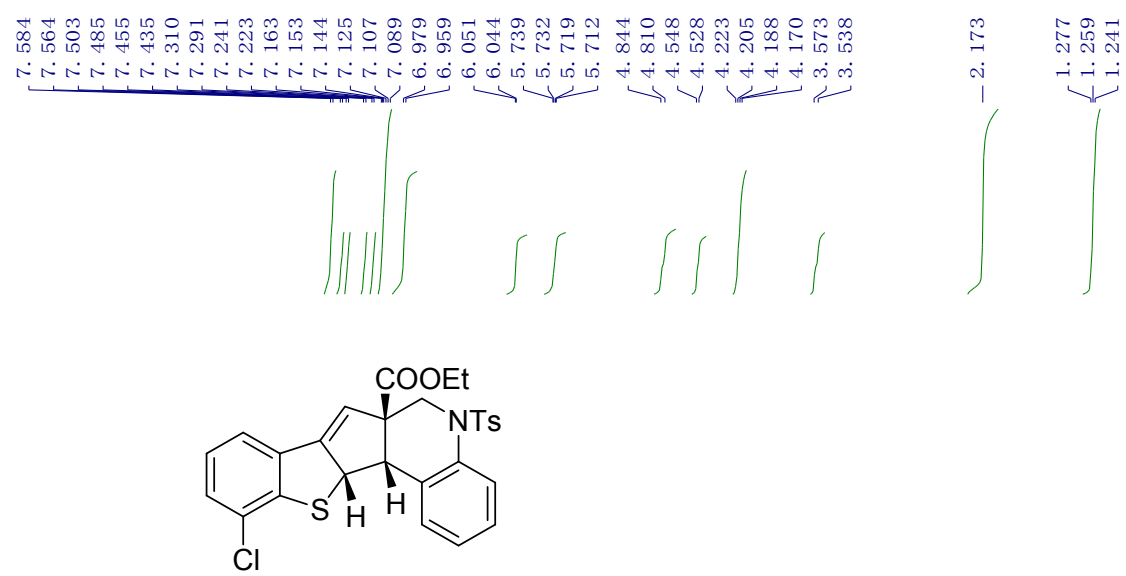

3j, ${ }^{1} \mathrm{H}$ NMR $400 \mathrm{MHz}$, DMSO
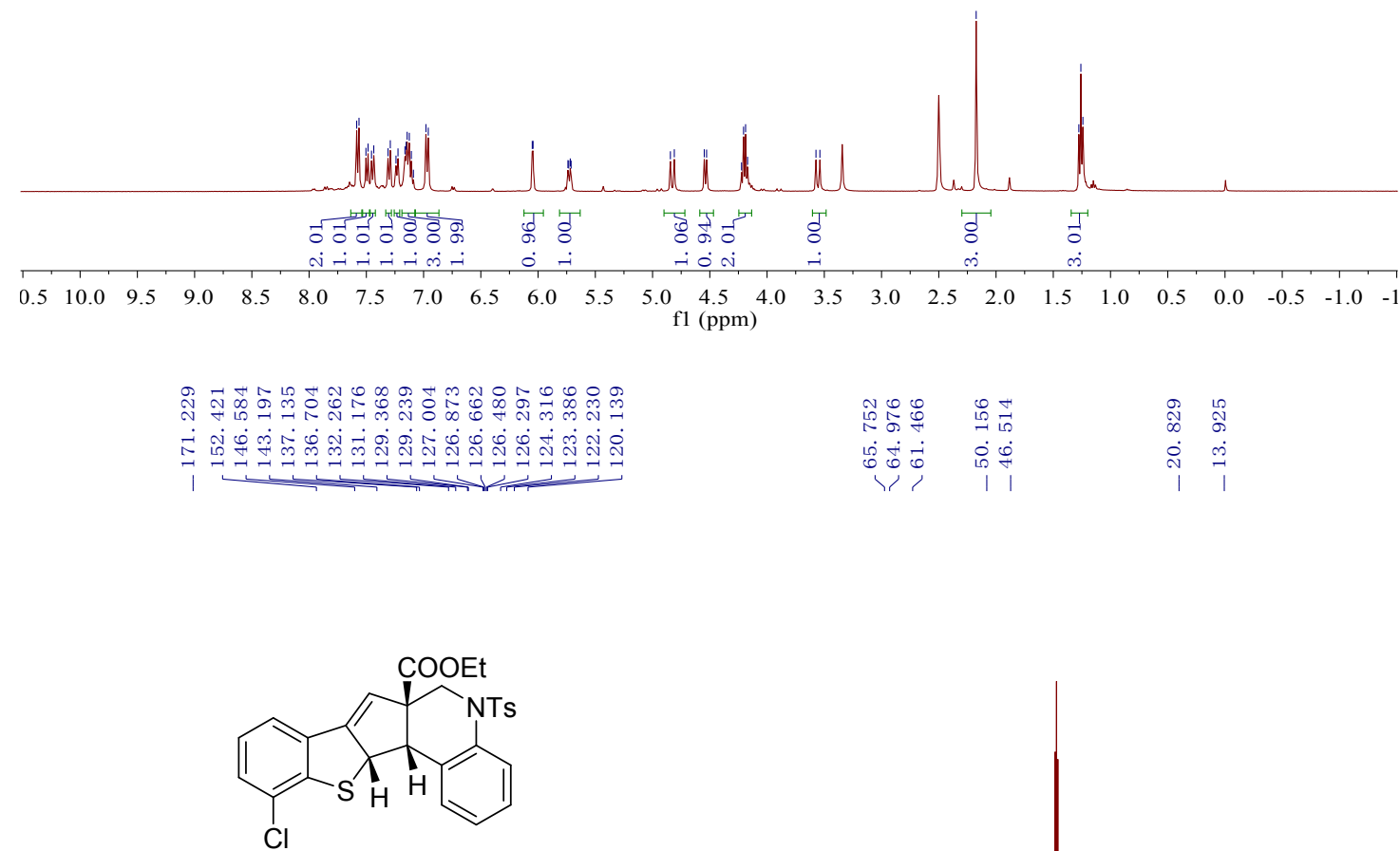

3j, ${ }^{13} \mathrm{C}$ NMR $100 \mathrm{MHz}$, DMSO

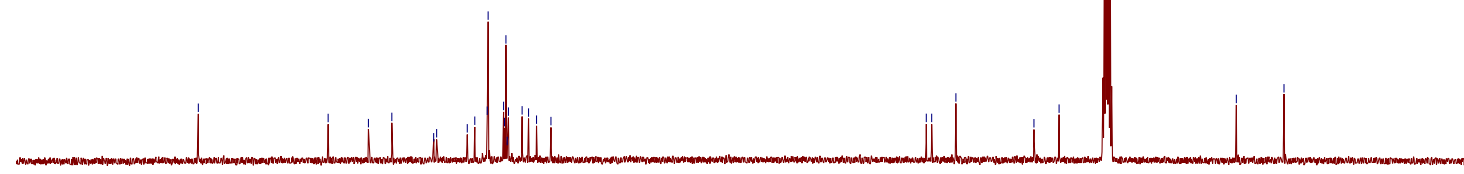

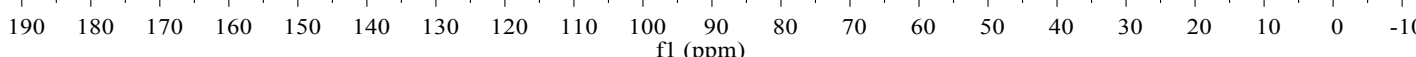




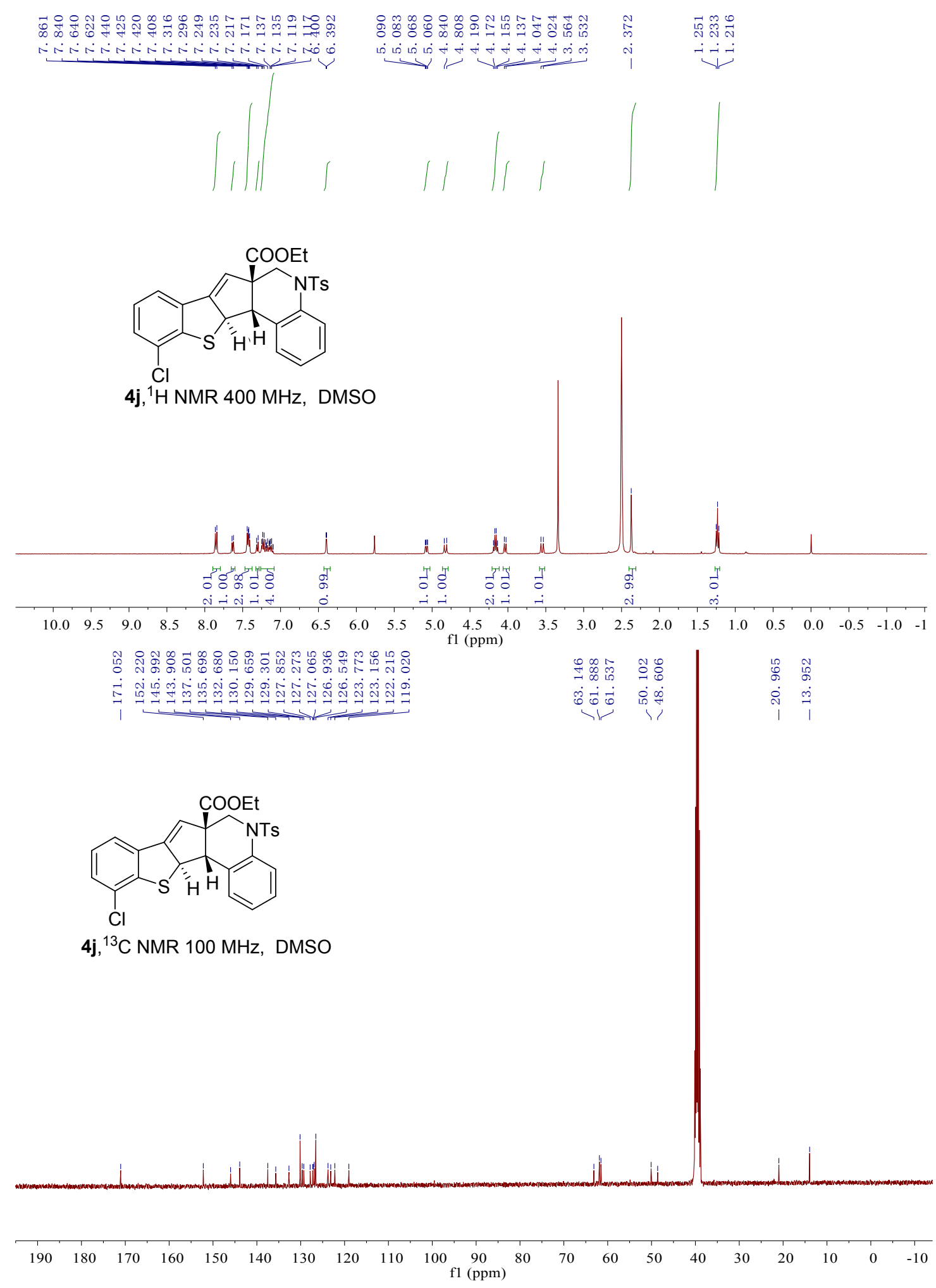



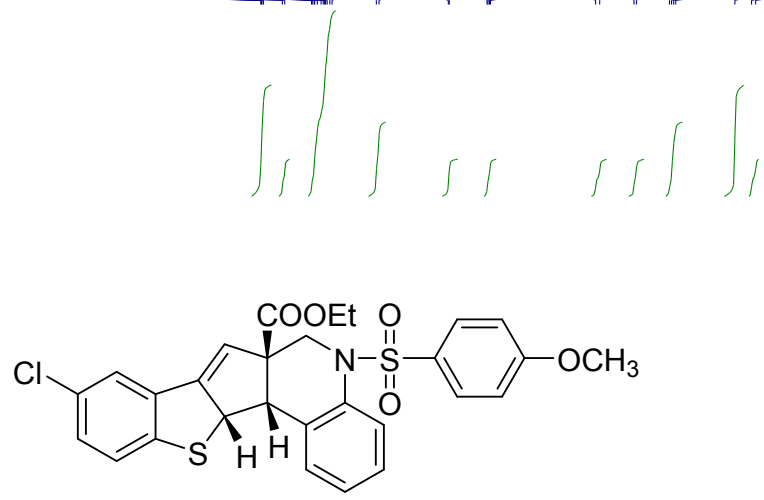

3k, ${ }^{1} \mathrm{H}$ NMR $400 \mathrm{MHz}$, DMSO

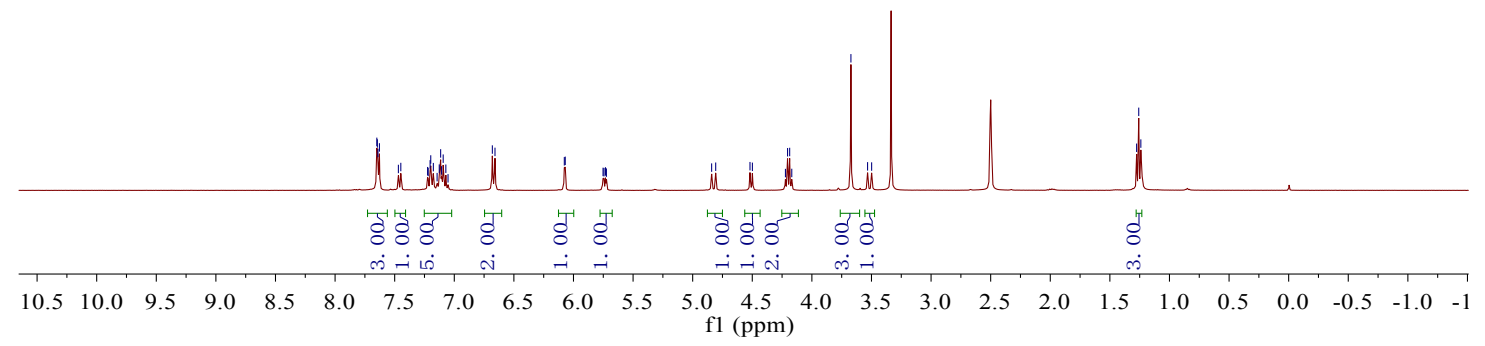

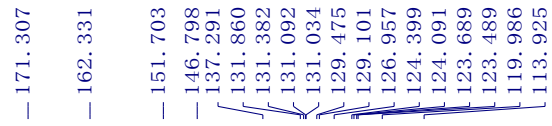
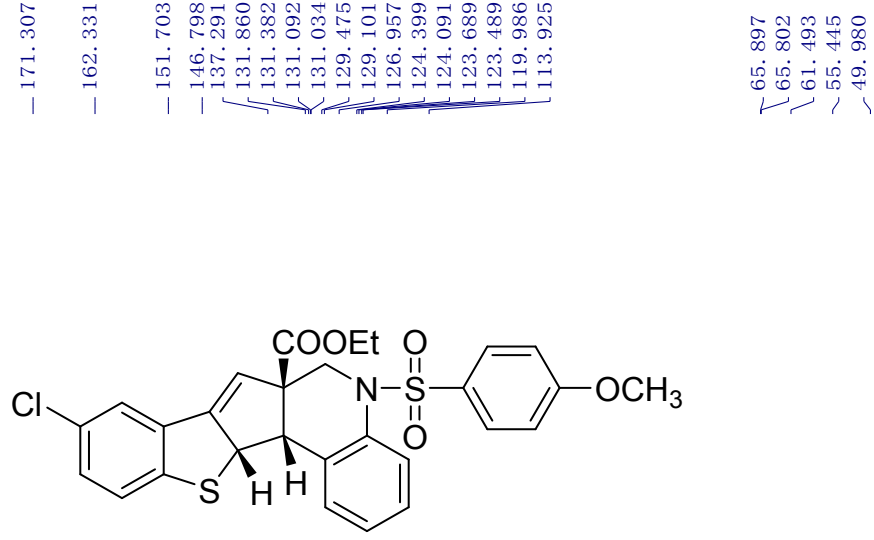

3k, ${ }^{1} \mathrm{H}$ NMR $400 \mathrm{MHz}$, DMSO

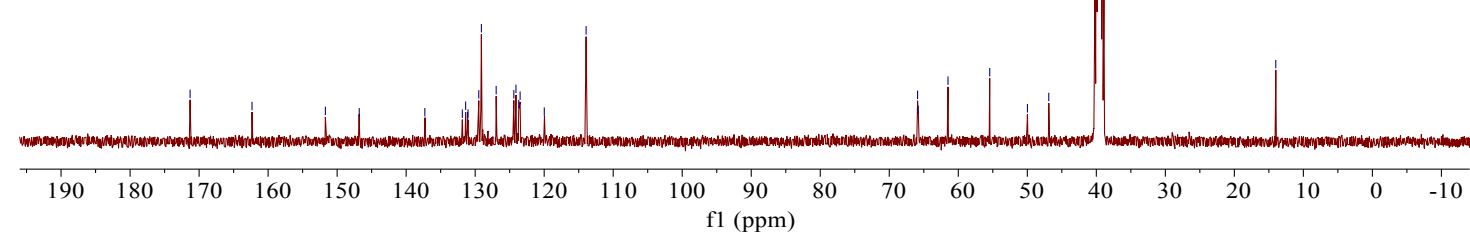




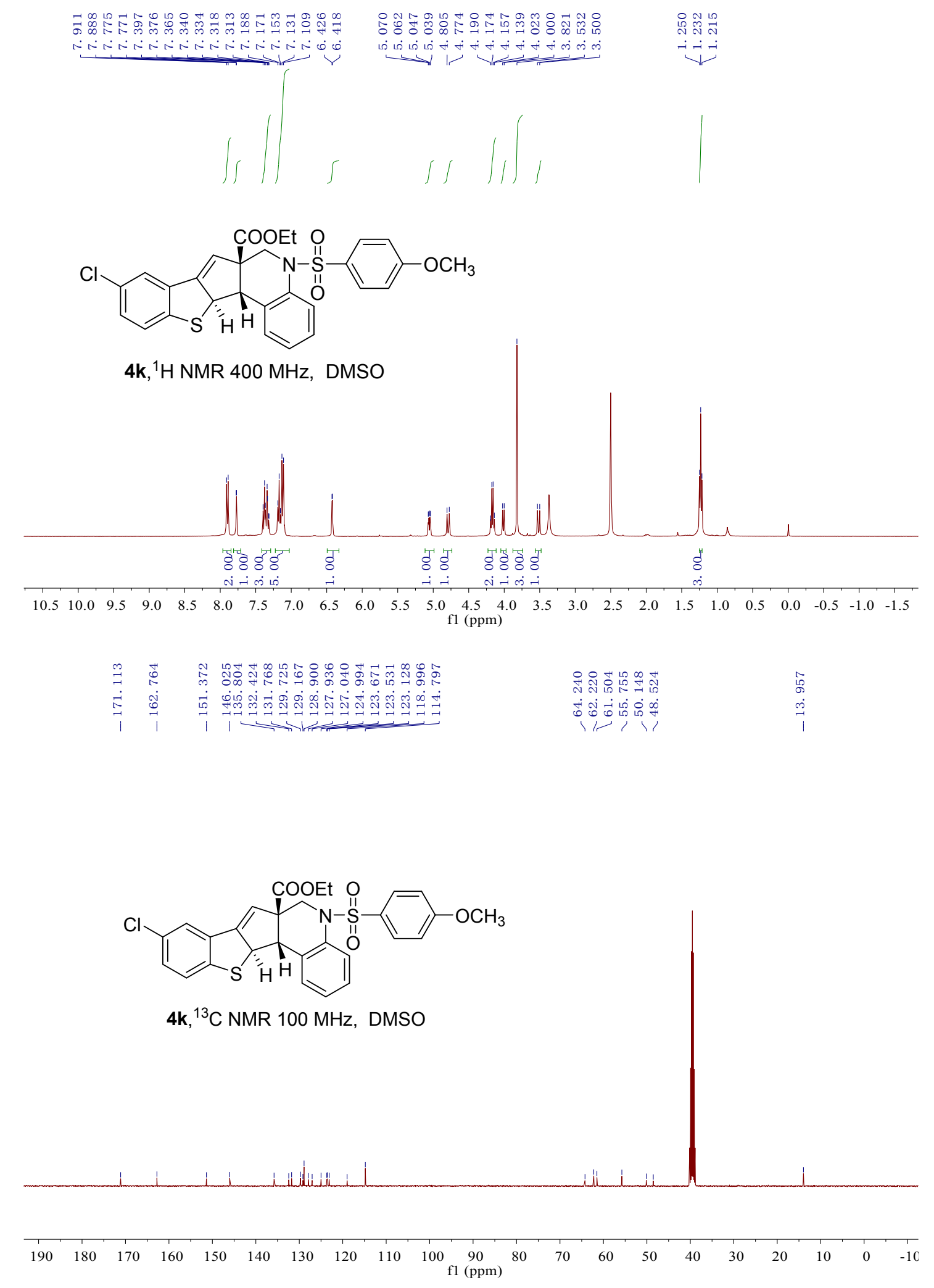



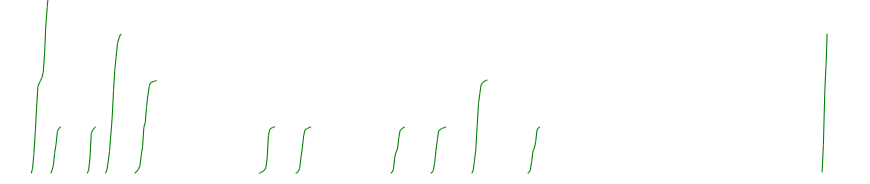<smiles>CCOCC12[C@@H]3Sc4ccc(Cl)cc4C3=CC1(C(=O)OCC)c1ccccc1N2S(=O)(=O)c1ccc([N+](=O)[O-])cc1</smiles>

31, ${ }^{1} \mathrm{H}$ NMR $400 \mathrm{MHz}$, DMSO
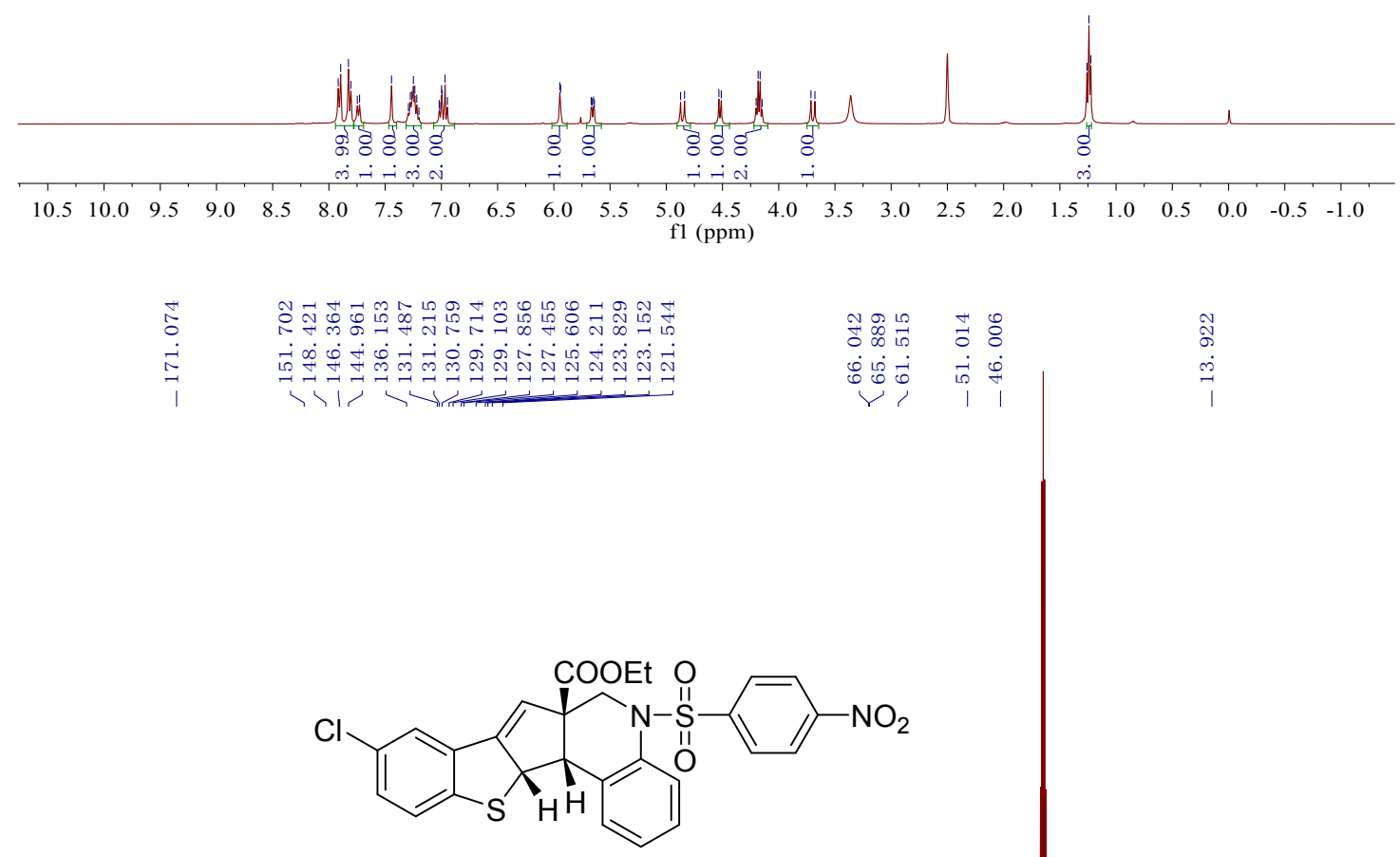

3I, ${ }^{13} \mathrm{C}$ NMR $100 \mathrm{MHz}$, DMSO

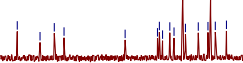

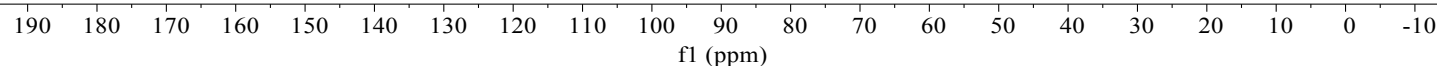


<smiles>CCOC(=O)C12C=C3c4cc(Cl)ccc4S[C@H]3C1c1ccccc1N(S(=O)(=O)c1ccc([N+](=O)[O-])cc1)O2</smiles>

4I, ${ }^{1} \mathrm{H}$ NMR $400 \mathrm{MHz}$, DMSO

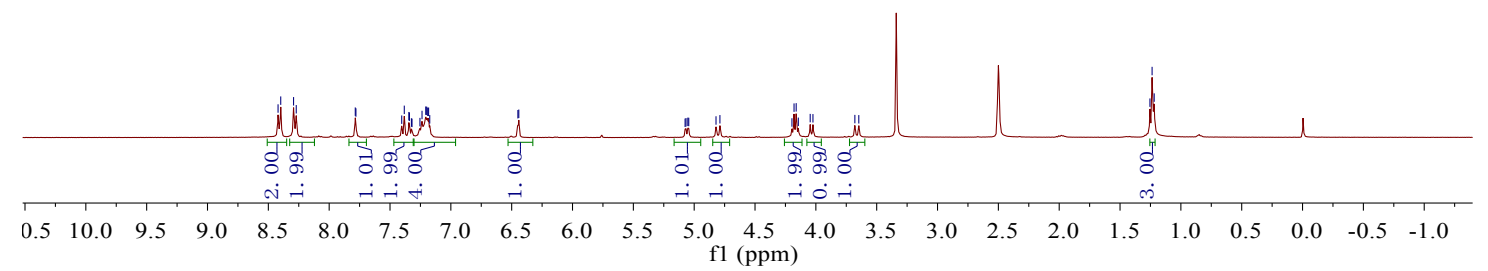<smiles>CCOC1(C)C=C2c3cc(Cl)ccc3S[C@H]2[C@@H]1c1ccccc1NS(=O)(=O)c1ccc([N+](=O)[O-])cc1</smiles>

4I, ${ }^{13} \mathrm{C}$ NMR $100 \mathrm{MHz}$, DMSO

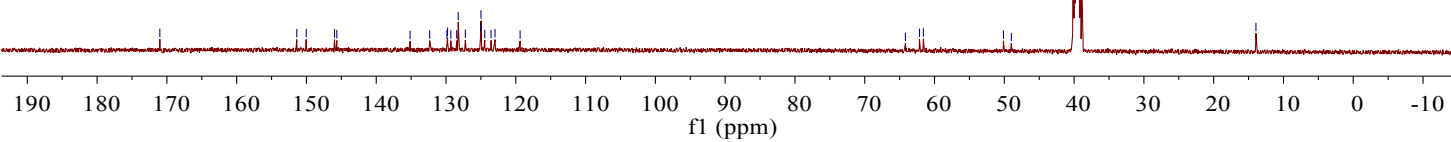



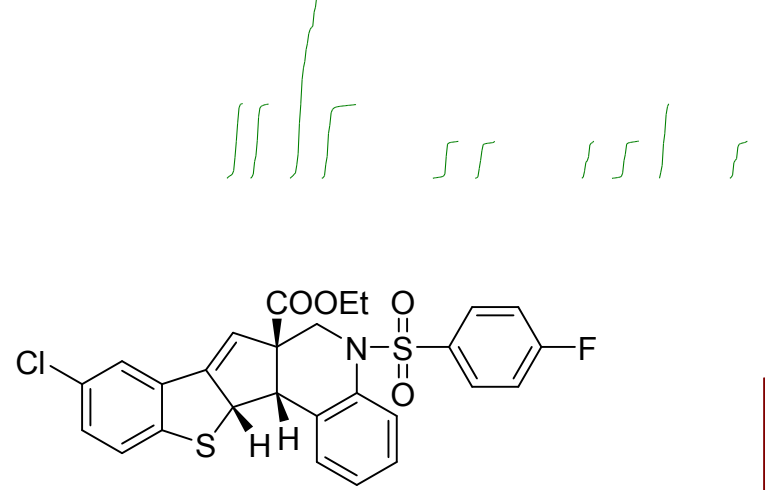

$3 m,{ }^{1} \mathrm{H}$ NMR $400 \mathrm{MHz}$, DMSO

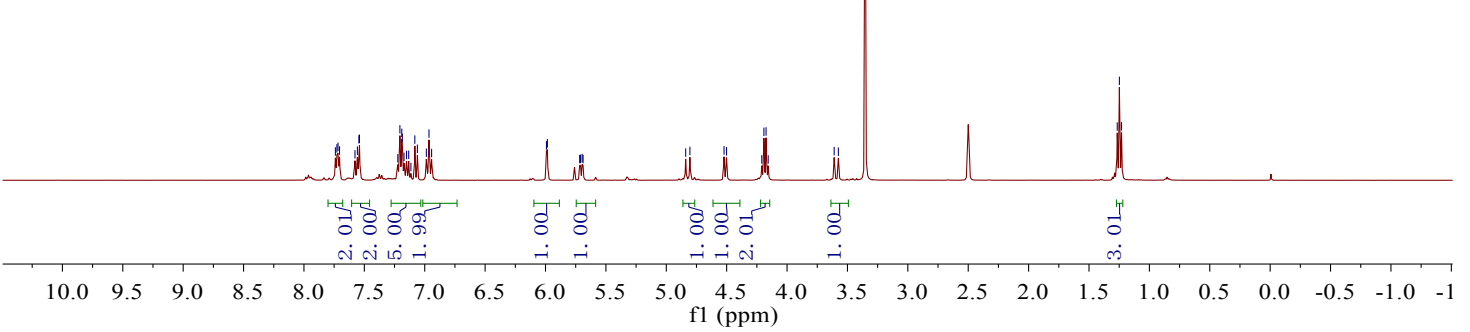

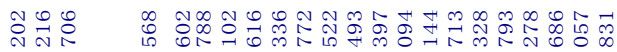

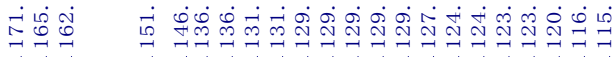

$1<1<1$<smiles>CCOC(=O)C1(C)CC2=C3c4cc(Cl)ccc4S[C@H]3[C@H]2c2ccccc2N1S(=O)(=O)c1ccc(F)cc1</smiles>

$3 m,{ }^{13} \mathrm{C}$ NMR $100 \mathrm{MHz}$, DMSO

$\begin{array}{lllllllllll}190 & 180 & 170 & 160 & 150 & 140 & 130 & 120 & 110 & 100 & 90 \\ \mathrm{f} 1(\mathrm{ppm}) & 80\end{array}$ 


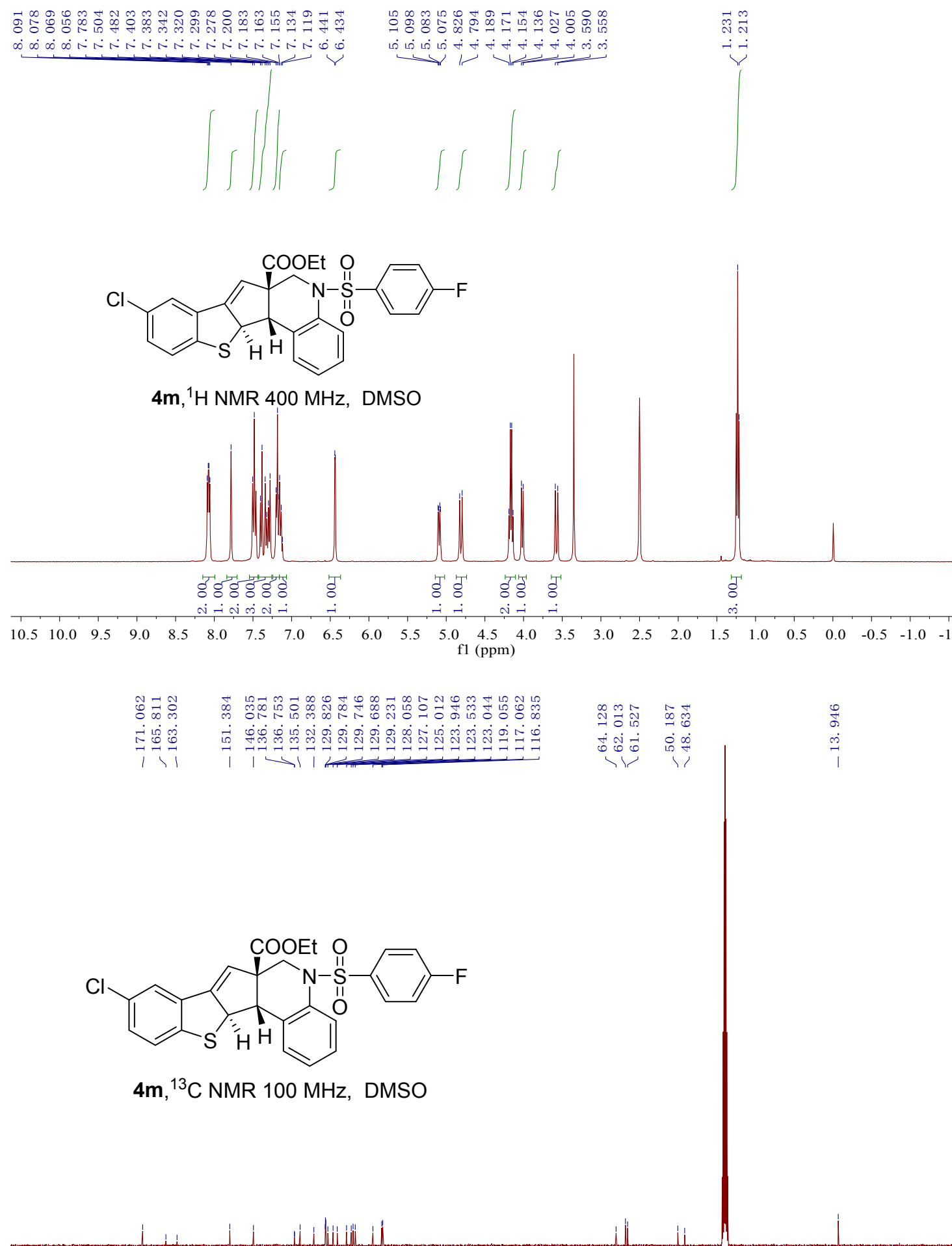

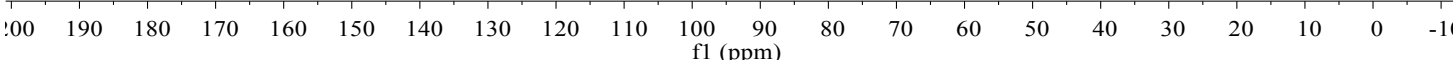



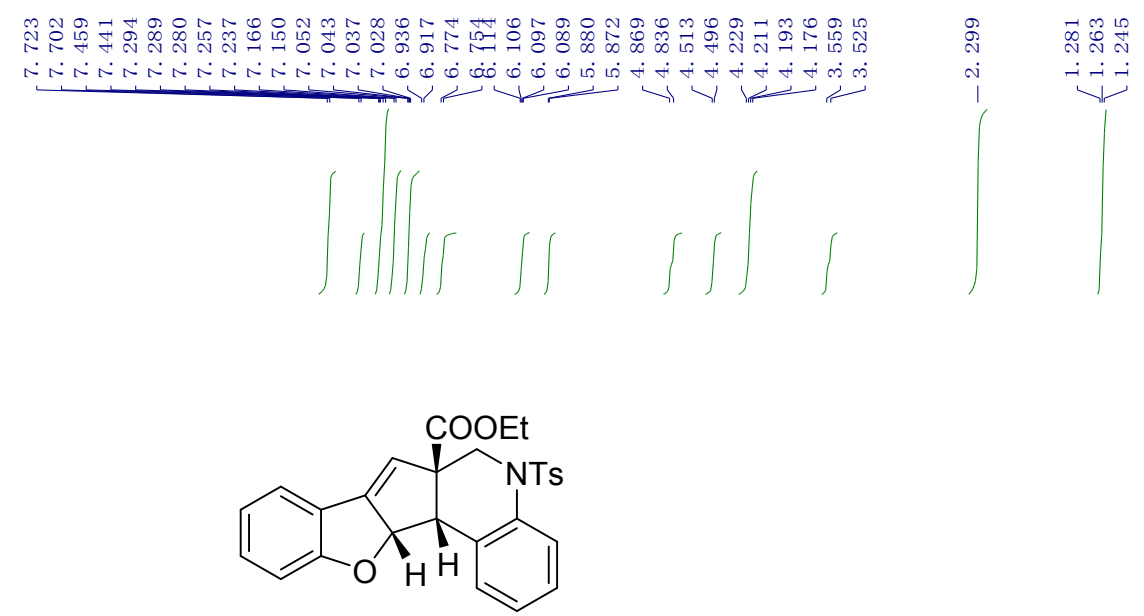

6a, ${ }^{1} \mathrm{H}$ NMR $400 \mathrm{MHz}$, DMSO
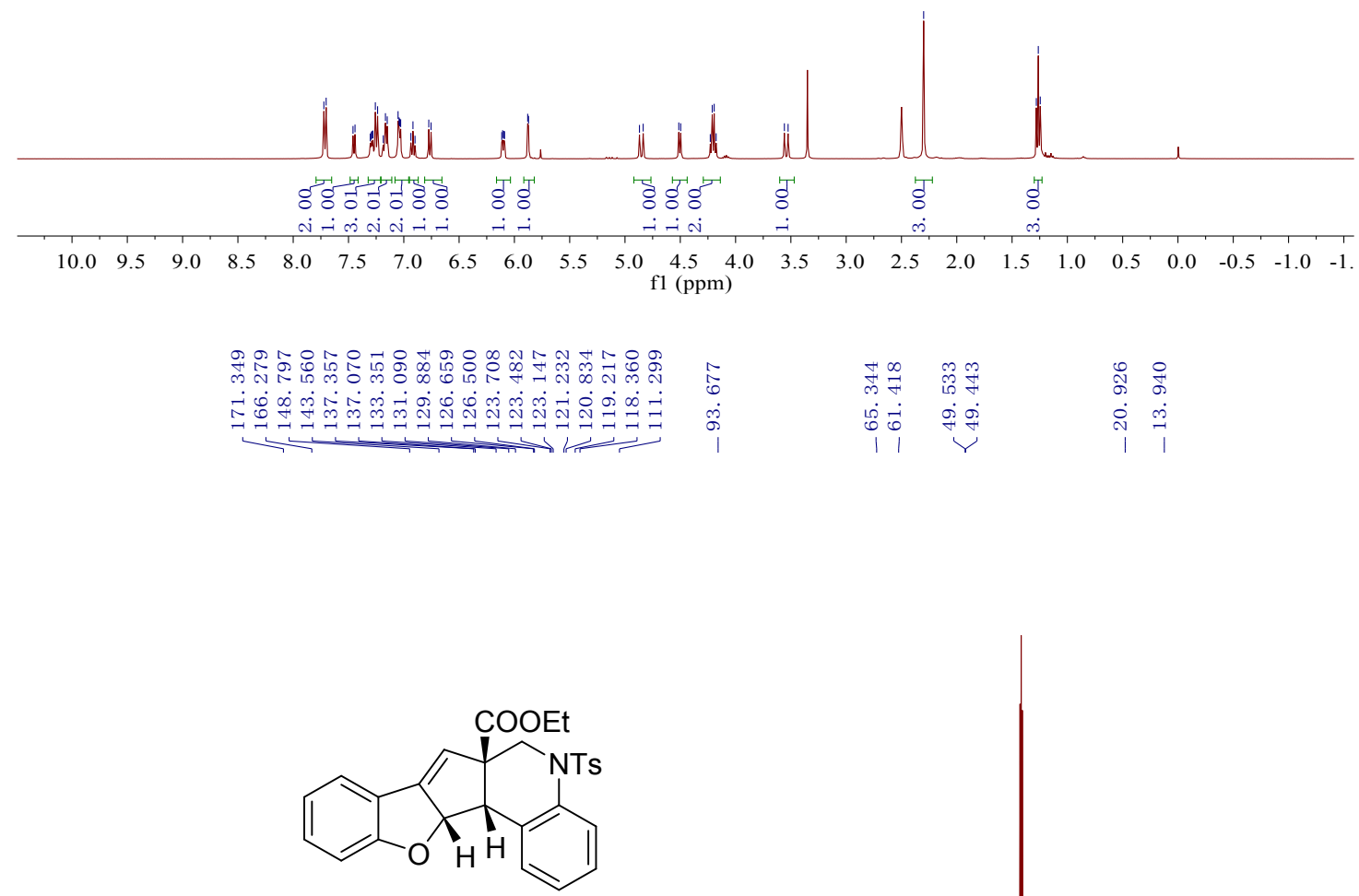

6a, ${ }^{13} \mathrm{C}$ NMR $100 \mathrm{MHz}$, DMSO

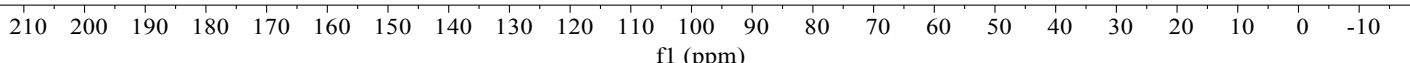




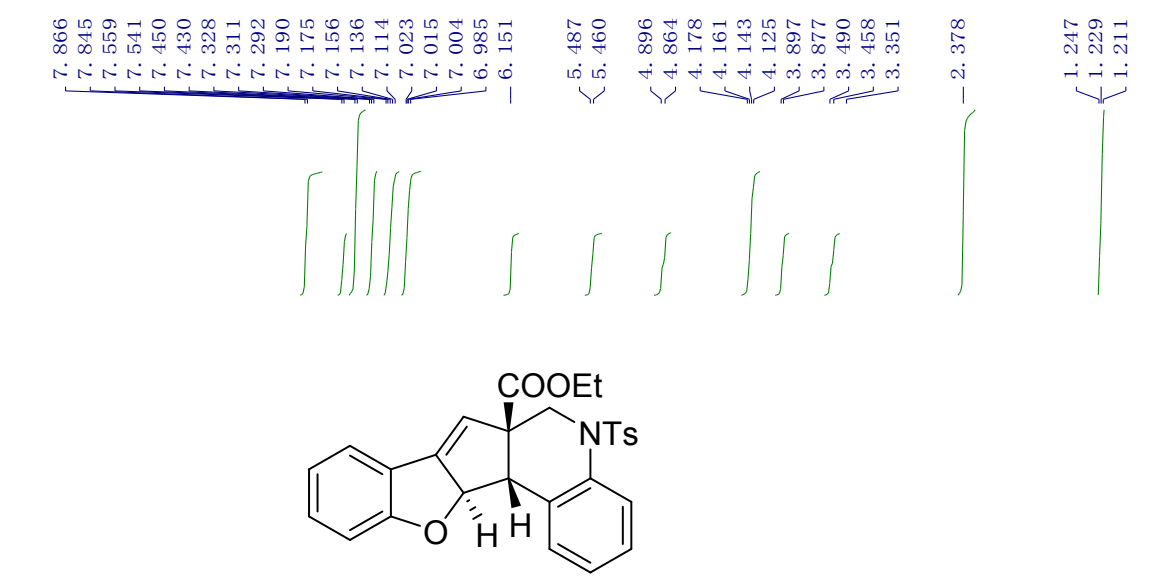

7a, ${ }^{1} \mathrm{H}$ NMR $400 \mathrm{MHz}$, DMSO

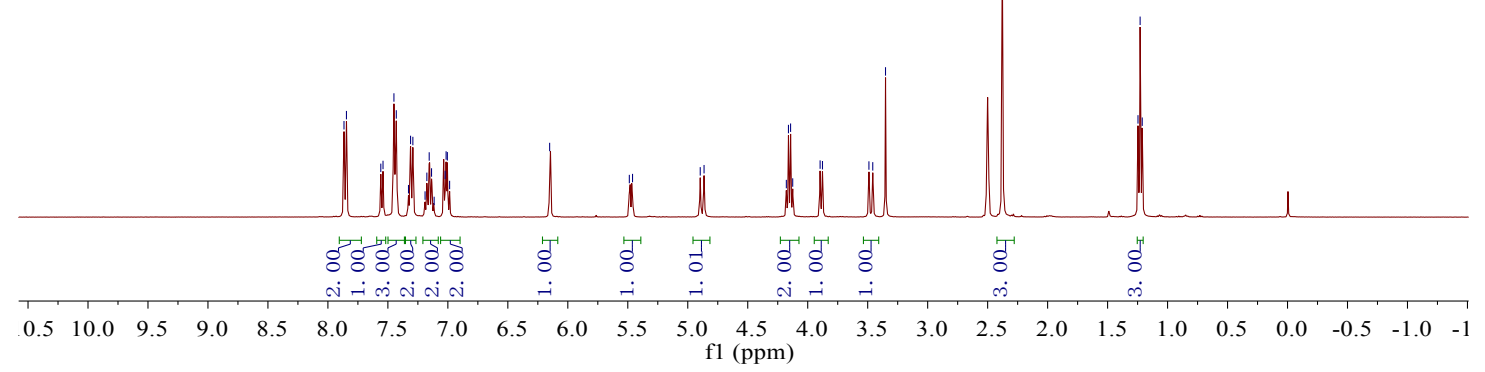

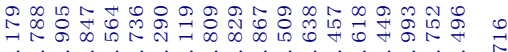

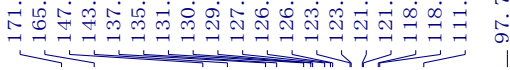<smiles>CCOC(=O)C12C=C3c4ccccc4OC3[C@H]1c1ccccc1[N+]2(C)[S-]</smiles>

7a, ${ }^{13} \mathrm{C}$ NMR $100 \mathrm{MHz}$, DMSO

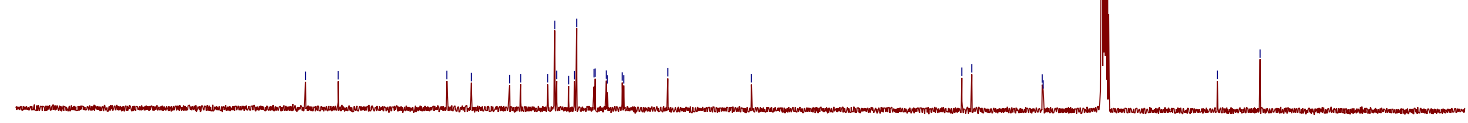

$\begin{array}{lllllllllllllllllllllll}210 & 200 & 190 & 180 & 170 & 160 & 150 & 140 & 130 & 120 & 110 & 100 & 90 & 80 & 70 & 60 & 50 & 40 & 30 & 20 & 10 & 0 & -10\end{array}$ 


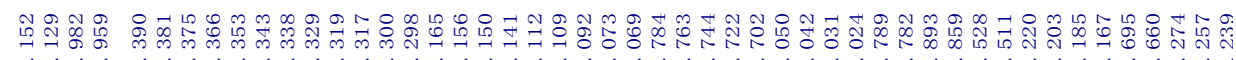
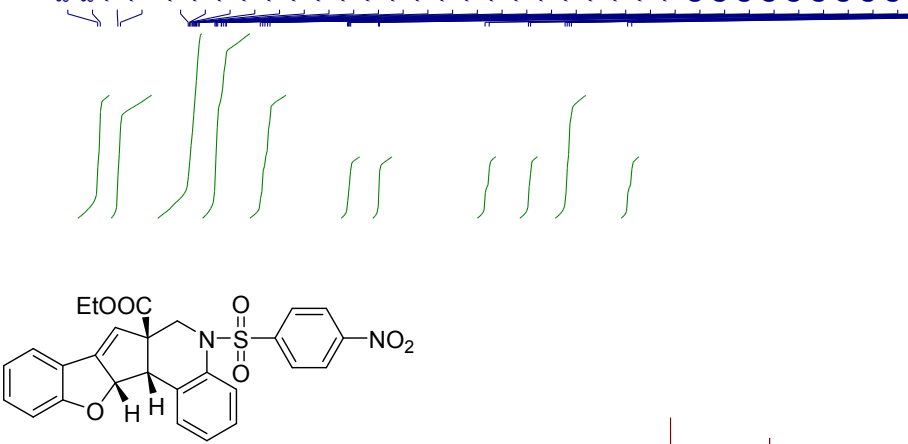

6b, ${ }^{1} \mathrm{H}$ NMR $400 \mathrm{MHz}$, DMSO

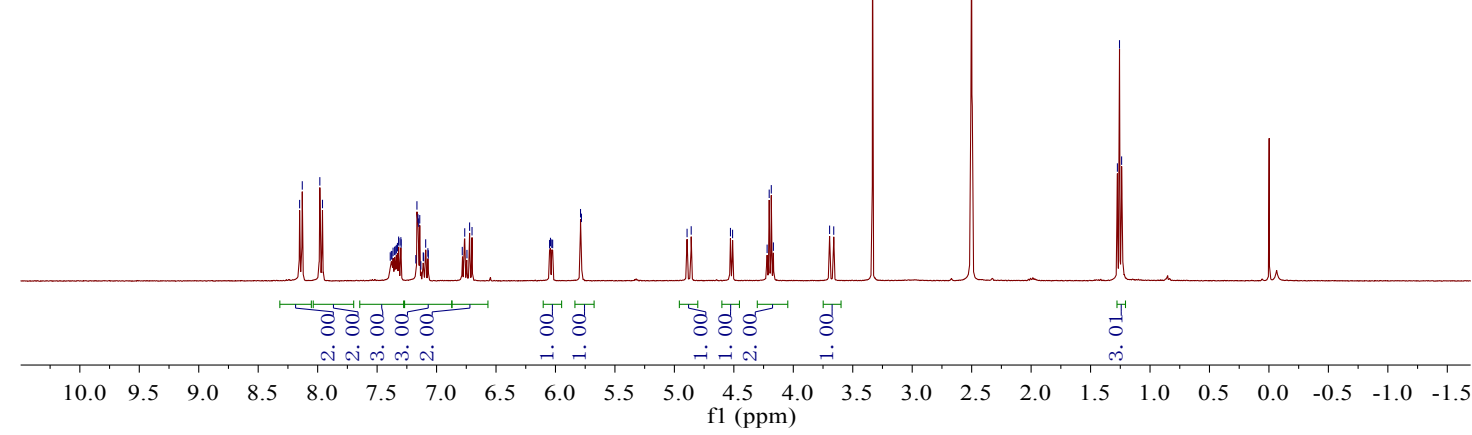

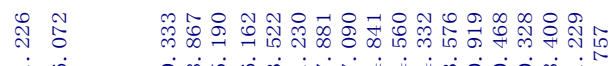

亡<smiles>COCCOC(=O)C1(CO)CN(S(=O)(=O)c2ccc([N+](=O)[O-])cc2)c2ccccc2C12COc1ccccc12</smiles>

6b, ${ }^{13} \mathrm{C}$ NMR $100 \mathrm{MHz}$, DMSO
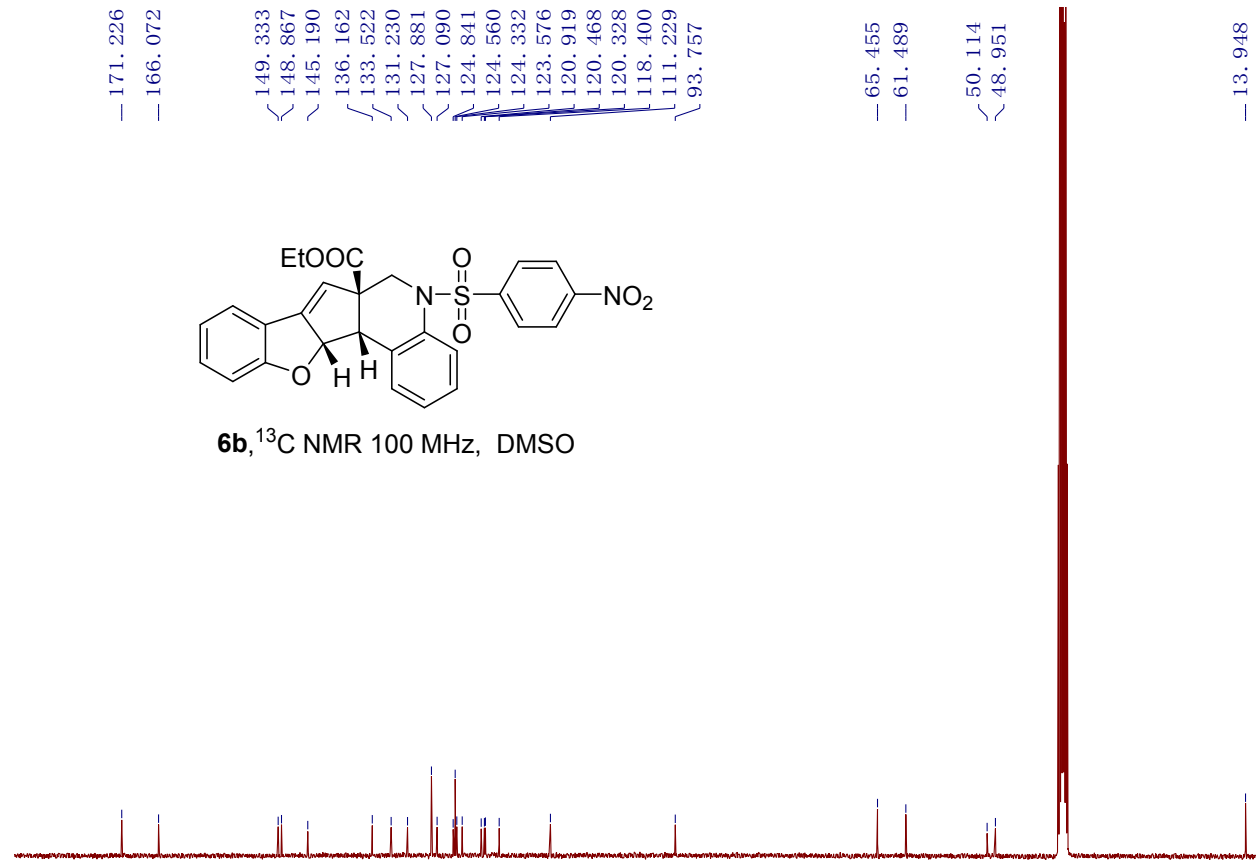

$\begin{array}{rrrrrrrrrrrrrrrrrrr}180 & 170 & 160 & 150 & 140 & 130 & 120 & 110 & 100 & \begin{array}{r}90 \\ \mathrm{f} 1(\mathrm{ppm})\end{array} & 70 & 60 & 50 & 40 & 30 & 20 & 10 & 0 & -10\end{array}$ 


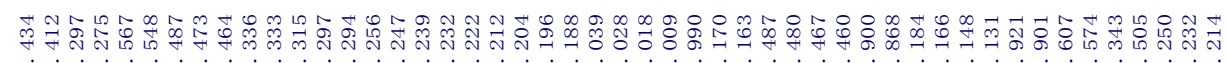

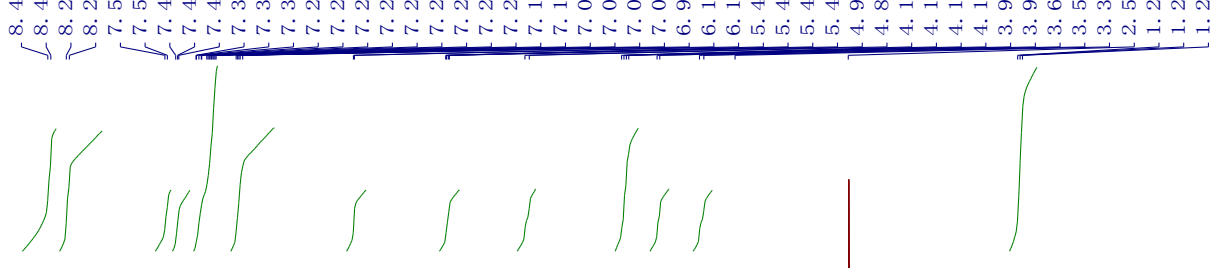<smiles>CCOC(=O)C12CN(S(=O)(=O)c3ccc([N+](=O)[O-])cc3)c3ccccc3C1C1Oc3ccccc3OC12</smiles>

7b, ${ }^{1}$ H NMR 400 MHz, DMSO

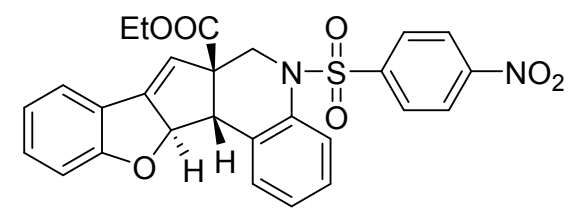

7b, ${ }^{13} \mathrm{C}$ NMR $100 \mathrm{MHz}$, DMSO

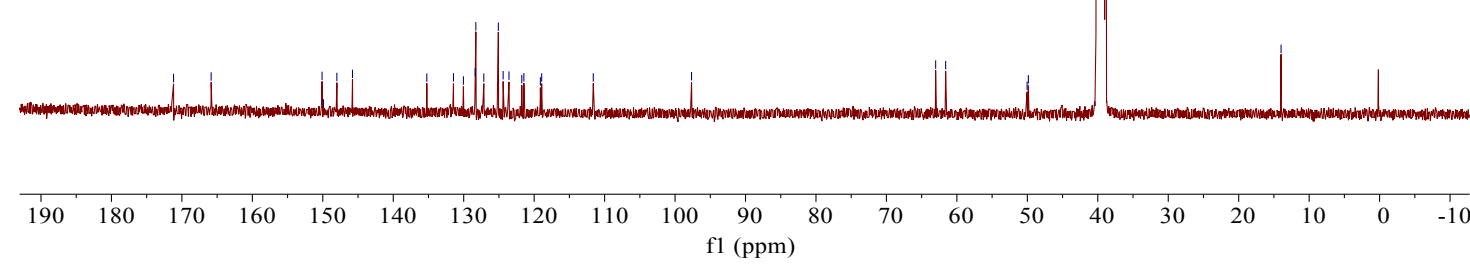




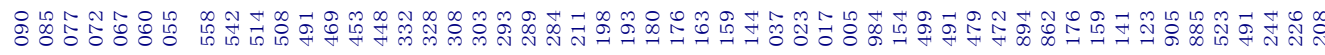

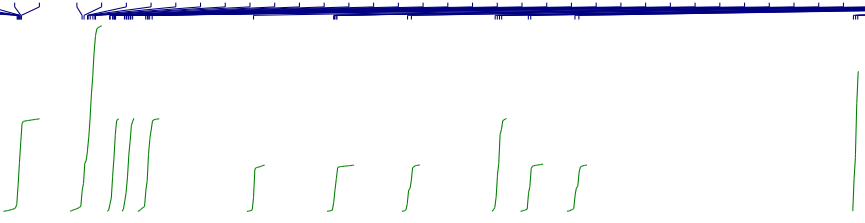<smiles>CCOCC12CC3(OCc4ccccc43)C1(COCC)CN(S(=O)(=O)c1ccc(F)cc1)c1ccccc12</smiles>

7c, ${ }^{1} \mathrm{H}$ NMR $400 \mathrm{MHz}$, DMSO

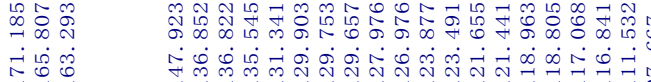

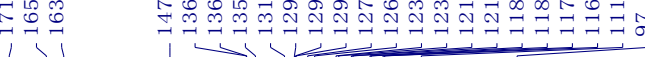<smiles>CCOCC12C=C3c4ccccc4OC3C1c1ccccc1N(S(=O)(=O)c1ccc(F)cc1)C2</smiles>

7c, ${ }^{13} \mathrm{C}$ NMR $100 \mathrm{MHz}$, DMSO

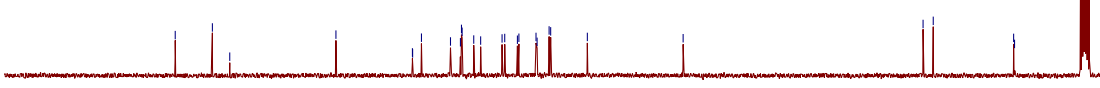

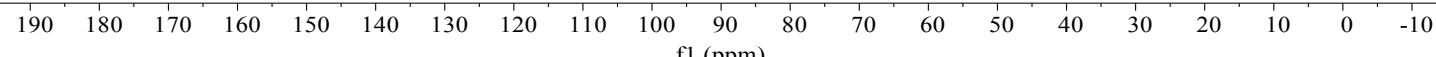




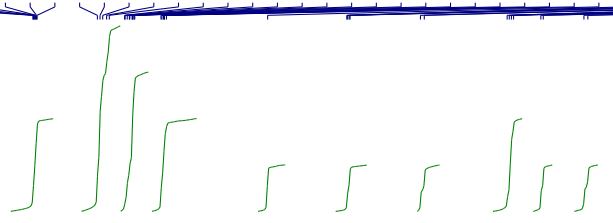<smiles>CCOC[C@]1(C)CN(S(=O)(=O)c2ccc(F)cc2)c2ccc(Cl)cc2[C@H]1[C@H]1Oc2ccccc2C1=O</smiles>

7d, ${ }^{1} \mathrm{H}$ NMR $400 \mathrm{MHz}$, DMSO

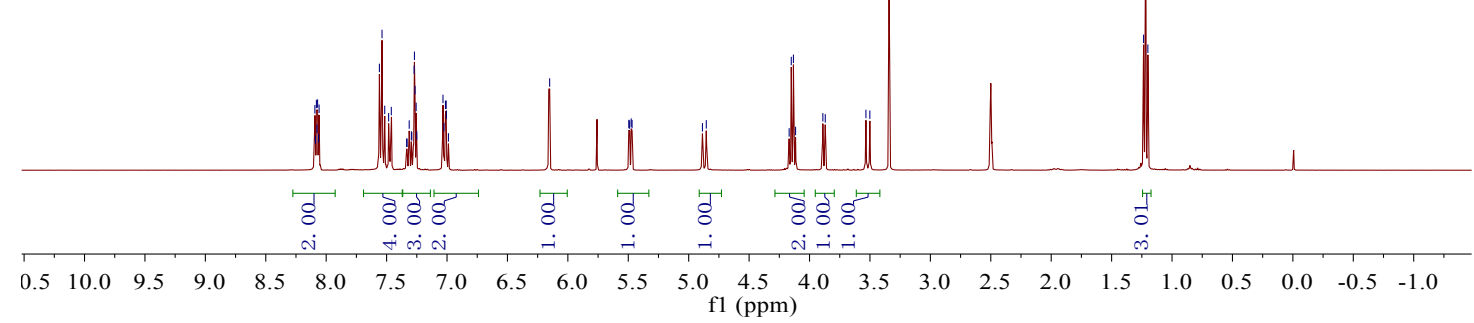

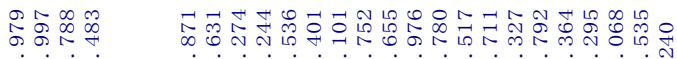

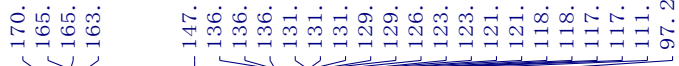

ㄴ.<smiles>CCOC[C@]12C=C3c4ccccc4O[C@H]3[C@@H]1c1ccc(Cl)cc1N(S(=O)(=O)c1ccc(F)cc1)C2</smiles>

7d, ${ }^{13} \mathrm{C}$ NMR $100 \mathrm{MHz}$, DMSO

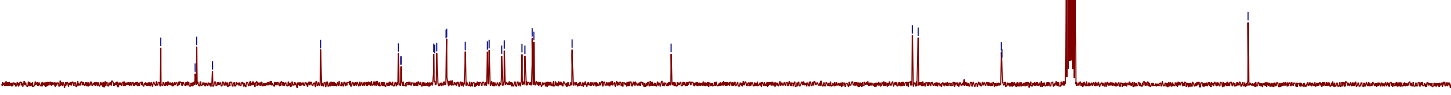

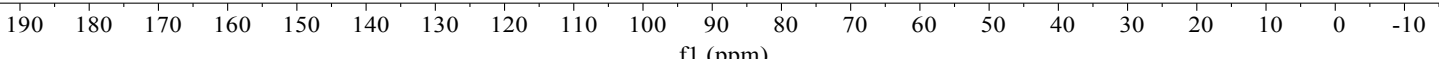



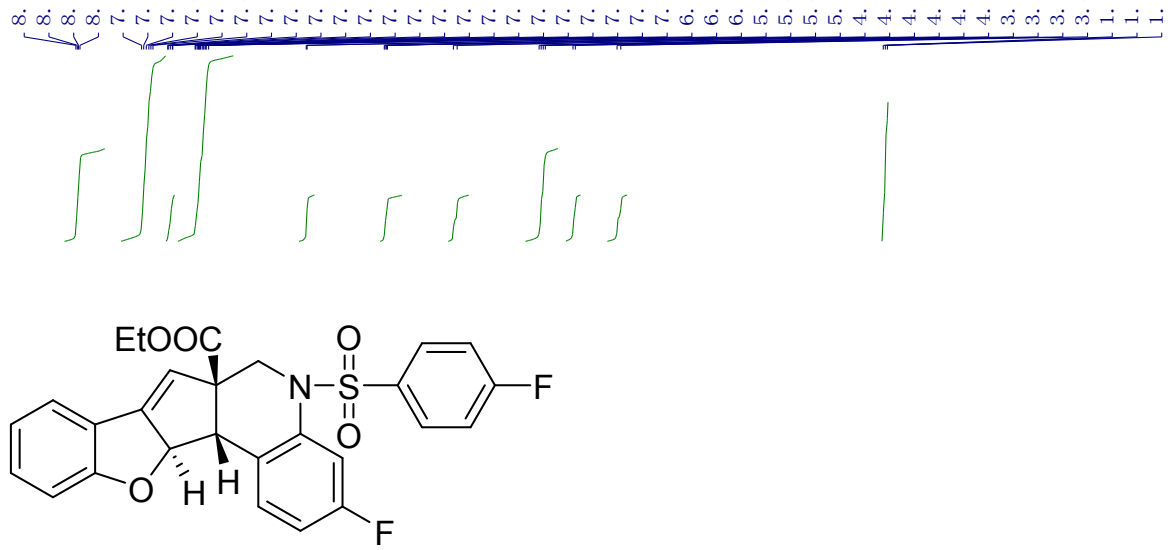

7e, ${ }^{1} \mathrm{H}$ NMR $400 \mathrm{MHz}$, DMSO
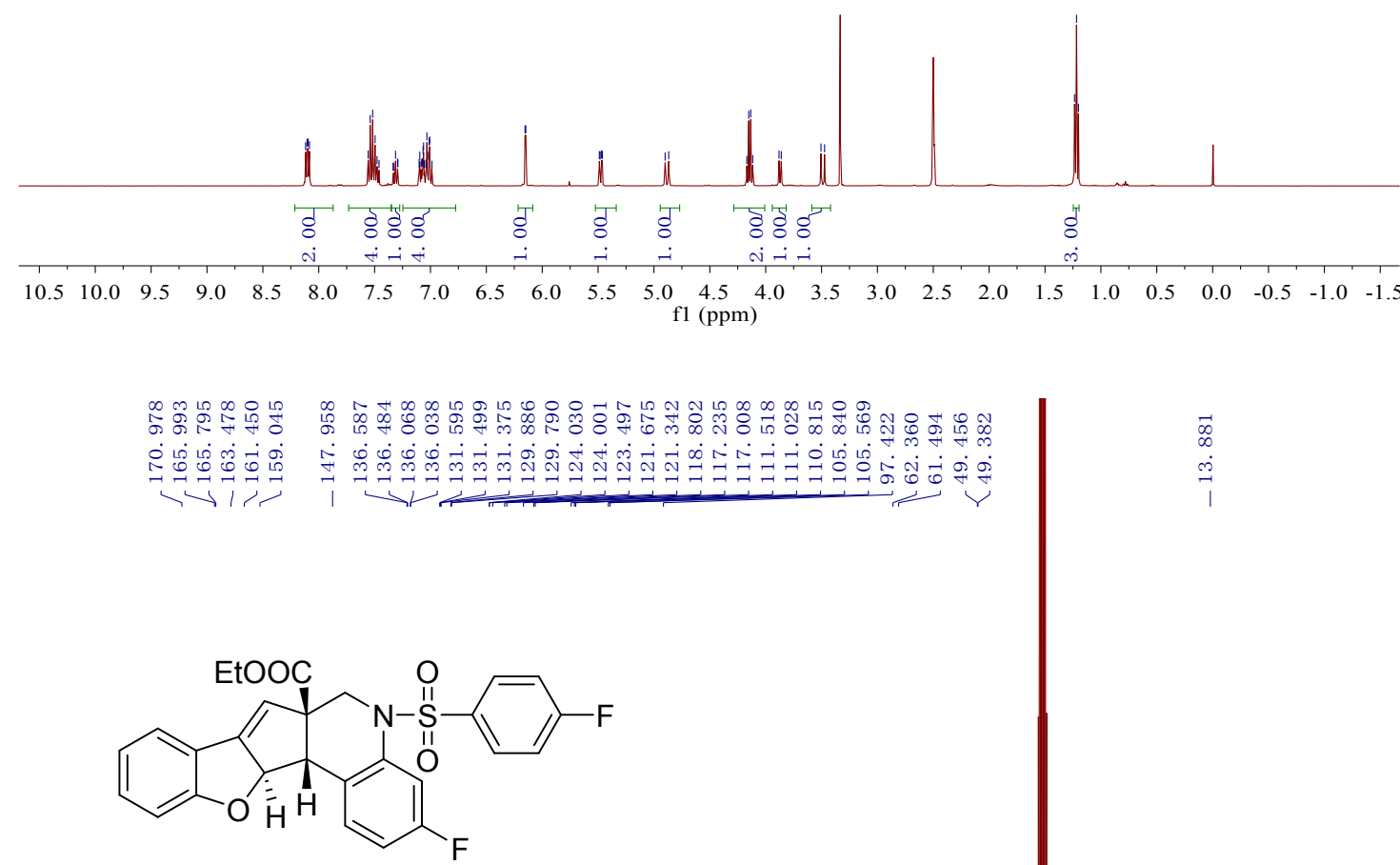

7e, ${ }^{13} \mathrm{C}$ NMR $100 \mathrm{MHz}$, DMSO
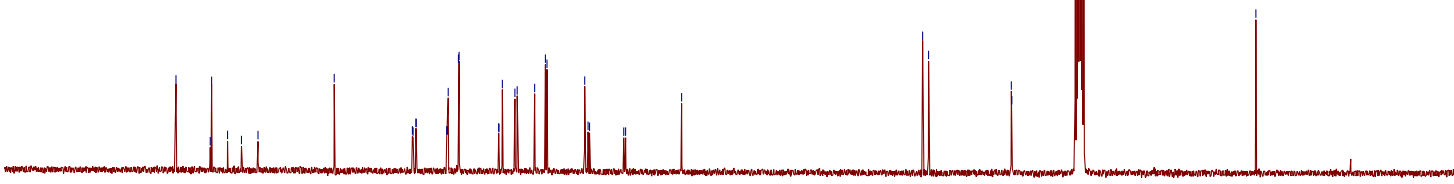

$\begin{array}{rllllllllllllllllllll}190 & 180 & 170 & 160 & 150 & 140 & 130 & 120 & 110 & 100 & \begin{array}{c}90 \\ \mathrm{fl}(\mathrm{ppm})\end{array} & 80 & 70 & 60 & 50 & 40 & 30 & 20 & 10 & 0 & -10\end{array}$ 

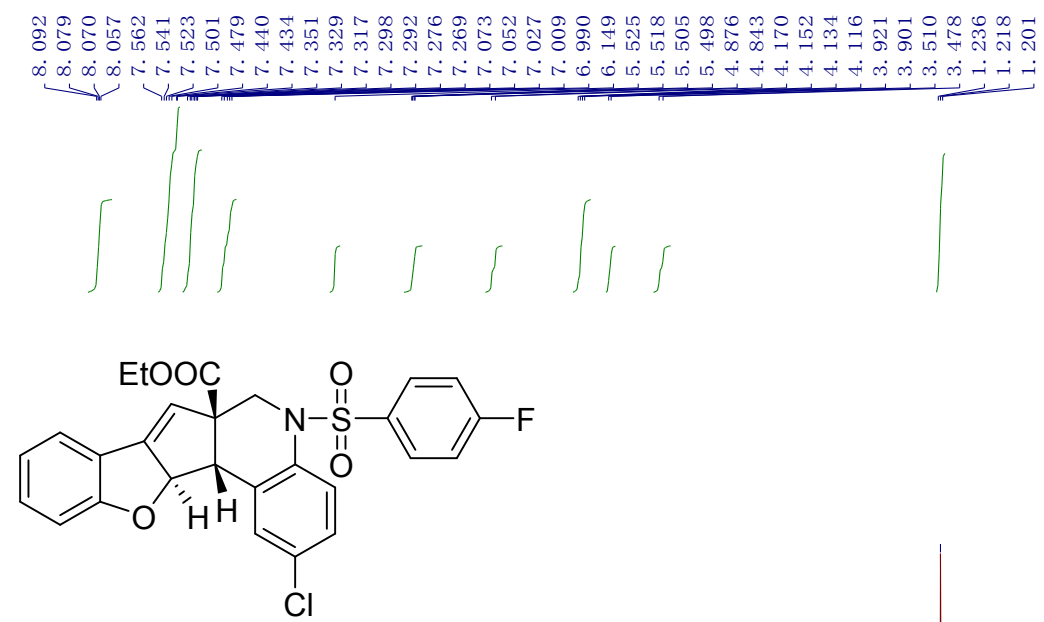

7f, ${ }^{1} \mathrm{H}$ NMR $400 \mathrm{MHz}$, DMSO

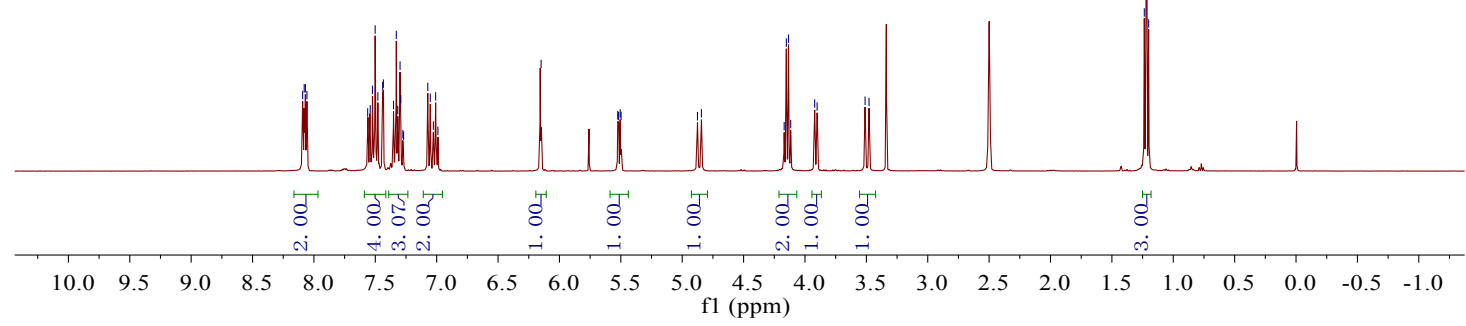

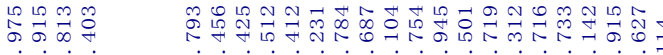

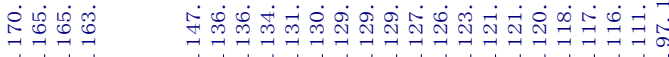<smiles>CCOC[C@]12C=C3c4ccccc4O[C@H]3[C@H]1c1cc(Cl)ccc1N(S(=O)(=O)c1ccc(F)cc1)C2</smiles>

7f, ${ }^{13} \mathrm{C}$ NMR $100 \mathrm{MHz}$, DMSO

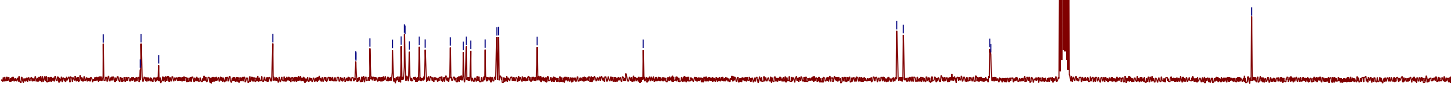

$\begin{array}{rrrrrrrrlll}180 & 170 & 160 & 150 & 140 & 130 & 120 & 110 & 100 & 90 & 80\end{array}$ 

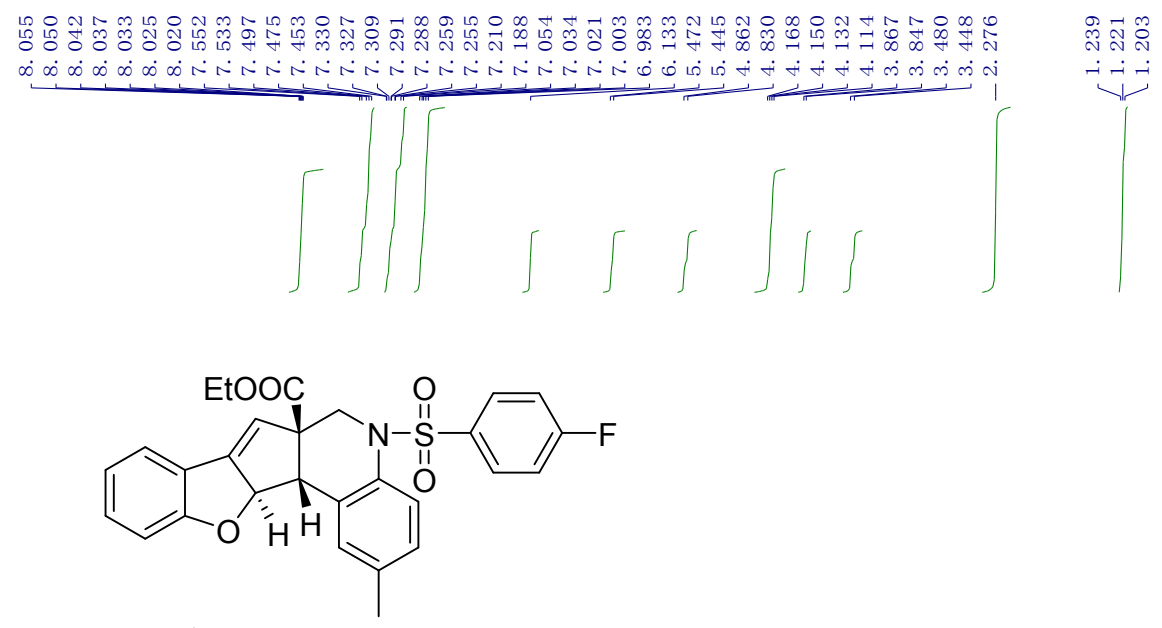

7g, ${ }^{1} \mathrm{H}$ NMR $400 \mathrm{MHz}$, DMSO

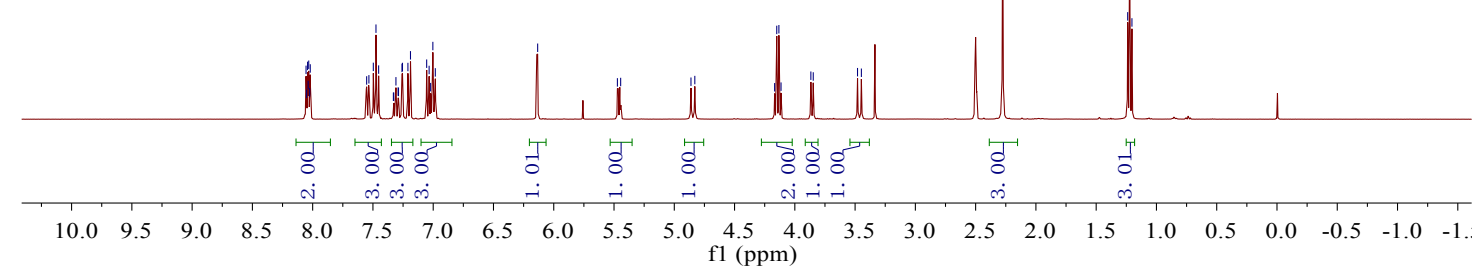

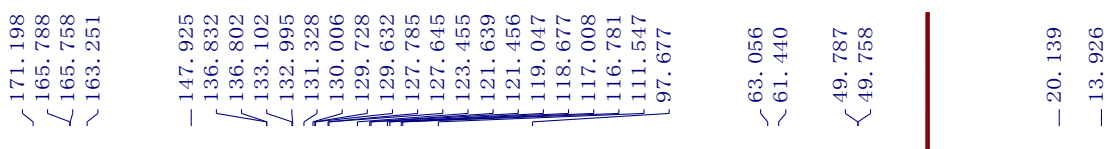<smiles>CCOC[C@@]12C=C3c4ccccc4O[C@H]3[C@H]1c1cc(C)ccc1N(S(=O)(=O)c1ccc(F)cc1)C2</smiles>

7g, ${ }^{13} \mathrm{C}$ NMR $100 \mathrm{MHz}$, DMSO

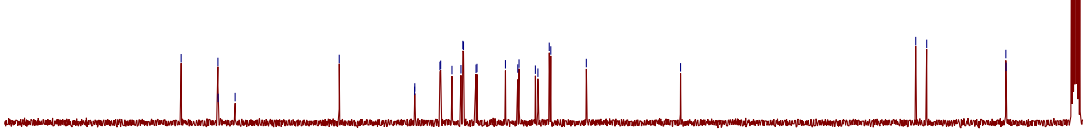

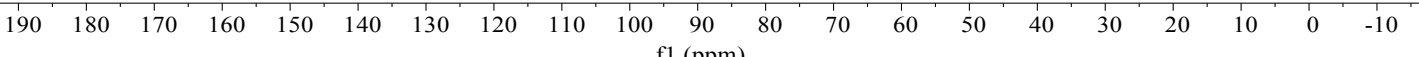
f1 (ppm) 


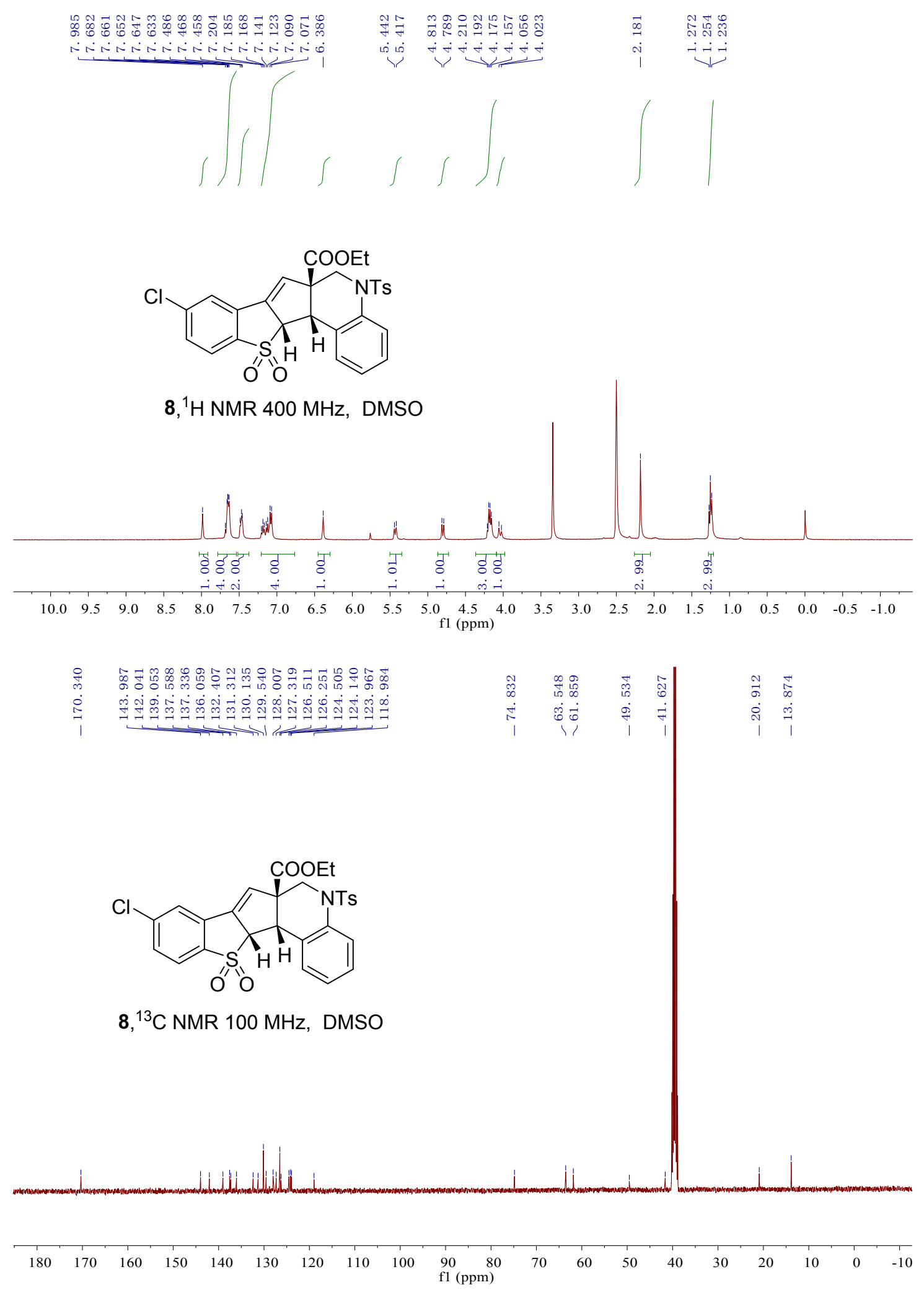



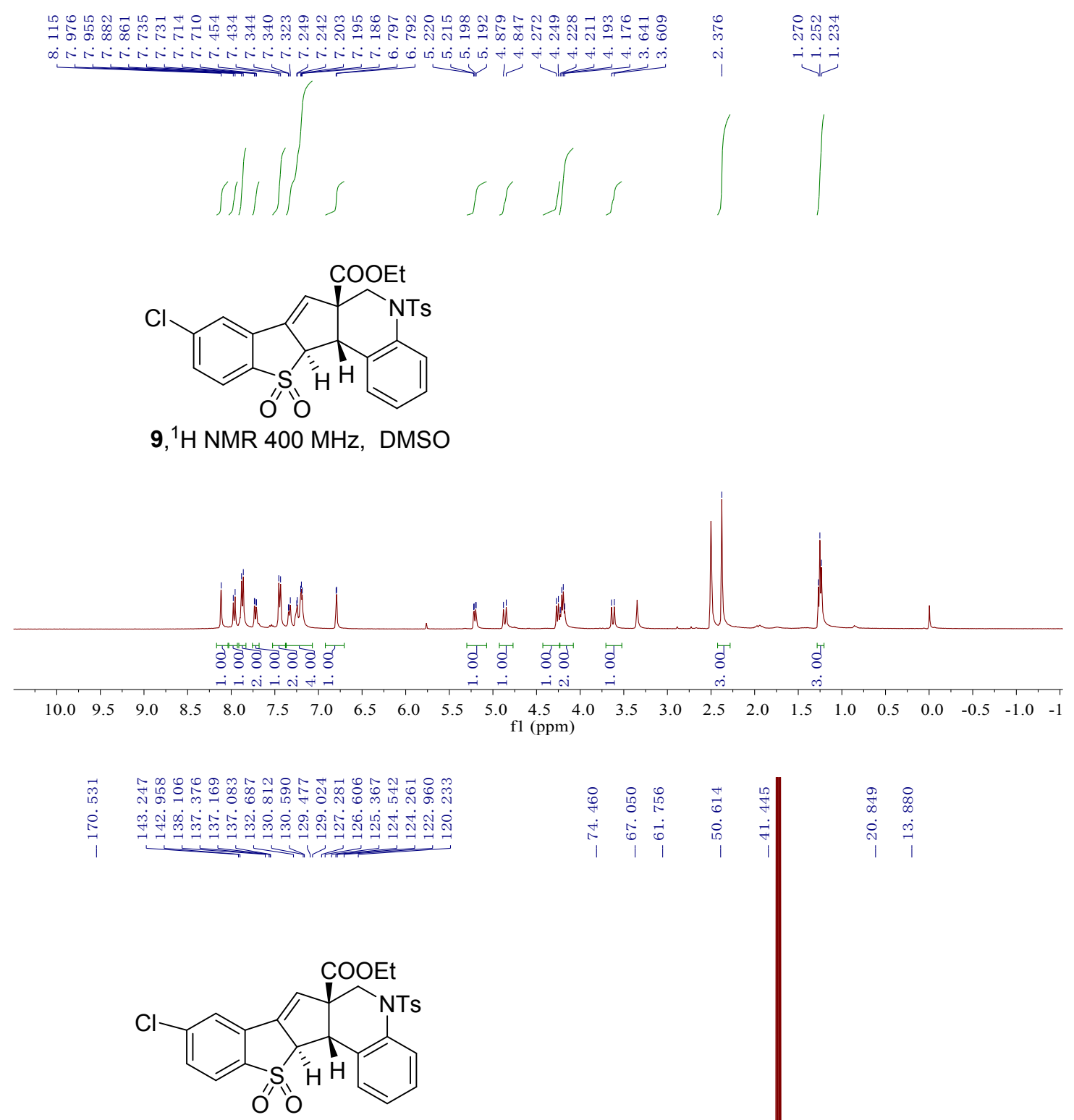

9, ${ }^{13} \mathrm{C}$ NMR $100 \mathrm{MHz}$, DMSO

$\begin{array}{lllllllllllllllllll}180 & 170 & 160 & 150 & 140 & 130 & 120 & 110 & 100 & \begin{array}{l}90 \\ \mathrm{f} 1(\mathrm{ppm})\end{array} & 70 & 60 & 50 & 40 & 30 & 20 & 10 & 0 & -10\end{array}$ 


\section{Computational details and archive entries}

Table S1. Cartesian coordinates of some stationary points, optimized at the m06$\mathrm{PCM} / 6-31 \mathrm{G}^{*}$ level of theory in $\mathrm{CH}_{3} \mathrm{CN}$.

\begin{tabular}{|c|c|c|c|c|}
\hline Species Name & & sian Coordinat & & \\
\hline & 6 & 4.091398000 & -0.847481000 & 0.275202000 \\
\hline & 6 & 3.634232000 & 0.410222000 & -0.118455000 \\
\hline & 6 & 4.502790000 & 1.494614000 & -0.208639000 \\
\hline & 6 & 5.834173000 & 1.283250000 & 0.104638000 \\
\hline & 6 & 6.302537000 & 0.027851000 & 0.502055000 \\
\hline & 6 & 5.431093000 & -1.050514000 & 0.589376000 \\
\hline & 6 & 1.606118000 & -0.908549000 & -0.202156000 \\
\hline & 6 & 2.197330000 & 0.445836000 & -0.414948000 \\
\hline & 1 & 4.129829000 & 2.468542000 & -0.520308000 \\
\hline & 1 & 7.355503000 & -0.099999000 & 0.741723000 \\
\hline & 1 & 5.798827000 & -2.026448000 & 0.898120000 \\
\hline & 8 & 1.566057000 & 1.426667000 & -0.777171000 \\
\hline & 16 & 2.829295000 & -2.088050000 & 0.311306000 \\
\hline & 6 & 0.279759000 & -1.100730000 & -0.345465000 \\
\hline & 1 & -0.273387000 & -0.191341000 & -0.596107000 \\
\hline & 17 & 6.966842000 & 2.614410000 & 0.002009000 \\
\hline & 6 & -0.505147000 & -2.312637000 & -0.205346000 \\
\hline & 6 & -1.876745000 & -2.216439000 & 0.118010000 \\
\hline & 6 & 0.033068000 & -3.590536000 & -0.422113000 \\
\hline & 6 & -2.662587000 & -3.361204000 & 0.214207000 \\
\hline & 6 & -0.756682000 & -4.727246000 & -0.347368000 \\
\hline & 1 & 1.077851000 & -3.688243000 & -0.711988000 \\
\hline $1 \mathrm{a}$ & 6 & -2.107698000 & -4.611074000 & -0.032044000 \\
\hline & 1 & -3.708269000 & -3.274880000 & 0.501905000 \\
\hline & 1 & -0.319075000 & -5.704113000 & -0.539403000 \\
\hline & 1 & -2.732355000 & -5.498730000 & 0.038598000 \\
\hline & 7 & -2.407798000 & -0.919335000 & 0.358887000 \\
\hline & 1 & -2.065207000 & -0.438208000 & 1.190462000 \\
\hline & 16 & -4.015838000 & -0.552239000 & 0.032189000 \\
\hline & 8 & -4.273950000 & -1.049778000 & -1.314309000 \\
\hline & 8 & -4.900783000 & -0.981533000 & 1.113578000 \\
\hline & 6 & -3.920413000 & 1.209837000 & 0.045547000 \\
\hline & 6 & -4.570456000 & 1.926129000 & 1.043016000 \\
\hline & 6 & -3.202657000 & 1.851037000 & -0.964956000 \\
\hline & 6 & -4.492088000 & 3.314002000 & 1.027426000 \\
\hline & 1 & -5.127124000 & 1.402741000 & 1.816607000 \\
\hline & 6 & -3.137496000 & 3.233797000 & -0.962346000 \\
\hline & 1 & -2.704875000 & 1.270054000 & -1.739606000 \\
\hline & 6 & -3.777415000 & 3.984201000 & 0.033356000 \\
\hline & 1 & -4.997125000 & 3.888444000 & 1.802759000 \\
\hline & 1 & -2.583244000 & 3.749444000 & -1.745830000 \\
\hline & 6 & -3.678356000 & 5.477872000 & 0.030947000 \\
\hline & 1 & -2.668927000 & 5.802371000 & 0.317699000 \\
\hline & 1 & -3.872454000 & 5.888876000 & -0.967487000 \\
\hline & 1 & -4.386145000 & 5.930219000 & 0.734117000 \\
\hline & 6 & -1.737915000 & -1.953765000 & -0.197906000 \\
\hline & 6 & -1.755759000 & -0.631768000 & -0.027361000 \\
\hline & 1 & -2.661883000 & -2.518342000 & -0.281234000 \\
\hline 2 & 1 & -0.801844000 & -2.501715000 & -0.259060000 \\
\hline & 6 & -3.016492000 & 0.146072000 & 0.056305000 \\
\hline & 8 & -3.034292000 & 1.355520000 & 0.182425000 \\
\hline & 8 & -4.113237000 & -0.609039000 & -0.017460000 \\
\hline
\end{tabular}




\begin{tabular}{|c|c|c|c|c|}
\hline & $\begin{array}{l}6 \\
6 \\
1 \\
1 \\
8 \\
6 \\
8 \\
8 \\
6 \\
6 \\
1 \\
1 \\
1 \\
6 \\
1 \\
1 \\
1 \\
6 \\
1 \\
1 \\
1 \\
6 \\
1 \\
1 \\
6 \\
1 \\
1 \\
1 \\
8 \\
1 \\
1\end{array}$ & $\begin{array}{c}-0.538020000 \\
-0.527684000 \\
-0.522587000 \\
0.618509000 \\
1.792201000 \\
1.927257000 \\
2.741830000 \\
4.166829000 \\
4.859815000 \\
5.947153000 \\
4.583156000 \\
4.586250000 \\
4.495405000 \\
4.173995000 \\
4.022308000 \\
5.582235000 \\
4.493951000 \\
4.188817000 \\
5.579004000 \\
4.004240000 \\
-5.368997000 \\
-5.410581000 \\
-5.406726000 \\
-6.460386000 \\
-7.440398000 \\
-6.403633000 \\
-6.385182000 \\
0.135570000 \\
0.781640000 \\
0.322835000\end{array}$ & $\begin{array}{r}0.227571000 \\
0.756889000 \\
1.006546000 \\
-0.599562000 \\
0.030830000 \\
1.232435000 \\
-0.878781000 \\
-0.500566000 \\
-1.845105000 \\
-1.703895000 \\
-2.495862000 \\
-2.345048000 \\
0.376108000 \\
-0.108751000 \\
1.359552000 \\
0.519031000 \\
0.161729000 \\
-0.481633000 \\
0.311311000 \\
1.135212000 \\
0.088649000 \\
0.834614000 \\
0.626859000 \\
-0.940041000 \\
-0.455589000 \\
-1.461322000 \\
-1.683567000 \\
3.469282000 \\
2.761784000 \\
3.726795000\end{array}$ & $\begin{array}{r}0.096744000 \\
1.060528000 \\
-0.677071000 \\
-0.010017000 \\
0.052173000 \\
0.168975000 \\
-0.033181000 \\
0.000197000 \\
-0.114322000 \\
-0.100196000 \\
0.724176000 \\
-1.051596000 \\
-1.193569000 \\
-2.124152000 \\
-1.120004000 \\
-1.242869000 \\
1.325342000 \\
2.160448000 \\
1.391217000 \\
1.426225000 \\
0.066829000 \\
-0.736553000 \\
1.022298000 \\
-0.051928000 \\
0.019709000 \\
-1.014464000 \\
0.749961000 \\
-0.160787000 \\
0.010082000 \\
-1.072101000\end{array}$ \\
\hline G & $\begin{array}{l}6 \\
6 \\
1 \\
1 \\
6 \\
8 \\
8 \\
6 \\
1 \\
1 \\
6 \\
1 \\
1 \\
1 \\
6 \\
1 \\
15 \\
6 \\
6 \\
6 \\
6 \\
1 \\
6 \\
1 \\
6 \\
1\end{array}$ & $\begin{array}{c}-1.393025000 \\
-1.899596000 \\
-2.054816000 \\
-0.324541000 \\
-3.399521000 \\
-4.139696000 \\
-3.868883000 \\
-5.296864000 \\
-5.720068000 \\
-5.681537000 \\
-5.610971000 \\
-6.695864000 \\
-5.176353000 \\
-5.216005000 \\
-1.183068000 \\
-1.694350000 \\
0.483838000 \\
1.610038000 \\
2.975962000 \\
1.059189000 \\
3.778396000 \\
3.415315000 \\
1.862905000 \\
-0.008950000 \\
3.221357000 \\
4.840560000\end{array}$ & $\begin{array}{r}-2.592609000 \\
-1.352617000 \\
-3.430485000 \\
-2.797051000 \\
-1.293645000 \\
-2.248243000 \\
-0.053229000 \\
0.075053000 \\
-0.270653000 \\
-0.585772000 \\
1.522427000 \\
1.667721000 \\
1.848073000 \\
2.163335000 \\
-0.121281000 \\
0.752925000 \\
0.083196000 \\
-0.793948000 \\
-0.971869000 \\
-1.271969000 \\
-1.609113000 \\
-0.611699000 \\
-1.913612000 \\
-1.138301000 \\
-2.080112000 \\
-1.742282000\end{array}$ & $\begin{array}{r}-0.051367000 \\
0.163628000 \\
-0.247943000 \\
-0.034804000 \\
0.190710000 \\
0.053962000 \\
0.394419000 \\
0.450268000 \\
-0.501857000 \\
1.237757000 \\
0.718081000 \\
0.768877000 \\
1.670460000 \\
-0.078872000 \\
0.346424000 \\
0.734837000 \\
0.061269000 \\
1.203808000 \\
0.954365000 \\
2.394195000 \\
1.893734000 \\
0.023438000 \\
3.331089000 \\
2.570099000 \\
3.081157000 \\
1.698059000\end{array}$ \\
\hline
\end{tabular}




\begin{tabular}{|c|c|c|c|c|}
\hline & $\begin{array}{l}1 \\
1 \\
6 \\
6 \\
6 \\
6 \\
1 \\
6 \\
1 \\
6 \\
1 \\
1 \\
1 \\
6 \\
6 \\
6 \\
6 \\
1 \\
6 \\
1 \\
6 \\
1 \\
1 \\
1\end{array}$ & $\begin{array}{c}1.427863000 \\
3.851260000 \\
1.029075000 \\
0.835451000 \\
1.529918000 \\
1.147007000 \\
0.444381000 \\
1.833477000 \\
1.702811000 \\
1.644435000 \\
1.003447000 \\
2.222404000 \\
1.887439000 \\
0.844502000 \\
2.059200000 \\
-0.099881000 \\
2.325176000 \\
2.798847000 \\
0.168959000 \\
-1.049988000 \\
1.382352000 \\
3.272455000 \\
-0.572213000 \\
1.592117000\end{array}$ & $\begin{array}{r}-2.287708000 \\
-2.582763000 \\
-0.431108000 \\
0.455523000 \\
-1.710155000 \\
0.075041000 \\
1.455530000 \\
-2.092786000 \\
-2.410606000 \\
-1.201612000 \\
0.778475000 \\
-3.090962000 \\
-1.500006000 \\
1.857397000 \\
2.302585000 \\
2.796410000 \\
3.664597000 \\
1.587157000 \\
4.156325000 \\
2.458090000 \\
4.591856000 \\
4.001370000 \\
4.877672000 \\
5.657087000\end{array}$ & $\begin{array}{c}4.255811000 \\
3.812654000 \\
-1.608846000 \\
-2.673355000 \\
-1.869827000 \\
-3.972538000 \\
-2.485985000 \\
-3.173049000 \\
-1.054037000 \\
-4.223809000 \\
-4.790226000 \\
-3.364362000 \\
-5.241856000 \\
0.231843000 \\
0.759927000 \\
-0.201698000 \\
0.853414000 \\
1.115124000 \\
-0.103864000 \\
-0.612958000 \\
0.421650000 \\
1.269584000 \\
-0.441868000 \\
0.496349000\end{array}$ \\
\hline complex $\mathbf{1 a}+\mathbf{G}$ & $\begin{array}{l}6 \\
6 \\
6 \\
6 \\
6 \\
6 \\
6 \\
6 \\
1 \\
1 \\
1 \\
8 \\
16 \\
6 \\
1 \\
17 \\
6 \\
6 \\
6 \\
6 \\
6 \\
1 \\
6 \\
1 \\
1 \\
1 \\
7 \\
1 \\
16 \\
8 \\
8 \\
6 \\
6 \\
\end{array}$ & $\begin{array}{c}3.881186000 \\
2.993276000 \\
2.873295000 \\
3.671279000 \\
4.574535000 \\
4.685806000 \\
2.571403000 \\
2.236488000 \\
2.165806000 \\
5.189062000 \\
5.387566000 \\
1.462456000 \\
3.847225000 \\
1.841194000 \\
1.075927000 \\
3.548043000 \\
1.881035000 \\
0.814668000 \\
2.963571000 \\
0.874986000 \\
3.014287000 \\
3.806155000 \\
1.971570000 \\
0.045423000 \\
3.873911000 \\
2.007219000 \\
-0.335689000 \\
-0.667831000 \\
-0.454056000 \\
-0.444007000 \\
-1.596636000 \\
1.020793000 \\
1.080973000 \\
\end{array}$ & $\begin{array}{c}2.208654000 \\
2.415077000 \\
3.659344000 \\
4.687334000 \\
4.491000000 \\
3.244480000 \\
0.097075000 \\
1.212872000 \\
3.800826000 \\
5.321182000 \\
3.095677000 \\
1.121108000 \\
0.555996000 \\
-1.033237000 \\
-0.960348000 \\
6.276572000 \\
-2.266983000 \\
-3.192016000 \\
-2.622839000 \\
-4.427105000 \\
-3.853477000 \\
-1.941684000 \\
-4.763475000 \\
-5.118999000 \\
-4.108188000 \\
-5.732626000 \\
-2.895722000 \\
-1.912139000 \\
-3.586578000 \\
-5.034374000 \\
-2.930309000 \\
-3.132286000 \\
-1.890970000 \\
\end{array}$ & $\begin{array}{c}1.065313000 \\
0.010587000 \\
-0.598632000 \\
-0.125575000 \\
0.922019000 \\
1.527732000 \\
0.570086000 \\
-0.362644000 \\
-1.414624000 \\
1.262613000 \\
2.345276000 \\
-1.301002000 \\
1.708815000 \\
0.500286000 \\
-0.274106000 \\
-0.852832000 \\
1.252103000 \\
1.122588000 \\
2.073487000 \\
1.763162000 \\
2.709770000 \\
2.177144000 \\
2.547276000 \\
1.635899000 \\
3.325561000 \\
3.039957000 \\
0.337317000 \\
0.339419000 \\
-1.189184000 \\
-1.017975000 \\
-1.816586000 \\
-2.054874000 \\
-2.685653000 \\
\end{array}$ \\
\hline
\end{tabular}




\begin{tabular}{|c|c|c|c|c|}
\hline & 6 & 2.137226000 & -3.961331000 & -1.987362000 \\
\hline & 6 & 2.271588000 & -1.489715000 & -3.270647000 \\
\hline & 1 & 0.213126000 & -1.232155000 & -2.697272000 \\
\hline & 6 & 3.323760000 & -3.537874000 & -2.572215000 \\
\hline & 1 & 2.074443000 & -4.923322000 & -1.482518000 \\
\hline & 6 & 3.408811000 & -2.303505000 & -3.221911000 \\
\hline & 1 & 2.324789000 & -0.517563000 & -3.761413000 \\
\hline & 1 & 4.204315000 & -4.177556000 & -2.524926000 \\
\hline & 6 & 4.684404000 & -1.859978000 & -3.868522000 \\
\hline & 1 & 4.892016000 & -0.803421000 & -3.658918000 \\
\hline & 1 & 5.539189000 & -2.454191000 & -3.526736000 \\
\hline & 1 & 4.625376000 & -1.962999000 & -4.960689000 \\
\hline & 6 & -0.086851000 & 1.835206000 & 1.680031000 \\
\hline & 6 & -0.674660000 & 0.627208000 & 1.837470000 \\
\hline & 1 & 0.659860000 & 2.173909000 & 2.393005000 \\
\hline & 1 & -0.317540000 & 2.495669000 & 0.845413000 \\
\hline & 6 & -0.201577000 & -0.145124000 & 3.036834000 \\
\hline & 8 & 0.644677000 & 0.227102000 & 3.824804000 \\
\hline & 8 & -0.830065000 & -1.323367000 & 3.165296000 \\
\hline & 6 & -0.445338000 & -2.141764000 & 4.278784000 \\
\hline & 1 & 0.607202000 & -2.432290000 & 4.151907000 \\
\hline & 1 & -0.515775000 & -1.548961000 & 5.199222000 \\
\hline & 6 & -1.363870000 & -3.333817000 & 4.290114000 \\
\hline & 1 & -1.074617000 & -4.026547000 & 5.088508000 \\
\hline & 1 & -2.403253000 & -3.027924000 & 4.457840000 \\
\hline & 1 & -1.310252000 & -3.868005000 & 3.332723000 \\
\hline & 6 & -1.677197000 & -0.009541000 & 1.006196000 \\
\hline & 1 & -2.225307000 & -0.828723000 & 1.469074000 \\
\hline & 15 & -2.693913000 & 0.898273000 & -0.052356000 \\
\hline & 6 & -3.527108000 & 2.333666000 & 0.720354000 \\
\hline & 6 & -4.099761000 & 3.382302000 & -0.009280000 \\
\hline & 6 & -3.656351000 & 2.319301000 & 2.111554000 \\
\hline & 6 & -4.793363000 & 4.394104000 & 0.645264000 \\
\hline & 1 & -4.002687000 & 3.420914000 & -1.093811000 \\
\hline & 6 & -4.348348000 & 3.333565000 & 2.764418000 \\
\hline & 1 & -3.206461000 & 1.505487000 & 2.679961000 \\
\hline & 6 & -4.916924000 & 4.370923000 & 2.031780000 \\
\hline & 1 & -5.236909000 & 5.203919000 & 0.069613000 \\
\hline & 1 & -4.440421000 & 3.314704000 & 3.848415000 \\
\hline & 1 & -5.456118000 & 5.166468000 & 2.542480000 \\
\hline & 6 & -1.869408000 & 1.534936000 & -1.545315000 \\
\hline & 6 & -1.652069000 & 0.636937000 & -2.595282000 \\
\hline & 6 & -1.392290000 & 2.843691000 & -1.651457000 \\
\hline & 6 & -0.971917000 & 1.045398000 & -3.736310000 \\
\hline & 1 & -2.019200000 & -0.388993000 & -2.517623000 \\
\hline & 6 & -0.718075000 & 3.250844000 & -2.798436000 \\
\hline & 1 & -1.549695000 & 3.558307000 & -0.844250000 \\
\hline & 6 & -0.506265000 & 2.353566000 & -3.839636000 \\
\hline & 1 & -0.811452000 & 0.340096000 & -4.550685000 \\
\hline & 1 & -0.357978000 & 4.275197000 & -2.876308000 \\
\hline & 1 & 0.020391000 & 2.674104000 & -4.736535000 \\
\hline & 6 & -4.033555000 & -0.185254000 & -0.630558000 \\
\hline & 6 & -5.132400000 & 0.381890000 & -1.286100000 \\
\hline & 6 & -3.978247000 & -1.569548000 & -0.458495000 \\
\hline & 6 & -6.159837000 & -0.423992000 & -1.758437000 \\
\hline & 1 & -5.194811000 & 1.460364000 & -1.424967000 \\
\hline & 6 & -5.010737000 & -2.373622000 & -0.931089000 \\
\hline
\end{tabular}




\begin{tabular}{|c|c|c|c|c|}
\hline & $\begin{array}{l}1 \\
6 \\
1 \\
1 \\
1\end{array}$ & $\begin{array}{l}-3.117748000 \\
-6.100720000 \\
-7.009726000 \\
-4.954044000 \\
-6.906188000\end{array}$ & $\begin{array}{r}-2.033380000 \\
-1.804029000 \\
0.027935000 \\
-3.451893000 \\
-2.435726000\end{array}$ & $\begin{array}{r}0.016748000 \\
-1.579394000 \\
-2.265627000 \\
-0.795827000 \\
-1.948951000\end{array}$ \\
\hline TS-1 & $\begin{array}{l}6 \\
6 \\
6 \\
6 \\
6 \\
6 \\
6 \\
6 \\
1 \\
1 \\
1 \\
8 \\
16 \\
6 \\
1 \\
17 \\
6 \\
6 \\
6 \\
6 \\
6 \\
1 \\
6 \\
1 \\
1 \\
1 \\
7 \\
1 \\
16 \\
8 \\
8 \\
6 \\
6 \\
6 \\
6 \\
1 \\
6 \\
1 \\
6 \\
1 \\
1 \\
6 \\
1 \\
1 \\
1 \\
6 \\
6 \\
1 \\
1 \\
6 \\
8 \\
8 \\
\end{array}$ & $\begin{array}{c}-4.635255000 \\
-4.100003000 \\
-4.646815000 \\
-5.741324000 \\
-6.289708000 \\
-5.737862000 \\
-2.630941000 \\
-2.946490000 \\
-4.210182000 \\
-7.151457000 \\
-6.165531000 \\
-2.329623000 \\
-3.781539000 \\
-1.512072000 \\
-0.947104000 \\
-6.465850000 \\
-0.922794000 \\
0.404408000 \\
-1.615926000 \\
0.967362000 \\
-1.044565000 \\
-2.639733000 \\
0.249900000 \\
1.982460000 \\
-1.606671000 \\
0.706705000 \\
1.149973000 \\
1.402896000 \\
1.611319000 \\
2.333985000 \\
2.286727000 \\
0.088446000 \\
-0.691057000 \\
-0.361961000 \\
-1.924528000 \\
-0.342488000 \\
-1.599526000 \\
0.254960000 \\
-2.394817000 \\
-2.540977000 \\
-1.958653000 \\
-3.717615000 \\
-4.464848000 \\
-4.104957000 \\
-3.631054000 \\
-0.368530000 \\
-0.43015000 \\
-0.43163498380000 \\
1.560307000 \\
\end{array}$ & $\begin{array}{c}-0.863509000 \\
-1.233528000 \\
-2.279966000 \\
-2.939555000 \\
-2.576014000 \\
-1.531510000 \\
0.571492000 \\
-0.421518000 \\
-2.556344000 \\
-3.117037000 \\
-1.250275000 \\
-0.545190000 \\
0.483535000 \\
1.316564000 \\
1.074086000 \\
-4.264215000 \\
2.328083000 \\
2.752274000 \\
2.947163000 \\
3.789709000 \\
3.973693000 \\
2.650859000 \\
4.401796000 \\
4.101269000 \\
4.447518000 \\
5.207149000 \\
2.096049000 \\
0.914014000 \\
2.904116000 \\
4.142038000 \\
1.921588000 \\
3.380576000 \\
2.391191000 \\
4.693256000 \\
2.727538000 \\
1.358846000 \\
5.016693000 \\
5.448647000 \\
4.045248000 \\
1.951766000 \\
6.044279000 \\
4.401958000 \\
3.618622000 \\
5.344945000 \\
4.521423000 \\
-1.731102000 \\
-0.866964000 \\
-1.901338000 \\
-2.263945000 \\
-0.189275000 \\
-0.330450000 \\
0.612267000 \\
\end{array}$ & $\begin{array}{c}1.305263000 \\
0.071410000 \\
-0.665705000 \\
-0.135534000 \\
1.097924000 \\
1.829155000 \\
0.724006000 \\
-0.337055000 \\
-1.623969000 \\
1.482004000 \\
2.788894000 \\
-1.386373000 \\
2.073120000 \\
0.608004000 \\
-0.294494000 \\
-1.025024000 \\
1.451450000 \\
1.172580000 \\
2.507416000 \\
1.920911000 \\
3.240113000 \\
2.728724000 \\
2.938963000 \\
1.683964000 \\
4.041825000 \\
3.511625000 \\
0.174995000 \\
0.294060000 \\
-1.155876000 \\
-0.844132000 \\
-2.009726000 \\
-1.940649000 \\
-2.537616000 \\
-1.865888000 \\
-3.072731000 \\
-2.564252000 \\
-2.413117000 \\
-1.383464000 \\
-3.024041000 \\
-3.527560000 \\
-2.360872000 \\
-3.629824000 \\
-3.453316000 \\
-3.227139000 \\
-4.718687000 \\
1.464394000 \\
1.742945000 \\
2.188242000 \\
0.519161000 \\
3.078629000 \\
3.858873000 \\
3.328890000 \\
\end{array}$ \\
\hline
\end{tabular}




\begin{tabular}{|c|c|c|c|c|}
\hline & 6 & 1.500164000 & 1.368217000 & 4.550054000 \\
\hline & 1 & 0.607768000 & 2.009867000 & 4.513248000 \\
\hline & 1 & 1.382150000 & 0.673702000 & 5.390981000 \\
\hline & 6 & 2.769357000 & 2.170655000 & 4.643251000 \\
\hline & 1 & 2.762457000 & 2.780045000 & 5.554147000 \\
\hline & 1 & 3.648560000 & 1.516282000 & 4.673578000 \\
\hline & 1 & 2.865054000 & 2.842249000 & 3.780886000 \\
\hline & 6 & 1.741310000 & -0.449796000 & 0.871338000 \\
\hline & 1 & 2.588333000 & -0.082002000 & 1.459347000 \\
\hline & 15 & 2.383064000 & -1.601903000 & -0.288496000 \\
\hline & 6 & 2.930203000 & -3.163363000 & 0.476988000 \\
\hline & 6 & 3.298298000 & -4.274744000 & -0.290653000 \\
\hline & 6 & 3.078498000 & -3.204079000 & 1.865468000 \\
\hline & 6 & 3.797815000 & -5.413193000 & 0.329150000 \\
\hline & 1 & 3.190968000 & -4.261600000 & -1.374861000 \\
\hline & 6 & 3.580521000 & -4.345405000 & 2.481585000 \\
\hline & 1 & 2.802365000 & -2.338164000 & 2.466870000 \\
\hline & 6 & 3.937631000 & -5.449394000 & 1.714558000 \\
\hline & 1 & 4.078538000 & -6.275549000 & -0.271825000 \\
\hline & 1 & 3.690056000 & -4.371317000 & 3.563557000 \\
\hline & 1 & 4.328463000 & -6.343346000 & 2.196462000 \\
\hline & 6 & 1.226560000 & -2.012171000 & -1.622236000 \\
\hline & 6 & 1.013001000 & -1.058596000 & -2.624276000 \\
\hline & 6 & 0.512036000 & -3.213596000 & -1.642061000 \\
\hline & 6 & 0.094468000 & -1.313395000 & -3.635990000 \\
\hline & 1 & 1.569642000 & -0.119757000 & -2.611120000 \\
\hline & 6 & -0.410568000 & -3.457258000 & -2.653683000 \\
\hline & 1 & 0.666560000 & -3.962584000 & -0.866152000 \\
\hline & 6 & -0.616851000 & -2.509645000 & -3.651115000 \\
\hline & 1 & -0.065846000 & -0.571247000 & -4.416505000 \\
\hline & 1 & -0.964760000 & -4.393372000 & -2.663230000 \\
\hline & 1 & -1.337009000 & -2.703311000 & -4.443666000 \\
\hline & 6 & 3.857235000 & -0.892448000 & -1.070965000 \\
\hline & 6 & 4.323903000 & -1.450033000 & -2.265982000 \\
\hline & 6 & 4.567820000 & 0.151364000 & -0.474500000 \\
\hline & 6 & 5.487219000 & -0.970307000 & -2.854268000 \\
\hline & 1 & 3.768227000 & -2.249123000 & -2.755754000 \\
\hline & 6 & 5.732379000 & 0.627439000 & -1.066205000 \\
\hline & 1 & 4.215621000 & 0.613490000 & 0.445335000 \\
\hline & 6 & 6.193383000 & 0.067908000 & -2.253444000 \\
\hline & 1 & 5.839826000 & -1.405210000 & -3.787047000 \\
\hline & 1 & 6.276902000 & 1.444039000 & -0.597030000 \\
\hline & 1 & 7.104238000 & 0.444891000 & -2.714296000 \\
\hline & 6 & -4.562195000 & -1.228389000 & 0.712555000 \\
\hline & 6 & -3.718644000 & -1.594067000 & -0.336997000 \\
\hline & 6 & -3.983121000 & -2.715611000 & -1.117213000 \\
\hline & 6 & -5.112763000 & -3.457216000 & -0.819858000 \\
\hline & 6 & -5.968757000 & -3.099902000 & 0.226310000 \\
\hline & 6 & -5.698634000 & -1.978871000 & 1.001094000 \\
\hline INT_1 & 6 & -2.580156000 & 0.341067000 & 0.543366000 \\
\hline $1 \sqrt{1} 1-1$ & 6 & -2.567314000 & -0.699643000 & -0.511262000 \\
\hline & 1 & -3.308112000 & -2.981879000 & -1.929081000 \\
\hline & 1 & -6.847181000 & -3.706860000 & 0.432764000 \\
\hline & 1 & -6.364503000 & -1.704888000 & 1.816484000 \\
\hline & 8 & -1.718411000 & -0.810672000 & -1.389305000 \\
\hline & 16 & -4.027923000 & 0.222927000 & 1.568936000 \\
\hline & 6 & -1.525543000 & 1.183312000 & 0.657957000 \\
\hline
\end{tabular}




\begin{tabular}{|c|c|c|c|c|}
\hline & 1 & -0.747612000 & 0.989291000 & -0.085006000 \\
\hline & 17 & -5.489293000 & -4.881078000 & -1.770248000 \\
\hline & 6 & -1.219308000 & 2.272441000 & 1.545911000 \\
\hline & 6 & 0.062309000 & 2.894421000 & 1.407784000 \\
\hline & 6 & -2.130133000 & 2.776981000 & 2.494348000 \\
\hline & 6 & 0.340753000 & 4.016215000 & 2.211872000 \\
\hline & 6 & -1.824736000 & 3.872381000 & 3.280790000 \\
\hline & 1 & -3.111685000 & 2.316437000 & 2.600151000 \\
\hline & 6 & -0.582741000 & 4.495499000 & 3.126783000 \\
\hline & 1 & 1.310530000 & 4.495591000 & 2.101371000 \\
\hline & 1 & -2.546927000 & 4.248963000 & 4.001939000 \\
\hline & 1 & -0.331239000 & 5.361894000 & 3.737437000 \\
\hline & 7 & 0.984007000 & 2.351135000 & 0.521659000 \\
\hline & 1 & 1.235856000 & 0.126146000 & 0.841688000 \\
\hline & 16 & 1.543684000 & 3.285474000 & -0.634420000 \\
\hline & 8 & 2.149753000 & 4.563965000 & -0.204703000 \\
\hline & 8 & 2.396156000 & 2.458930000 & -1.510188000 \\
\hline & 6 & 0.096411000 & 3.731285000 & -1.591093000 \\
\hline & 6 & -0.606399000 & 2.721793000 & -2.248640000 \\
\hline & 6 & -0.376825000 & 5.036584000 & -1.606602000 \\
\hline & 6 & -1.773934000 & 3.027316000 & -2.930897000 \\
\hline & 1 & -0.250183000 & 1.692337000 & -2.211261000 \\
\hline & 6 & -1.548580000 & 5.333215000 & -2.298851000 \\
\hline & 1 & 0.177238000 & 5.813344000 & -1.083338000 \\
\hline & 6 & -2.263873000 & 4.338669000 & -2.966731000 \\
\hline & 1 & -2.320458000 & 2.234211000 & -3.443232000 \\
\hline & 1 & -1.916634000 & 6.359226000 & -2.319287000 \\
\hline & 6 & -3.539732000 & 4.655420000 & -3.686826000 \\
\hline & 1 & -4.414985000 & 4.390000000 & -3.077575000 \\
\hline & 1 & -3.615778000 & 5.723852000 & -3.920127000 \\
\hline & 1 & -3.621364000 & 4.092599000 & -4.624917000 \\
\hline & 6 & -0.290383000 & -2.717532000 & 0.755508000 \\
\hline & 6 & 0.224333000 & -1.701328000 & 1.449645000 \\
\hline & 1 & -1.210081000 & -3.185011000 & 1.100278000 \\
\hline & 1 & 0.149359000 & -3.114318000 & -0.154809000 \\
\hline & 6 & -0.536921000 & -1.280268000 & 2.667953000 \\
\hline & 8 & -1.573475000 & -1.790344000 & 3.037811000 \\
\hline & 8 & 0.070219000 & -0.279294000 & 3.309436000 \\
\hline & 6 & -0.609554000 & 0.241305000 & 4.466886000 \\
\hline & 1 & -1.582438000 & 0.635782000 & 4.140293000 \\
\hline & 1 & -0.793992000 & -0.582563000 & 5.167138000 \\
\hline & 6 & 0.262928000 & 1.317703000 & 5.051483000 \\
\hline & 1 & -0.228693000 & 1.763921000 & 5.923527000 \\
\hline & 1 & 1.229216000 & 0.910052000 & 5.371593000 \\
\hline & 1 & 0.440744000 & 2.111272000 & 4.314389000 \\
\hline & 6 & 1.471816000 & -0.917683000 & 1.140569000 \\
\hline & 1 & 2.071192000 & -0.816149000 & 2.057320000 \\
\hline & 15 & 2.615726000 & -1.520501000 & -0.132822000 \\
\hline & 6 & 3.331987000 & -3.101423000 & 0.359950000 \\
\hline & 6 & 4.269136000 & -3.710911000 & -0.484325000 \\
\hline & 6 & 3.015639000 & -3.685943000 & 1.589393000 \\
\hline & 6 & 4.873786000 & -4.899757000 & -0.101982000 \\
\hline & 1 & 4.526880000 & -3.260915000 & -1.442517000 \\
\hline & 6 & 3.629135000 & -4.875396000 & 1.966419000 \\
\hline & 1 & 2.288114000 & -3.226883000 & 2.255385000 \\
\hline & 6 & 4.553924000 & -5.481217000 & 1.122998000 \\
\hline & 1 & 5.597911000 & -5.372181000 & -0.761379000 \\
\hline
\end{tabular}




\begin{tabular}{|c|c|c|c|c|}
\hline & $\begin{array}{l}1 \\
1 \\
6 \\
6 \\
6 \\
6 \\
1 \\
6 \\
1 \\
6 \\
1 \\
1 \\
1 \\
6 \\
6 \\
6 \\
6 \\
1 \\
6 \\
1 \\
6 \\
1 \\
1 \\
1\end{array}$ & $\begin{array}{l}3.380203000 \\
5.030951000 \\
1.822931000 \\
1.479617000 \\
1.522741000 \\
0.834574000 \\
1.732888000 \\
0.867567000 \\
1.798281000 \\
0.525539000 \\
0.572966000 \\
0.633677000 \\
0.019641000 \\
3.930670000 \\
4.709794000 \\
4.210244000 \\
5.766365000 \\
4.480426000 \\
5.266958000 \\
3.607891000 \\
6.044245000 \\
6.368728000 \\
5.478450000 \\
6.869699000\end{array}$ & $\begin{array}{r}-5.328771000 \\
-6.412382000 \\
-1.661220000 \\
-0.475305000 \\
-2.903920000 \\
-0.545513000 \\
0.499060000 \\
-2.958284000 \\
-3.828555000 \\
-1.780518000 \\
0.373045000 \\
-3.923326000 \\
-1.827159000 \\
-0.293031000 \\
-0.271435000 \\
0.589359000 \\
0.622544000 \\
-0.934942000 \\
1.484834000 \\
0.599167000 \\
1.499765000 \\
0.638598000 \\
2.175408000 \\
2.203016000\end{array}$ & $\begin{array}{c}2.922945000 \\
1.421035000 \\
-1.742639000 \\
-2.401442000 \\
-2.310066000 \\
-3.630662000 \\
-1.970632000 \\
-3.534106000 \\
-1.803721000 \\
-4.192812000 \\
-4.153717000 \\
-3.977686000 \\
-5.155031000 \\
-0.275300000 \\
-1.437276000 \\
0.771224000 \\
-1.544390000 \\
-2.270563000 \\
0.653808000 \\
1.676485000 \\
-0.499533000 \\
-2.449791000 \\
1.466917000 \\
-0.587819000\end{array}$ \\
\hline TS-2 & $\begin{array}{l}6 \\
6 \\
6 \\
6 \\
6 \\
6 \\
6 \\
6 \\
1 \\
1 \\
1 \\
8 \\
16 \\
6 \\
1 \\
17 \\
6 \\
6 \\
6 \\
6 \\
6 \\
1 \\
6 \\
1 \\
1 \\
1 \\
7 \\
1 \\
16 \\
8 \\
8 \\
6 \\
6 \\
6 \\
6 \\
6 \\
6 \\
6 \\
6\end{array}$ & $\begin{array}{c}-5.038638000 \\
-4.906148000 \\
-5.712911000 \\
-6.651200000 \\
-6.795414000 \\
-5.987508000 \\
-3.205651000 \\
-3.854429000 \\
-5.590268000 \\
-7.543941000 \\
-6.101791000 \\
-3.536934000 \\
-3.909684000 \\
-2.148005000 \\
-1.833442000 \\
-7.681647000 \\
-1.348783000 \\
-0.005012000 \\
-1.859419000 \\
0.771021000 \\
-1.081901000 \\
-2.900258000 \\
0.236365000 \\
1.807469000 \\
-1.498630000 \\
0.858981000 \\
0.469985000 \\
1.702354000 \\
1.554334000 \\
2.822682000 \\
1.684313000 \\
0.751457000 \\
-0.428205000 \\
\end{array}$ & $\begin{array}{c}-0.947250000 \\
-0.753655000 \\
-1.438000000 \\
-2.318978000 \\
-2.520320000 \\
-1.833831000 \\
0.723925000 \\
0.204749000 \\
-1.275955000 \\
-3.220237000 \\
-1.993247000 \\
0.512290000 \\
0.015314000 \\
1.558621000 \\
1.766653000 \\
-3.210194000 \\
2.165040000 \\
2.523169000 \\
2.436065000 \\
3.134486000 \\
3.049776000 \\
2.202487000 \\
3.396297000 \\
3.385413000 \\
3.262258000 \\
3.869570000 \\
2.131613000 \\
0.375859000 \\
3.068723000 \\
3.267254000 \\
2.498135000 \\
4.650576000 \\
4.740196000 \\
\end{array}$ & $\begin{array}{c}1.112088000 \\
-0.263558000 \\
-1.168350000 \\
-0.659957000 \\
0.716187000 \\
1.614270000 \\
0.604591000 \\
-0.625336000 \\
-2.237956000 \\
1.080844000 \\
2.684100000 \\
-1.767139000 \\
2.075468000 \\
0.517837000 \\
-0.507781000 \\
-1.759717000 \\
1.559823000 \\
1.254302000 \\
2.839263000 \\
2.253611000 \\
3.806613000 \\
3.061258000 \\
3.506261000 \\
2.036937000 \\
4.788547000 \\
4.263781000 \\
0.003038000 \\
-0.900623000 \\
-0.777178000 \\
-0.049502000 \\
-2.124975000 \\
-0.908503000 \\
-1.647497000 \\
\end{array}$ \\
\hline
\end{tabular}




\begin{tabular}{|c|c|c|c|c|}
\hline & 6 & 1.284005000 & 5.764646000 & -0.273098000 \\
\hline & 6 & -1.070064000 & 5.963881000 & -1.749251000 \\
\hline & 1 & -0.831158000 & 3.857492000 & -2.142419000 \\
\hline & 6 & 0.627473000 & 6.986290000 & -0.386678000 \\
\hline & 1 & 2.205212000 & 5.674343000 & 0.297993000 \\
\hline & 6 & -0.553864000 & 7.103870000 & -1.119332000 \\
\hline & 1 & -1.989838000 & 6.044646000 & -2.328403000 \\
\hline & 1 & 1.041377000 & 7.866894000 & 0.103898000 \\
\hline & 6 & -1.271288000 & 8.414610000 & -1.225693000 \\
\hline & 1 & -2.200126000 & 8.404133000 & -0.639259000 \\
\hline & 1 & -0.655596000 & 9.242929000 & -0.857631000 \\
\hline & 1 & -1.553532000 & 8.632157000 & -2.263508000 \\
\hline & 6 & 0.017477000 & -0.615200000 & 1.897335000 \\
\hline & 6 & 0.369538000 & -0.519704000 & 0.605758000 \\
\hline & 1 & -0.852330000 & -1.189086000 & 2.207574000 \\
\hline & 1 & 0.578836000 & -0.097520000 & 2.675675000 \\
\hline & 6 & -0.414687000 & -1.166561000 & -0.486101000 \\
\hline & 8 & -0.241854000 & -0.916691000 & -1.661505000 \\
\hline & 8 & -1.302609000 & -2.048781000 & -0.029294000 \\
\hline & 6 & -2.068543000 & -2.754220000 & -1.024472000 \\
\hline & 1 & -2.663071000 & -2.024322000 & -1.587519000 \\
\hline & 1 & -1.371199000 & -3.226312000 & -1.728338000 \\
\hline & 6 & -2.915386000 & -3.759461000 & -0.295414000 \\
\hline & 1 & -3.558766000 & -4.295874000 & -1.002366000 \\
\hline & 1 & -2.284635000 & -4.490356000 & 0.225953000 \\
\hline & 1 & -3.555742000 & -3.263828000 & 0.446428000 \\
\hline & 6 & 1.506230000 & 0.271009000 & 0.158913000 \\
\hline & 1 & 2.178853000 & 0.731014000 & 0.876764000 \\
\hline & 15 & 3.109989000 & -1.681183000 & -0.015932000 \\
\hline & 6 & 4.212750000 & -1.946744000 & 1.414080000 \\
\hline & 6 & 5.555481000 & -2.309017000 & 1.271343000 \\
\hline & 6 & 3.689274000 & -1.743962000 & 2.697007000 \\
\hline & 6 & 6.354456000 & -2.482267000 & 2.396369000 \\
\hline & 1 & 5.980811000 & -2.453879000 & 0.278963000 \\
\hline & 6 & 4.488525000 & -1.928728000 & 3.819908000 \\
\hline & 1 & 2.648092000 & -1.442343000 & 2.820790000 \\
\hline & 6 & 5.822426000 & -2.297045000 & 3.669400000 \\
\hline & 1 & 7.398597000 & -2.764912000 & 2.277086000 \\
\hline & 1 & 4.069750000 & -1.776134000 & 4.812518000 \\
\hline & 1 & 6.450875000 & -2.434933000 & 4.546946000 \\
\hline & 6 & 2.200923000 & -3.242299000 & -0.257148000 \\
\hline & 6 & 1.701379000 & -3.561342000 & -1.526521000 \\
\hline & 6 & 1.833273000 & -4.031192000 & 0.838300000 \\
\hline & 6 & 0.879360000 & -4.669130000 & -1.698478000 \\
\hline & 1 & 1.959352000 & -2.944095000 & -2.386658000 \\
\hline & 6 & 0.999279000 & -5.130579000 & 0.663302000 \\
\hline & 1 & 2.206235000 & -3.793726000 & 1.834366000 \\
\hline & 6 & 0.523717000 & -5.454211000 & -0.604145000 \\
\hline & 1 & 0.512624000 & -4.918040000 & -2.692885000 \\
\hline & 1 & 0.724939000 & -5.739947000 & 1.522323000 \\
\hline & 1 & -0.126012000 & -6.316844000 & -0.739982000 \\
\hline & 6 & 4.197574000 & -1.477517000 & -1.459023000 \\
\hline & 6 & 4.781786000 & -2.551157000 & -2.141815000 \\
\hline & 6 & 4.477942000 & -0.167967000 & -1.865357000 \\
\hline & 6 & 5.632170000 & -2.314118000 & -3.215181000 \\
\hline & 1 & 4.568077000 & -3.574167000 & -1.832027000 \\
\hline & 6 & 5.332932000 & 0.064704000 & -2.937876000 \\
\hline
\end{tabular}




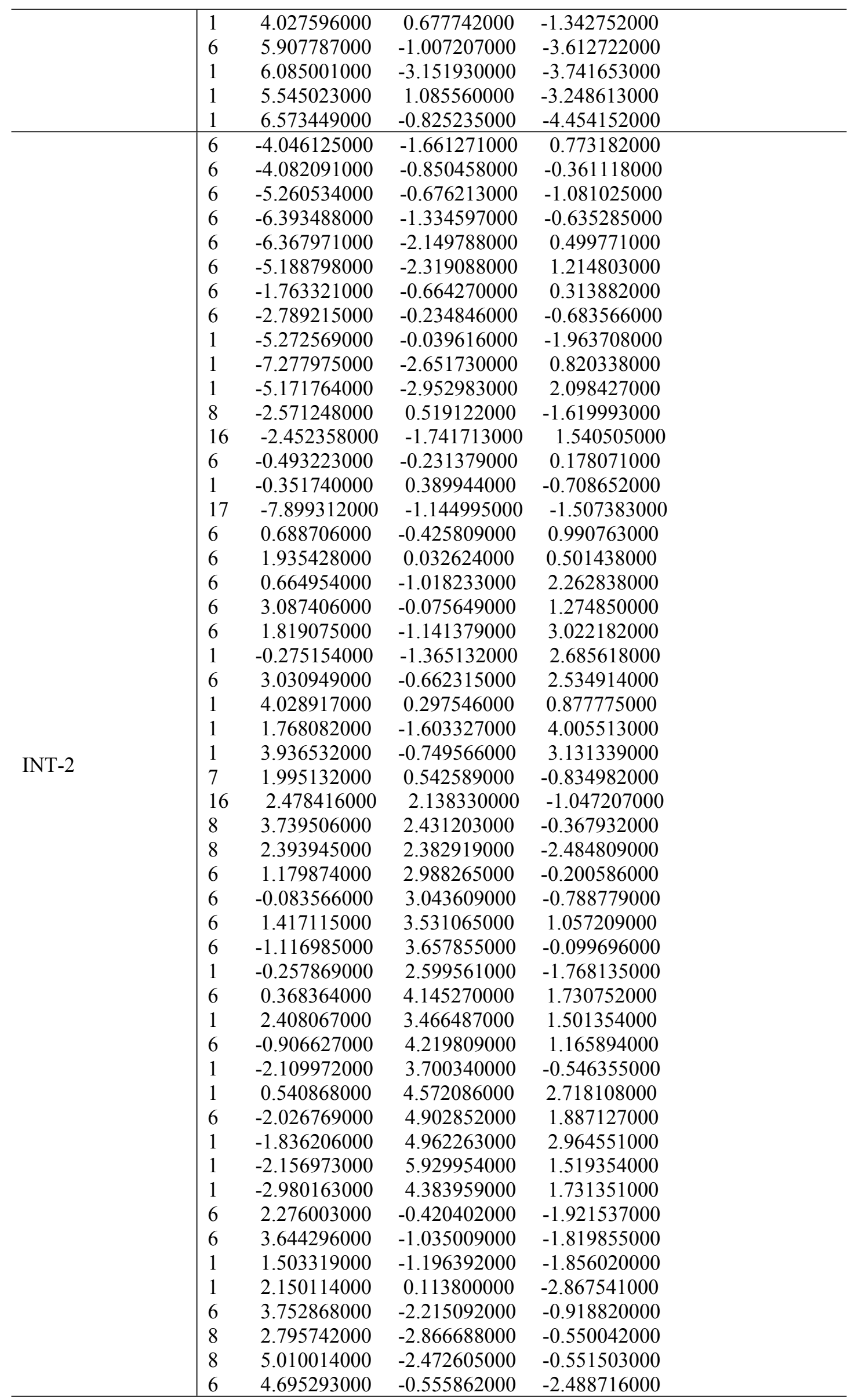




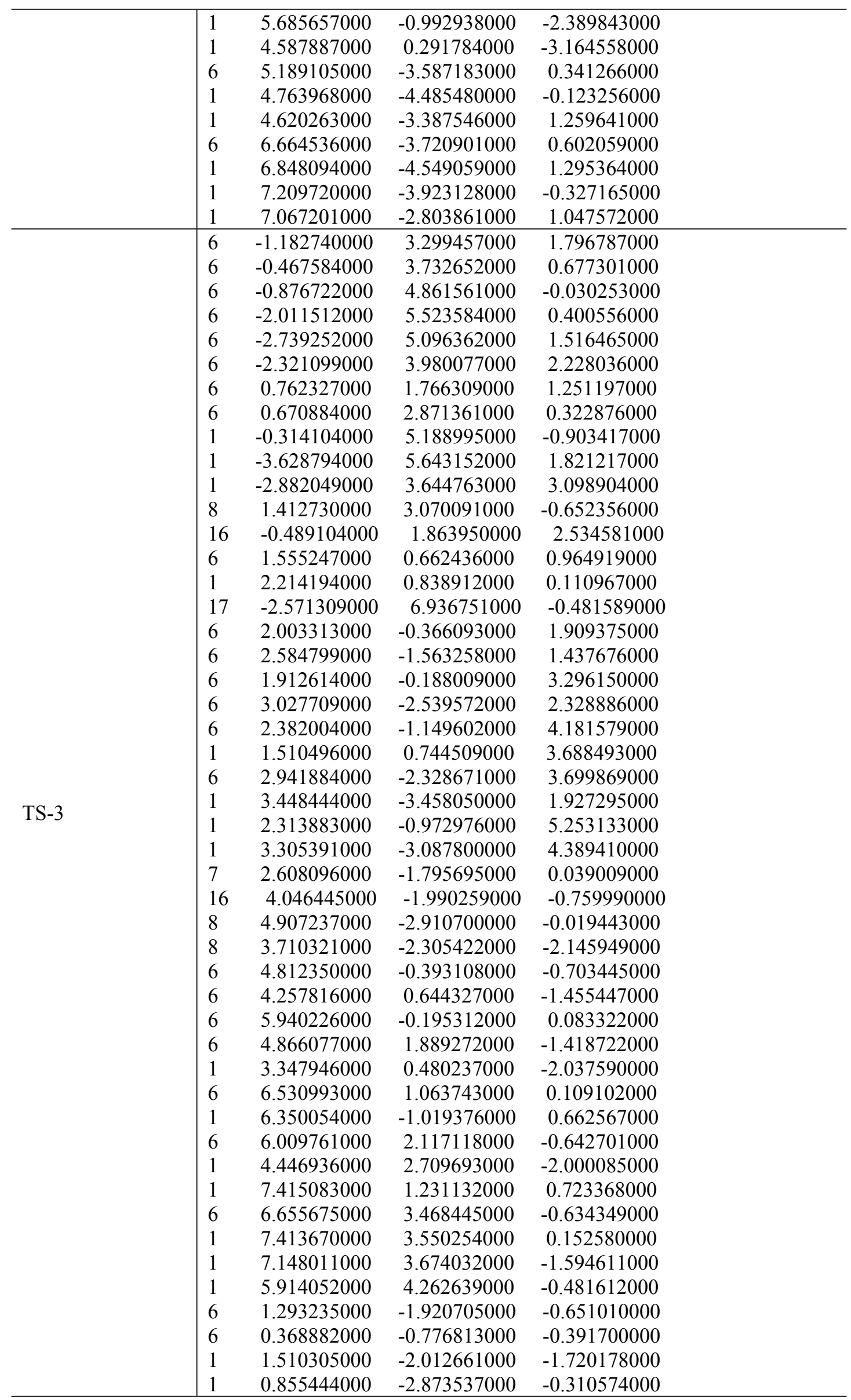




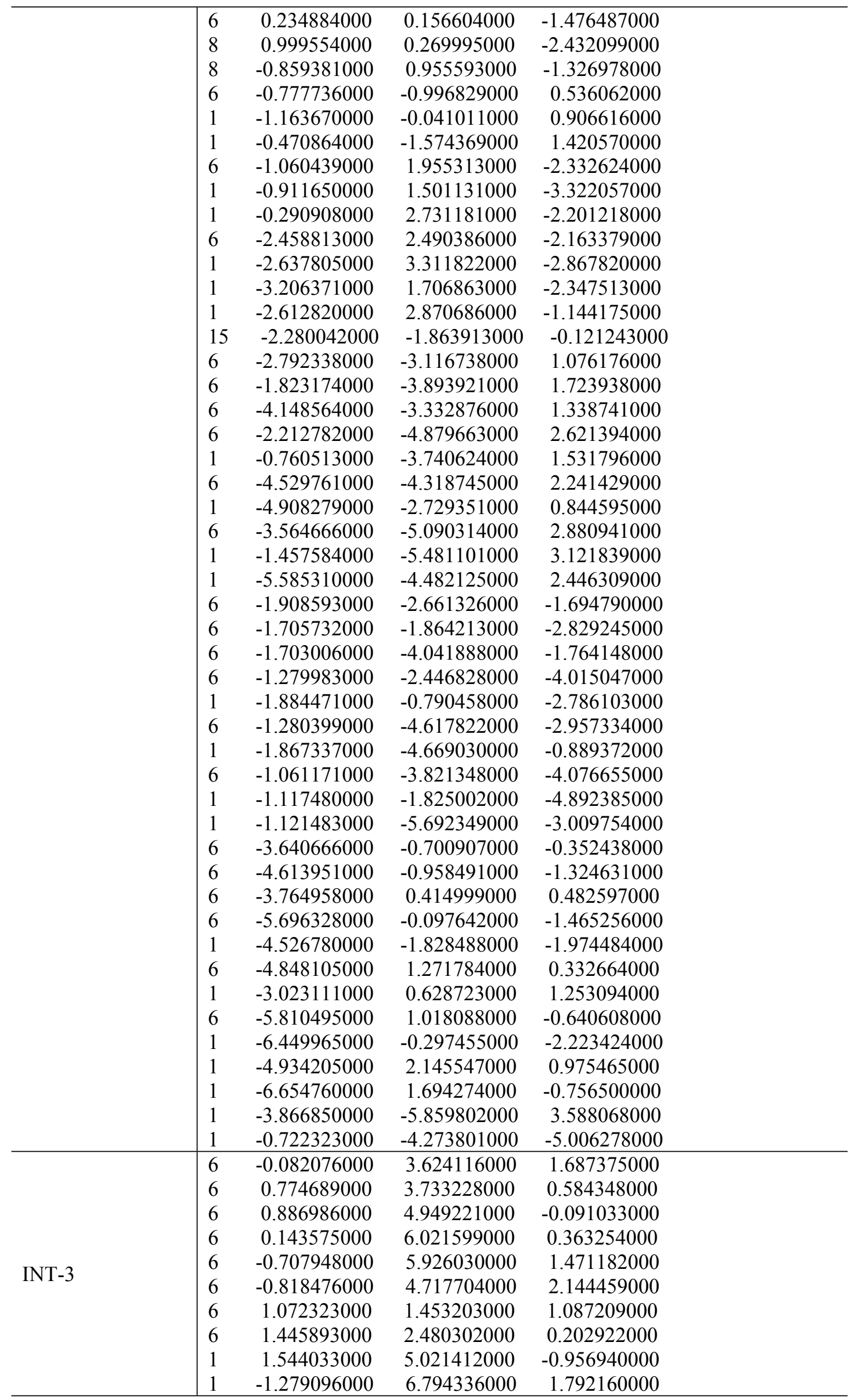




\begin{tabular}{|c|c|c|c|c|}
\hline & 1 & -1.479696000 & 4.630238000 & 3.005444000 \\
\hline & 8 & 2.190520000 & 2.385354000 & -0.816634000 \\
\hline & 16 & -0.073376000 & 2.004116000 & 2.348530000 \\
\hline & 6 & 1.327736000 & 0.027638000 & 0.737450000 \\
\hline & 1 & 2.163525000 & 0.101184000 & 0.019088000 \\
\hline & 17 & 0.260818000 & 7.569703000 & -0.473081000 \\
\hline & 6 & 1.772907000 & -0.918931000 & 1.824883000 \\
\hline & 6 & 2.056915000 & -2.242198000 & 1.451896000 \\
\hline & 6 & 1.979992000 & -0.554680000 & 3.152702000 \\
\hline & 6 & 2.520207000 & -3.176220000 & 2.374168000 \\
\hline & 6 & 2.457785000 & -1.478445000 & 4.079855000 \\
\hline & 1 & 1.796124000 & 0.473826000 & 3.457557000 \\
\hline & 6 & 2.729714000 & -2.786059000 & 3.693080000 \\
\hline & 1 & 2.724078000 & -4.192141000 & 2.045543000 \\
\hline & 1 & 2.621353000 & -1.169431000 & 5.110573000 \\
\hline & 1 & 3.101026000 & -3.508038000 & 4.417636000 \\
\hline & 7 & 1.829814000 & -2.581313000 & 0.087835000 \\
\hline & 16 & 3.119301000 & -3.052251000 & -0.874931000 \\
\hline & 8 & 3.737931000 & -4.217341000 & -0.251079000 \\
\hline & 8 & 2.590332000 & -3.164135000 & -2.230005000 \\
\hline & 6 & 4.294228000 & -1.729157000 & -0.811712000 \\
\hline & 6 & 4.027720000 & -0.557372000 & -1.519659000 \\
\hline & 6 & 5.436821000 & -1.861712000 & -0.029602000 \\
\hline & 6 & 4.932739000 & 0.488896000 & -1.439718000 \\
\hline & 1 & 3.111555000 & -0.457889000 & -2.102918000 \\
\hline & 6 & 6.337023000 & -0.804405000 & 0.026287000 \\
\hline & 1 & 5.614972000 & -2.783908000 & 0.519126000 \\
\hline & 6 & 6.100692000 & 0.379488000 & -0.675727000 \\
\hline & 1 & 4.718200000 & 1.419108000 & -1.963872000 \\
\hline & 1 & 7.237940000 & -0.896041000 & 0.631949000 \\
\hline & 6 & 7.071011000 & 1.518858000 & -0.619829000 \\
\hline & 1 & 7.845502000 & 1.355593000 & 0.137831000 \\
\hline & 1 & 7.572497000 & 1.655956000 & -1.587415000 \\
\hline & 1 & 6.560628000 & 2.463121000 & -0.391344000 \\
\hline & 6 & 0.568951000 & -2.130164000 & -0.492854000 \\
\hline & 6 & 0.174212000 & -0.675816000 & -0.133603000 \\
\hline & 1 & 0.630583000 & -2.253057000 & -1.578108000 \\
\hline & 1 & -0.205822000 & -2.823470000 & -0.134165000 \\
\hline & 6 & 0.103660000 & 0.149665000 & -1.409095000 \\
\hline & 8 & 0.788489000 & -0.058638000 & -2.387352000 \\
\hline & 8 & -0.782527000 & 1.138839000 & -1.310742000 \\
\hline & 6 & -1.118289000 & -0.646300000 & 0.692263000 \\
\hline & 1 & -1.308769000 & 0.378001000 & 1.026014000 \\
\hline & 1 & -0.962972000 & -1.235524000 & 1.606337000 \\
\hline & 6 & -0.758055000 & 2.137750000 & -2.352827000 \\
\hline & 1 & -0.910487000 & 1.645219000 & -3.322875000 \\
\hline & 1 & 0.246432000 & 2.578360000 & -2.340255000 \\
\hline & 6 & -1.840088000 & 3.132456000 & -2.034518000 \\
\hline & 1 & -1.808722000 & 3.961565000 & -2.750670000 \\
\hline & 1 & -2.834872000 & 2.669537000 & -2.086185000 \\
\hline & 1 & -1.697453000 & 3.540981000 & -1.024346000 \\
\hline & 15 & -2.715666000 & -1.195329000 & -0.020018000 \\
\hline & 6 & -3.498577000 & -2.292341000 & 1.183783000 \\
\hline & 6 & -2.734268000 & -3.190153000 & 1.939738000 \\
\hline & 6 & -4.891148000 & -2.281212000 & 1.313531000 \\
\hline & 6 & -3.362621000 & -4.065281000 & 2.816442000 \\
\hline & 1 & -1.648353000 & -3.221449000 & 1.852726000 \\
\hline
\end{tabular}




\begin{tabular}{|c|c|c|c|c|}
\hline & $\begin{array}{l}6 \\
1 \\
6 \\
1 \\
1 \\
6 \\
6 \\
6 \\
6 \\
1 \\
6 \\
1 \\
6 \\
1 \\
1 \\
6 \\
6 \\
6 \\
6 \\
1 \\
6 \\
1 \\
6 \\
1 \\
1 \\
1 \\
1 \\
1\end{array}$ & $\begin{array}{l}-5.510877000 \\
-5.494375000 \\
-4.748479000 \\
-2.765105000 \\
-6.593243000 \\
-2.518964000 \\
-2.279349000 \\
-2.511944000 \\
-2.006700000 \\
-2.315993000 \\
-2.238797000 \\
-2.715123000 \\
-1.977835000 \\
-1.815482000 \\
-2.230662000 \\
-3.790101000 \\
-4.790217000 \\
-3.690954000 \\
-5.674427000 \\
-4.881801000 \\
-4.577139000 \\
-2.924842000 \\
-5.566268000 \\
-6.448185000 \\
-4.488703000 \\
-6.258374000 \\
-5.235776000 \\
-1.758020000\end{array}$ & $\begin{array}{r}-3.158287000 \\
-1.584681000 \\
-4.047844000 \\
-4.758835000 \\
-3.141972000 \\
-2.079710000 \\
-1.362201000 \\
-3.478364000 \\
-2.042455000 \\
-0.274348000 \\
-4.150709000 \\
-4.044208000 \\
-3.434473000 \\
-1.482769000 \\
-5.237804000 \\
0.231181000 \\
0.174296000 \\
1.351061000 \\
1.236726000 \\
-0.696651000 \\
2.408950000 \\
1.418743000 \\
2.353454000 \\
1.189140000 \\
3.281794000 \\
3.183993000 \\
-4.731164000 \\
-3.965027000\end{array}$ & $\begin{array}{c}.195070000 \\
0.733490000 \\
2.945666000 \\
3.403086000 \\
2.297674000 \\
-1.580433000 \\
-2.758894000 \\
-1.597593000 \\
-3.937978000 \\
-2.754619000 \\
-2.782252000 \\
-0.689432000 \\
-3.946985000 \\
-4.850485000 \\
-2.794405000 \\
-0.280688000 \\
-1.258771000 \\
0.552527000 \\
-1.406383000 \\
-1.906716000 \\
0.395380000 \\
1.324629000 \\
-0.582487000 \\
-2.168885000 \\
1.038488000 \\
-0.702727000 \\
3.637597000 \\
-4.870851000\end{array}$ \\
\hline TS-4 & $\begin{array}{l}6 \\
6 \\
6 \\
6 \\
6 \\
6 \\
6 \\
6 \\
1 \\
1 \\
1 \\
8 \\
16 \\
6 \\
1 \\
17 \\
6 \\
6 \\
6 \\
6 \\
6 \\
1 \\
6 \\
1 \\
1 \\
1 \\
7 \\
16 \\
8\end{array}$ & $\begin{array}{c}3.951645000 \\
3.200842000 \\
3.760216000 \\
5.079745000 \\
5.845273000 \\
5.282682000 \\
1.627757000 \\
1.814727000 \\
3.159844000 \\
6.880022000 \\
5.874163000 \\
0.968763000 \\
3.038495000 \\
0.262393000 \\
0.436026000 \\
5.828428000 \\
-0.661142000 \\
-1.871717000 \\
-0.283741000 \\
-2.661478000 \\
-1.074499000 \\
0.662719000 \\
-2.272383000 \\
-3.585133000 \\
-0.755965000 \\
-2.899773000 \\
-2.262089000 \\
-3.846982000 \\
-3.774484000 \\
\end{array}$ & $\begin{array}{l}-2.246496000 \\
-2.350383000 \\
-2.894874000 \\
-3.304204000 \\
-3.197234000 \\
-2.665787000 \\
-1.212577000 \\
-1.866699000 \\
-2.978783000 \\
-3.531887000 \\
-2.584795000 \\
-1.947827000 \\
-1.557770000 \\
-1.097944000 \\
-1.101762000 \\
-3.969328000 \\
-2.255530000 \\
-2.116866000 \\
-3.534380000 \\
-3.236143000 \\
-4.647022000 \\
-3.645391000 \\
-4.493383000 \\
-3.116230000 \\
-5.630103000 \\
-5.355616000 \\
-0.825291000 \\
-0.293778000 \\
1.158685000\end{array}$ & $\begin{array}{c}-0.965159000 \\
0.210131000 \\
1.364678000 \\
1.318226000 \\
0.151820000 \\
-0.999045000 \\
-1.202722000 \\
0.087272000 \\
2.269193000 \\
0.154334000 \\
-1.908873000 \\
0.985635000 \\
-2.298284000 \\
-1.868998000 \\
-2.956624000 \\
2.762054000 \\
-1.566343000 \\
-0.876578000 \\
-1.980529000 \\
-0.601539000 \\
-1.732026000 \\
-2.512742000 \\
-1.038028000 \\
-0.039000000 \\
-2.073032000 \\
-0.820820000 \\
-0.429512000 \\
-0.611647000 \\
-0.760921000\end{array}$ \\
\hline
\end{tabular}




\begin{tabular}{|c|c|c|c|c|}
\hline & 8 & -4.518559000 & -1.089615000 & -1.630839000 \\
\hline & 6 & -4.610365000 & -0.650053000 & 0.949609000 \\
\hline & 6 & -5.747269000 & -1.447933000 & 0.992561000 \\
\hline & 6 & -4.077113000 & -0.088841000 & 2.109616000 \\
\hline & 6 & -6.352894000 & -1.690756000 & 2.220718000 \\
\hline & 1 & -6.148665000 & -1.870828000 & 0.074195000 \\
\hline & 6 & -4.693632000 & -0.343188000 & 3.323343000 \\
\hline & 1 & -3.189047000 & 0.540615000 & 2.069384000 \\
\hline & 6 & -5.836793000 & -1.149376000 & 3.398536000 \\
\hline & 1 & -7.245317000 & -2.313975000 & 2.263724000 \\
\hline & 1 & -4.284685000 & 0.088687000 & 4.236340000 \\
\hline & 6 & -6.473625000 & -1.435315000 & 4.723195000 \\
\hline & 1 & -6.515137000 & -0.536189000 & 5.350003000 \\
\hline & 1 & -7.491744000 & -1.822868000 & 4.606254000 \\
\hline & 1 & -5.896492000 & -2.186315000 & 5.279862000 \\
\hline & 6 & -1.199148000 & 0.161393000 & -0.258547000 \\
\hline & 6 & -0.336935000 & 0.330295000 & -1.518702000 \\
\hline & 1 & -1.649483000 & 1.116030000 & 0.032305000 \\
\hline & 1 & -0.549422000 & -0.198895000 & 0.551700000 \\
\hline & 6 & -1.209176000 & 0.833555000 & -2.665997000 \\
\hline & 8 & -1.320467000 & 1.990742000 & -3.012837000 \\
\hline & 8 & -1.867373000 & -0.164322000 & -3.254733000 \\
\hline & 6 & 0.829921000 & 1.269039000 & -1.277010000 \\
\hline & 1 & 1.684591000 & 0.083242000 & -1.000222000 \\
\hline & 1 & 1.194719000 & 1.734970000 & -2.197872000 \\
\hline & 6 & -2.802512000 & 0.186659000 & -4.292416000 \\
\hline & 1 & -2.270548000 & 0.751169000 & -5.068128000 \\
\hline & 1 & -3.563122000 & 0.845329000 & -3.854064000 \\
\hline & 6 & -3.383036000 & -1.101849000 & -4.807243000 \\
\hline & 1 & -4.119738000 & -0.897246000 & -5.592233000 \\
\hline & 1 & -2.599371000 & -1.741934000 & -5.229855000 \\
\hline & 1 & -3.879084000 & -1.641789000 & -3.992369000 \\
\hline & 15 & 1.118656000 & 2.363339000 & 0.047196000 \\
\hline & 6 & 1.436848000 & 1.469516000 & 1.605420000 \\
\hline & 6 & 0.468680000 & 1.360789000 & 2.608686000 \\
\hline & 6 & 2.671921000 & 0.835277000 & 1.772355000 \\
\hline & 6 & 0.739808000 & 0.641189000 & 3.766618000 \\
\hline & 1 & -0.501376000 & 1.843090000 & 2.495390000 \\
\hline & 6 & 2.940257000 & 0.120589000 & 2.932970000 \\
\hline & 1 & 3.435295000 & 0.899689000 & 0.995813000 \\
\hline & 6 & 1.976985000 & 0.026177000 & 3.932422000 \\
\hline & 1 & -0.018475000 & 0.565000000 & 4.543224000 \\
\hline & 1 & 3.908022000 & -0.363230000 & 3.056005000 \\
\hline & 6 & -0.102790000 & 3.650584000 & 0.455726000 \\
\hline & 6 & -1.091838000 & 3.956296000 & -0.482729000 \\
\hline & 6 & -0.040230000 & 4.367160000 & 1.659287000 \\
\hline & 6 & -2.016064000 & 4.962260000 & -0.213232000 \\
\hline & 1 & -1.139688000 & 3.403267000 & -1.422099000 \\
\hline & 6 & -0.961218000 & 5.374104000 & 1.917162000 \\
\hline & 1 & 0.726601000 & 4.137933000 & 2.399291000 \\
\hline & 6 & -1.952196000 & 5.668745000 & 0.982540000 \\
\hline & 1 & -2.789867000 & 5.191228000 & -0.943091000 \\
\hline & 1 & -0.908897000 & 5.927562000 & 2.852380000 \\
\hline & 6 & 2.659709000 & 3.218718000 & -0.410317000 \\
\hline & 6 & 2.880245000 & 4.549059000 & -0.042138000 \\
\hline & 6 & 3.653082000 & 2.529451000 & -1.119225000 \\
\hline & 6 & 4.074734000 & 5.178728000 & -0.374922000 \\
\hline
\end{tabular}




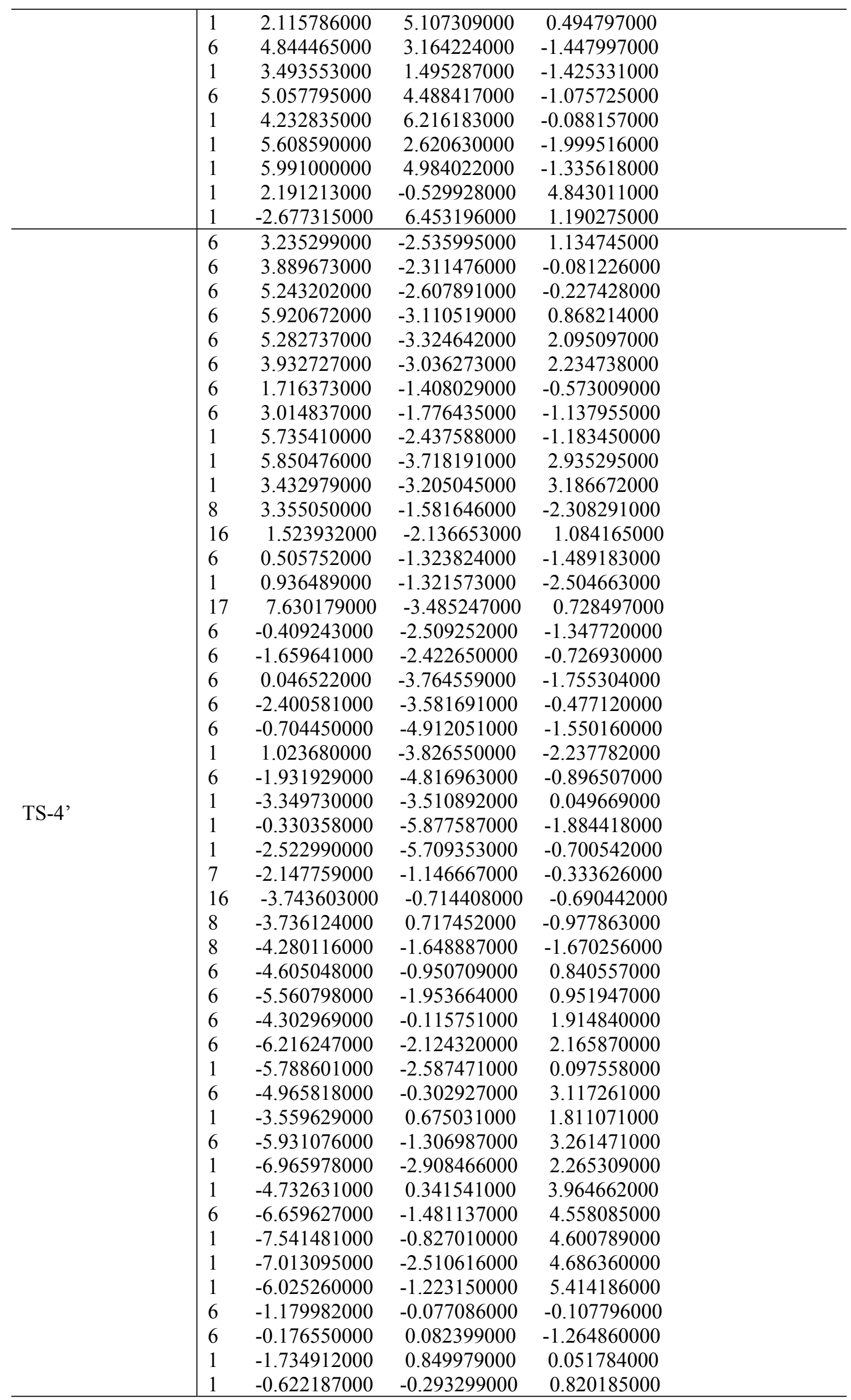




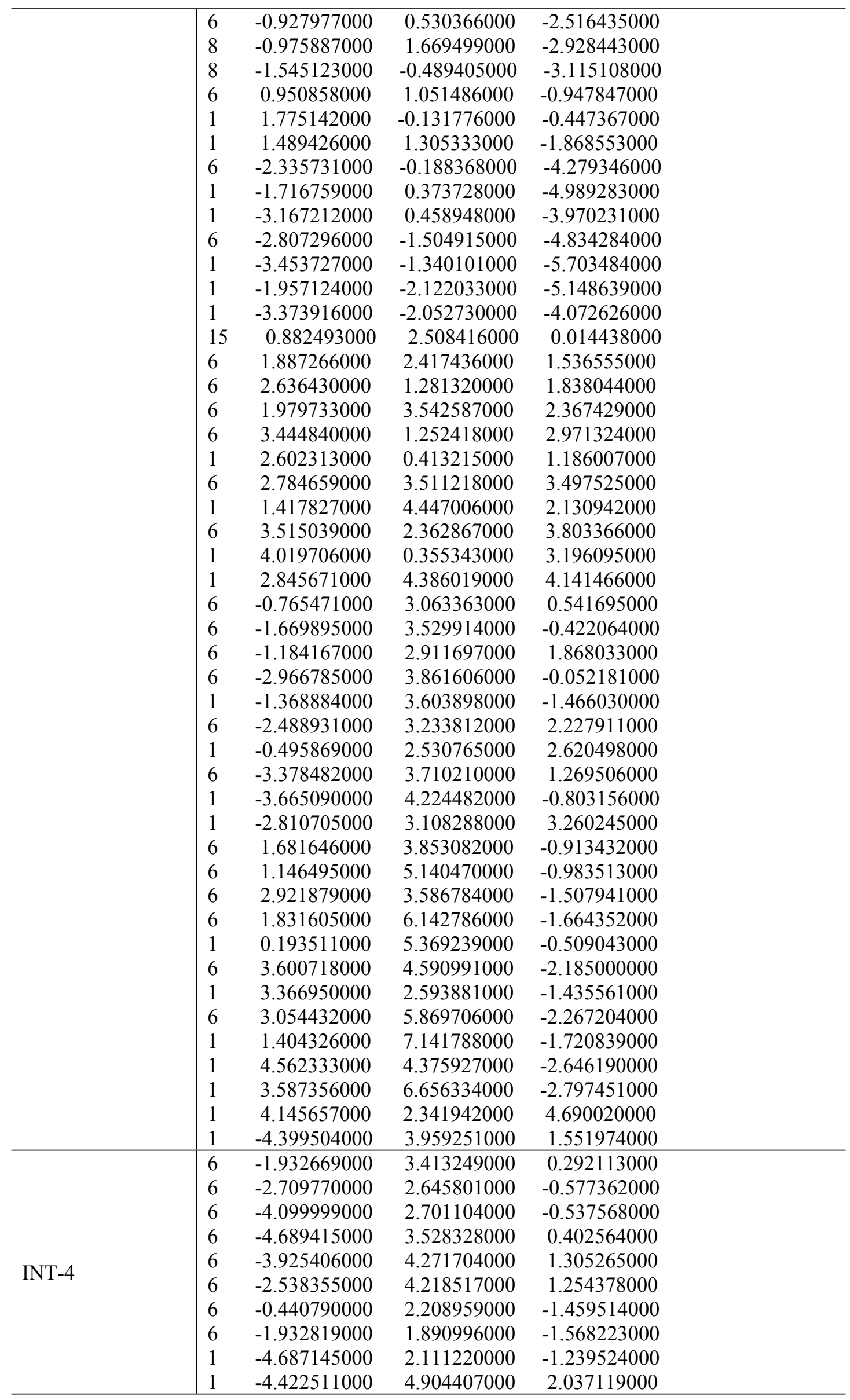




\begin{tabular}{|c|c|c|c|c|}
\hline & 1 & -1.943204000 & 4.814157000 & 1.943431000 \\
\hline & 8 & -2.400520000 & 1.269894000 & -2.511268000 \\
\hline & 16 & -0.192653000 & 3.275537000 & 0.028665000 \\
\hline & 6 & 0.469125000 & 0.986744000 & -1.623729000 \\
\hline & 1 & 0.032136000 & 0.500280000 & -2.509867000 \\
\hline & 17 & -6.435752000 & 3.671397000 & 0.459695000 \\
\hline & 6 & 1.885853000 & 1.383100000 & -1.987847000 \\
\hline & 6 & 3.004350000 & 1.045044000 & -1.207016000 \\
\hline & 6 & 2.100766000 & 2.086416000 & -3.177247000 \\
\hline & 6 & 4.282600000 & 1.442222000 & -1.620020000 \\
\hline & 6 & 3.366369000 & 2.464647000 & -3.595895000 \\
\hline & 1 & 1.239647000 & 2.322531000 & -3.804252000 \\
\hline & 6 & 4.463060000 & 2.137650000 & -2.804362000 \\
\hline & 1 & 5.150707000 & 1.209965000 & -1.009569000 \\
\hline & 1 & 3.496536000 & 3.003750000 & -4.531688000 \\
\hline & 1 & 5.467814000 & 2.429687000 & -3.103123000 \\
\hline & 7 & 2.817919000 & 0.326399000 & 0.007385000 \\
\hline & 16 & 3.922045000 & -0.850047000 & 0.497925000 \\
\hline & 8 & 3.299709000 & -1.559411000 & 1.611138000 \\
\hline & 8 & 4.386658000 & -1.601557000 & -0.664586000 \\
\hline & 6 & 5.284884000 & 0.067658000 & 1.163149000 \\
\hline & 6 & 6.581996000 & -0.256574000 & 0.784198000 \\
\hline & 6 & 5.030240000 & 1.050468000 & 2.117485000 \\
\hline & 6 & 7.641152000 & 0.427039000 & 1.370585000 \\
\hline & 1 & 6.753729000 & -1.025875000 & 0.034294000 \\
\hline & 6 & 6.098996000 & 1.721465000 & 2.688941000 \\
\hline & 1 & 4.005819000 & 1.289314000 & 2.401432000 \\
\hline & 6 & 7.417802000 & 1.422322000 & 2.323564000 \\
\hline & 1 & 8.662911000 & 0.183505000 & 1.081956000 \\
\hline & 1 & 5.914321000 & 2.494781000 & 3.433951000 \\
\hline & 6 & 8.558849000 & 2.175766000 & 2.933829000 \\
\hline & 1 & 8.417834000 & 2.309283000 & 4.013356000 \\
\hline & 1 & 9.513796000 & 1.663853000 & 2.771174000 \\
\hline & 1 & 8.643257000 & 3.179973000 & 2.496353000 \\
\hline & 6 & 1.469389000 & 0.318532000 & 0.582406000 \\
\hline & 6 & 0.395381000 & -0.042374000 & -0.464597000 \\
\hline & 1 & 1.445483000 & -0.403992000 & 1.398875000 \\
\hline & 1 & 1.258177000 & 1.313487000 & 0.994853000 \\
\hline & 6 & 0.787847000 & -1.441525000 & -0.947576000 \\
\hline & 8 & 0.849594000 & -2.399665000 & -0.205568000 \\
\hline & 8 & 1.101357000 & -1.505241000 & -2.238633000 \\
\hline & 6 & -0.971394000 & -0.040871000 & 0.179677000 \\
\hline & 1 & -0.244574000 & 2.870713000 & -2.316749000 \\
\hline & 1 & -1.116067000 & 0.739973000 & 0.932404000 \\
\hline & 6 & 1.568984000 & -2.780668000 & -2.720306000 \\
\hline & 1 & 0.808075000 & -3.541130000 & -2.499498000 \\
\hline & 1 & 2.481362000 & -3.037691000 & -2.165648000 \\
\hline & 6 & 1.811370000 & -2.630105000 & -4.197117000 \\
\hline & 1 & 2.190712000 & -3.570005000 & -4.613250000 \\
\hline & 1 & 0.881500000 & -2.372704000 & -4.718822000 \\
\hline & 1 & 2.549429000 & -1.843076000 & -4.392936000 \\
\hline & 15 & -1.999683000 & -1.364419000 & 0.487350000 \\
\hline & 6 & -3.649636000 & -0.664238000 & 0.810067000 \\
\hline & 6 & -3.850417000 & 0.066437000 & 1.987199000 \\
\hline & 6 & -4.710650000 & -0.843737000 & -0.077637000 \\
\hline & 6 & -5.098359000 & 0.607119000 & 2.269383000 \\
\hline & 1 & -3.031861000 & 0.200858000 & 2.695802000 \\
\hline
\end{tabular}




\begin{tabular}{|c|c|c|c|c|}
\hline & $\begin{array}{l}6 \\
1 \\
6 \\
1 \\
1 \\
6 \\
6 \\
6 \\
6 \\
1 \\
6 \\
1 \\
6 \\
1 \\
1 \\
6 \\
6 \\
6 \\
6 \\
1 \\
6 \\
1 \\
6 \\
1 \\
1 \\
1 \\
1 \\
1\end{array}$ & $\begin{array}{l}-5.962915000 \\
-4.565434000 \\
-6.158303000 \\
-5.247436000 \\
-6.788130000 \\
-1.778461000 \\
-0.534839000 \\
-2.864389000 \\
-0.376771000 \\
0.318497000 \\
-2.704791000 \\
-3.844379000 \\
-1.459908000 \\
0.597864000 \\
-3.554730000 \\
-2.114755000 \\
-2.083239000 \\
-2.193759000 \\
-2.133505000 \\
-1.998644000 \\
-2.245041000 \\
-2.219158000 \\
-2.212280000 \\
-2.102728000 \\
-2.309144000 \\
-2.246534000 \\
-7.137069000 \\
-1.336176000\end{array}$ & $\begin{array}{c}-0.306896000 \\
-1.413430000 \\
0.412982000 \\
1.179030000 \\
-0.459157000 \\
-2.454451000 \\
-2.550117000 \\
-3.165094000 \\
-3.342602000 \\
-2.019752000 \\
-3.960607000 \\
-3.095974000 \\
-4.047229000 \\
-3.411157000 \\
-4.510294000 \\
-2.491121000 \\
-3.879593000 \\
-1.940992000 \\
-4.709316000 \\
-4.316317000 \\
-2.776799000 \\
-0.857657000 \\
-4.159191000 \\
-5.789363000 \\
-2.343826000 \\
-4.809676000 \\
0.832166000 \\
-4.665161000\end{array}$ & $\begin{array}{c}0.212549000 \\
-0.994828000 \\
1.386037000 \\
3.183851000 \\
-0.480676000 \\
1.960283000 \\
2.586279000 \\
2.487071000 \\
3.719610000 \\
2.167017000 \\
3.615600000 \\
2.012727000 \\
4.235081000 \\
4.199594000 \\
4.015880000 \\
-0.936291000 \\
-0.780743000 \\
-2.221112000 \\
-1.895060000 \\
0.212671000 \\
-3.332991000 \\
-2.350129000 \\
-3.171963000 \\
-1.765816000 \\
-4.329710000 \\
-4.044034000 \\
1.611117000 \\
5.122582000\end{array}$ \\
\hline INT-4, & $\begin{array}{l}6 \\
6 \\
6 \\
6 \\
6 \\
6 \\
6 \\
6 \\
1 \\
1 \\
1 \\
8 \\
16 \\
6 \\
1 \\
17 \\
6 \\
6 \\
6 \\
6 \\
6 \\
1 \\
6 \\
1 \\
1 \\
1 \\
7 \\
16 \\
8\end{array}$ & $\begin{array}{c}-2.991527000 \\
-2.222472000 \\
-2.811904000 \\
-4.189985000 \\
-4.974789000 \\
-4.374124000 \\
-0.444276000 \\
-0.778626000 \\
-2.196568000 \\
-6.055004000 \\
-4.983658000 \\
0.086666000 \\
-2.049460000 \\
0.456058000 \\
0.087941000 \\
-4.975392000 \\
1.901955000 \\
2.987467000 \\
2.170924000 \\
4.290820000 \\
3.458247000 \\
1.329261000 \\
4.522628000 \\
5.137572000 \\
3.631108000 \\
5.545737000 \\
2.732882000 \\
3.882551000 \\
3.181331000 \\
\end{array}$ & $\begin{array}{c}-1.762978000 \\
-2.367428000 \\
-3.158327000 \\
-3.293994000 \\
-2.683877000 \\
-1.918192000 \\
-1.234838000 \\
-2.105680000 \\
-3.641624000 \\
-2.811089000 \\
-1.442526000 \\
-2.679421000 \\
-0.796693000 \\
0.013688000 \\
0.756165000 \\
-4.265083000 \\
-0.240808000 \\
-0.080095000 \\
-0.586776000 \\
-0.296160000 \\
-0.790974000 \\
-0.678421000 \\
-0.642487000 \\
-0.191134000 \\
-1.055212000 \\
-0.800095000 \\
0.269896000 \\
0.994016000 \\
1.436732000\end{array}$ & $\begin{array}{c}2.360281000 \\
1.366668000 \\
0.386251000 \\
0.405063000 \\
1.384294000 \\
2.377259000 \\
2.684568000 \\
1.472002000 \\
-0.370928000 \\
1.366690000 \\
3.143272000 \\
0.821947000 \\
3.498032000 \\
2.449763000 \\
3.173098000 \\
-0.827727000 \\
2.830735000 \\
1.950485000 \\
4.159081000 \\
2.421947000 \\
4.627126000 \\
4.848265000 \\
3.742518000 \\
1.751228000 \\
5.667971000 \\
4.078269000 \\
0.605859000 \\
-0.379677000 \\
-1.579860000\end{array}$ \\
\hline
\end{tabular}




\begin{tabular}{|c|c|c|c|c|}
\hline & 8 & 4.662453000 & 1.944952000 & 0.401479000 \\
\hline & 6 & 4.955490000 & -0.332933000 & -0.870741000 \\
\hline & 6 & 6.324850000 & -0.236312000 & -0.656246000 \\
\hline & 6 & 4.403762000 & -1.427687000 & -1.535015000 \\
\hline & 6 & 7.150684000 & -1.262068000 & -1.102782000 \\
\hline & 1 & 6.735197000 & 0.632232000 & -0.145142000 \\
\hline & 6 & 5.241934000 & -2.440561000 & -1.971477000 \\
\hline & 1 & 3.330300000 & -1.485502000 & -1.714518000 \\
\hline & 6 & 6.625088000 & -2.375387000 & -1.759750000 \\
\hline & 1 & 8.225711000 & -1.195120000 & -0.940219000 \\
\hline & 1 & 4.821117000 & -3.301623000 & -2.489803000 \\
\hline & 6 & 7.512699000 & -3.490641000 & -2.218512000 \\
\hline & 1 & 7.231322000 & -3.839477000 & -3.219621000 \\
\hline & 1 & 8.564613000 & -3.185003000 & -2.242318000 \\
\hline & 1 & 7.434575000 & -4.355226000 & -1.545272000 \\
\hline & 6 & 1.373427000 & 0.080655000 & 0.106743000 \\
\hline & 6 & 0.307986000 & 0.651415000 & 1.041260000 \\
\hline & 1 & 1.307963000 & 0.549243000 & -0.878849000 \\
\hline & 1 & 1.190719000 & -0.994727000 & -0.002355000 \\
\hline & 6 & 0.482800000 & 2.168087000 & 1.180333000 \\
\hline & 8 & 1.350402000 & 2.836679000 & 0.660512000 \\
\hline & 8 & -0.509439000 & 2.702858000 & 1.906069000 \\
\hline & 6 & -1.066416000 & 0.332425000 & 0.488504000 \\
\hline & 1 & 0.076989000 & -1.898653000 & 3.387962000 \\
\hline & 1 & -1.848725000 & 0.569136000 & 1.212118000 \\
\hline & 6 & -0.604160000 & 4.136868000 & 1.911541000 \\
\hline & 1 & -0.466437000 & 4.501575000 & 0.884274000 \\
\hline & 1 & 0.207473000 & 4.546441000 & 2.527133000 \\
\hline & 6 & -1.967038000 & 4.484284000 & 2.447228000 \\
\hline & 1 & -2.100386000 & 5.571880000 & 2.467805000 \\
\hline & 1 & -2.746770000 & 4.053529000 & 1.805057000 \\
\hline & 1 & -2.104109000 & 4.099987000 & 3.464878000 \\
\hline & 15 & -1.669528000 & 0.823392000 & -1.047160000 \\
\hline & 6 & -3.457199000 & 0.487997000 & -0.987750000 \\
\hline & 6 & -4.089817000 & -0.162735000 & -2.050147000 \\
\hline & 6 & -4.215804000 & 0.885222000 & 0.121191000 \\
\hline & 6 & -5.458242000 & -0.409997000 & -2.006730000 \\
\hline & 1 & -3.513051000 & -0.483227000 & -2.916584000 \\
\hline & 6 & -5.582442000 & 0.638843000 & 0.158523000 \\
\hline & 1 & -3.743391000 & 1.395124000 & 0.962324000 \\
\hline & 6 & -6.205396000 & -0.011035000 & -0.903686000 \\
\hline & 1 & -5.939521000 & -0.921356000 & -2.838106000 \\
\hline & 1 & -6.161425000 & 0.948829000 & 1.026419000 \\
\hline & 6 & -1.012893000 & -0.119454000 & -2.455126000 \\
\hline & 6 & -0.582770000 & -1.432792000 & -2.240254000 \\
\hline & 6 & -1.046349000 & 0.391430000 & -3.757169000 \\
\hline & 6 & -0.172314000 & -2.216526000 & -3.313277000 \\
\hline & 1 & -0.557982000 & -1.837180000 & -1.228492000 \\
\hline & 6 & -0.641098000 & -0.398386000 & -4.827314000 \\
\hline & 1 & -1.394104000 & 1.408042000 & -3.937706000 \\
\hline & 6 & -0.200273000 & -1.700513000 & -4.605672000 \\
\hline & 1 & 0.169470000 & -3.234792000 & -3.137862000 \\
\hline & 1 & -0.668501000 & 0.005581000 & -5.837192000 \\
\hline & 6 & -1.475517000 & 2.582771000 & -1.532314000 \\
\hline & 6 & -0.225721000 & 3.041226000 & -1.968266000 \\
\hline & 6 & -2.497357000 & 3.510454000 & -1.300023000 \\
\hline & 6 & -0.011356000 & 4.397500000 & -2.184371000 \\
\hline
\end{tabular}




\begin{tabular}{|c|c|c|c|c|}
\hline & $\begin{array}{l}1 \\
6 \\
1 \\
6 \\
1 \\
1 \\
1 \\
1 \\
1\end{array}$ & $\begin{array}{r}0.596393000 \\
-2.276834000 \\
-3.475825000 \\
-1.034900000 \\
0.962555000 \\
-3.081091000 \\
-0.864009000 \\
-7.275306000 \\
0.118429000 \\
\end{array}$ & $\begin{array}{r}2.341950000 \\
4.867947000 \\
3.174127000 \\
5.312764000 \\
4.741048000 \\
5.579345000 \\
6.374556000 \\
-0.207298000 \\
-2.315408000 \\
\end{array}$ & $\begin{array}{l}-2.131290000 \\
-1.508785000 \\
-0.958789000 \\
-1.953486000 \\
-2.527532000 \\
-1.330453000 \\
-2.120573000 \\
-0.869615000 \\
-5.445198000\end{array}$ \\
\hline $3 a$ & 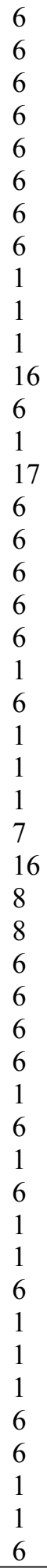 & $\begin{array}{c}4.160996000 \\
4.143769000 \\
5.154843000 \\
6.180834000 \\
6.210937000 \\
5.188543000 \\
2.388473000 \\
2.917687000 \\
5.149132000 \\
7.032643000 \\
5.205428000 \\
2.738048000 \\
0.943804000 \\
0.974107000 \\
7.479315000 \\
-0.094637000 \\
-1.265101000 \\
0.099126000 \\
-2.196666000 \\
-0.832506000 \\
1.000957000 \\
-1.987823000 \\
-3.083152000 \\
-0.658174000 \\
-2.721817000 \\
-1.464279000 \\
-2.914369000 \\
-2.590237000 \\
-3.556944000 \\
-3.915674000 \\
-5.107445000 \\
-3.493624000 \\
-5.884764000 \\
-5.418716000 \\
-4.281647000 \\
-2.561079000 \\
-5.486124000 \\
-6.821435000 \\
-3.964377000 \\
-6.315201000 \\
-7.479929000 \\
-5.811080000 \\
\end{array}$ & $\begin{array}{c}0.914917000 \\
0.225167000 \\
0.424173000 \\
1.305112000 \\
1.990491000 \\
1.798392000 \\
-0.927219000 \\
-0.555553000 \\
-0.100592000 \\
2.668050000 \\
2.334899000 \\
0.595152000 \\
-1.411745000 \\
-2.503180000 \\
1.553751000 \\
-0.894833000 \\
-0.251297000 \\
-1.086415000 \\
0.203469000 \\
-0.654764000 \\
-1.600927000 \\
-0.007641000 \\
0.737053000 \\
-0.820442000 \\
0.349524000 \\
-0.019546000 \\
-0.480223000 \\
-0.754583000 \\
-1.502807000 \\
0.981277000 \\
0.981689000 \\
2.105586000 \\
2.135199000 \\
0.091744000 \\
3.243765000 \\
2.087615000 \\
3.276983000 \\
2.147160000 \\
4.128039000 \\
4.523581000 \\
4.906387000 \\
\end{array}$ & $\begin{array}{c}1.069569000 \\
-0.158815000 \\
-1.089687000 \\
-0.765674000 \\
0.444394000 \\
1.370963000 \\
1.080557000 \\
-0.266930000 \\
-2.043077000 \\
0.663489000 \\
2.317459000 \\
2.098745000 \\
0.830487000 \\
0.956326000 \\
-1.918658000 \\
1.793123000 \\
1.373216000 \\
3.164608000 \\
2.310207000 \\
4.097102000 \\
3.500346000 \\
3.664056000 \\
1.972220000 \\
5.158034000 \\
4.383355000 \\
-0.016280000 \\
-0.747660000 \\
-2.145191000 \\
0.067406000 \\
-0.698604000 \\
0.015205000 \\
-1.408020000 \\
0.021126000 \\
0.557748000 \\
-1.389985000 \\
-1.970141000 \\
-0.674521000 \\
0.576911000 \\
-1.941906000 \\
-0.656256000 \\
-1.671351000 \\
-0.191022000 \\
-0.094639000 \\
-0.816166000 \\
-0.672752000 \\
-1.867120000 \\
-1.459046000 \\
\end{array}$ \\
\hline
\end{tabular}




\begin{tabular}{|c|c|c|c|c|}
\hline & $\begin{array}{l}8 \\
8 \\
6 \\
1 \\
1 \\
6 \\
1 \\
1 \\
6 \\
1 \\
1 \\
1 \\
\end{array}$ & $\begin{array}{r}0.222799000 \\
-0.760056000 \\
2.018349000 \\
2.993032000 \\
2.146702000 \\
-1.521212000 \\
-0.819493000 \\
-2.111278000 \\
-2.383267000 \\
-2.980197000 \\
-1.770166000 \\
-3.062142000\end{array}$ & $\begin{array}{l}-2.443580000 \\
-3.010282000 \\
-0.737635000 \\
-1.715530000 \\
-0.494964000 \\
-4.029433000 \\
-4.719562000 \\
-3.535760000 \\
-4.705889000 \\
-5.493680000 \\
-5.164917000 \\
-3.979769000 \\
\end{array}$ & $\begin{array}{r}-2.632252000 \\
-0.687907000 \\
-1.236178000 \\
1.549837000 \\
-2.289037000 \\
-1.371148000 \\
-1.855382000 \\
-2.153645000 \\
-0.341623000 \\
-0.814718000 \\
0.442860000 \\
0.120145000 \\
\end{array}$ \\
\hline $4 a$ & 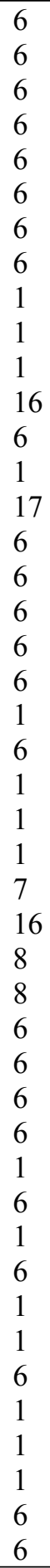 & $\begin{array}{c}4.667679000 \\
4.165633000 \\
5.010568000 \\
6.343884000 \\
6.850165000 \\
6.006319000 \\
2.103858000 \\
2.768230000 \\
4.635544000 \\
7.899555000 \\
6.397592000 \\
3.462728000 \\
0.981990000 \\
1.414187000 \\
7.419743000 \\
-0.183619000 \\
-1.440357000 \\
0.000770000 \\
-2.471401000 \\
-1.026680000 \\
0.976935000 \\
-2.269183000 \\
-3.436040000 \\
-0.859331000 \\
-3.082654000 \\
-1.636561000 \\
-3.010702000 \\
-2.625966000 \\
-3.554445000 \\
-4.170672000 \\
-5.386022000 \\
-3.853066000 \\
-6.294358000 \\
-5.614687000 \\
-4.770647000 \\
-2.900533000 \\
-6.002003000 \\
-7.250807000 \\
-4.534072000 \\
-6.972182000 \\
-7.953034000 \\
-6.609183000 \\
-7.66426000 \\
\end{array}$ & $\begin{array}{c}-0.084441000 \\
0.663814000 \\
1.499974000 \\
1.577872000 \\
0.843704000 \\
0.001430000 \\
-0.297102000 \\
0.359564000 \\
2.083683000 \\
0.932673000 \\
-0.575737000 \\
-1.143478000 \\
-1.133856000 \\
-2.100436000 \\
2.637571000 \\
-1.372542000 \\
-0.787384000 \\
-2.172810000 \\
-0.999849000 \\
-2.403725000 \\
-2.635714000 \\
-1.811712000 \\
-0.519615000 \\
-3.037553000 \\
-1.970405000 \\
0.052125000 \\
-0.132406000 \\
0.311835000 \\
-1.469571000 \\
1.038323000 \\
0.595834000 \\
2.395668000 \\
1.533133000 \\
-0.467626000 \\
3.313014000 \\
2.731627000 \\
2.897023000 \\
3.3766705000 \\
3.457020000 \\
4.336709000 \\
\end{array}$ & $\begin{array}{c}1.197250000 \\
0.116157000 \\
-0.607106000 \\
-0.228054000 \\
0.840969000 \\
1.559551000 \\
1.043991000 \\
-0.130372000 \\
-1.445497000 \\
1.111536000 \\
2.394597000 \\
1.970599000 \\
0.408394000 \\
0.104594000 \\
-1.120132000 \\
1.328800000 \\
1.122366000 \\
2.458439000 \\
2.041559000 \\
3.361239000 \\
2.615945000 \\
3.146928000 \\
1.889743000 \\
4.229260000 \\
3.851827000 \\
-0.008287000 \\
-0.970411000 \\
-2.307111000 \\
-0.775091000 \\
-0.316806000 \\
0.190458000 \\
-0.358460000 \\
0.671136000 \\
0.206470000 \\
0.125367000 \\
-0.765909000 \\
0.648895000 \\
1.069267000 \\
0.098866000 \\
1.182987000 \\
1.376912000 \\
-0.8615342000 \\
0.4830000 \\
\end{array}$ \\
\hline
\end{tabular}




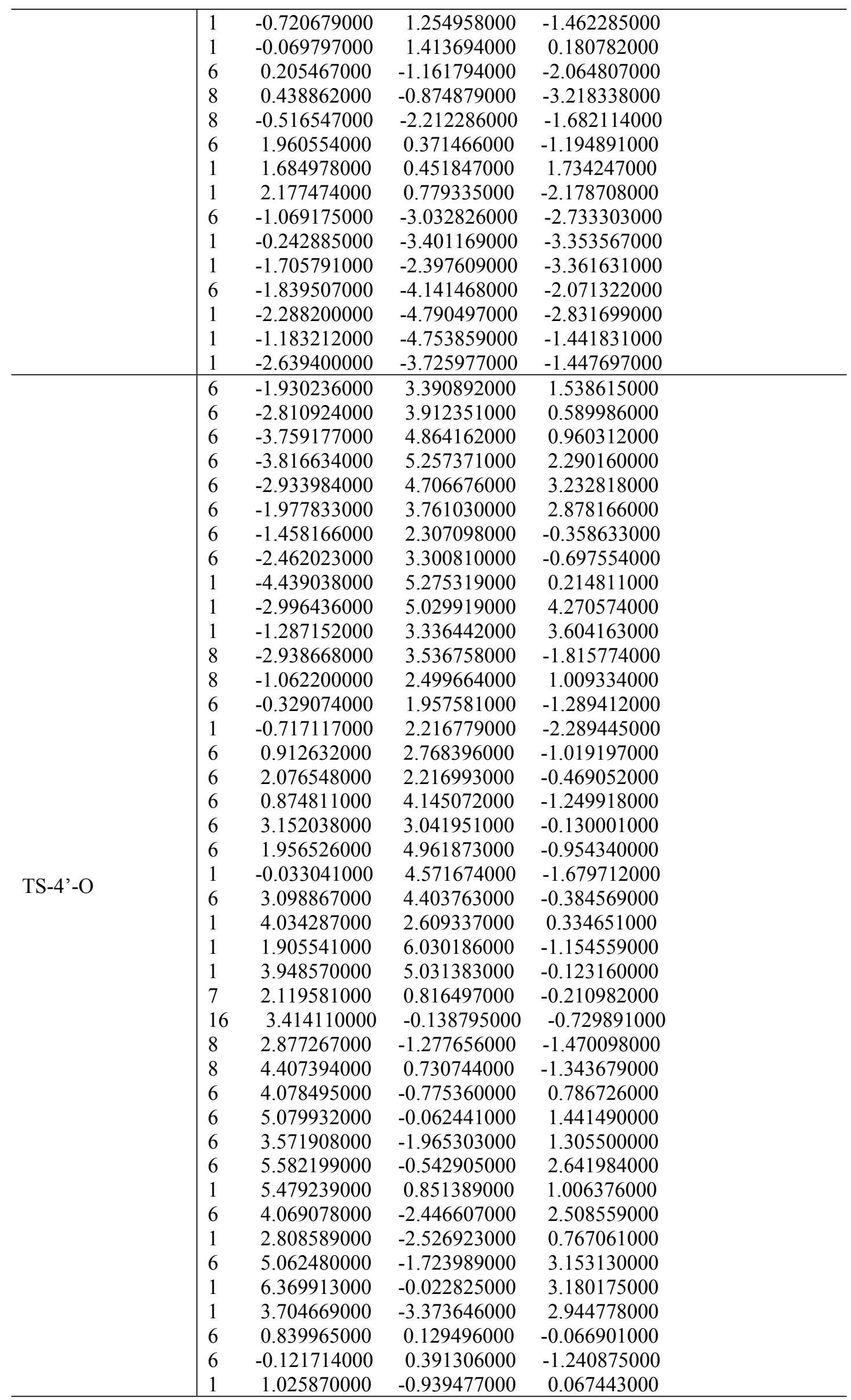




\begin{tabular}{|c|c|c|c|c|}
\hline & 1 & 0.365539000 & 0.498306000 & 0.854158000 \\
\hline & 6 & 0.457897000 & -0.098124000 & -2.566738000 \\
\hline & 8 & 0.101985000 & -1.091962000 & -3.164749000 \\
\hline & 8 & 1.396323000 & 0.730422000 & -3.022001000 \\
\hline & 6 & -1.500373000 & -0.202751000 & -1.009862000 \\
\hline & 1 & -1.965672000 & 1.046488000 & -0.438470000 \\
\hline & 1 & -2.052548000 & -0.238371000 & -1.955728000 \\
\hline & 6 & 2.050030000 & 0.374956000 & -4.252911000 \\
\hline & 1 & 1.285387000 & 0.219976000 & -5.024150000 \\
\hline & 1 & 2.575105000 & -0.576241000 & -4.096602000 \\
\hline & 6 & 2.986143000 & 1.504881000 & -4.586752000 \\
\hline & 1 & 3.516718000 & 1.293831000 & -5.521969000 \\
\hline & 1 & 2.433367000 & 2.444361000 & -4.707448000 \\
\hline & 1 & 3.726141000 & 1.638763000 & -3.788757000 \\
\hline & 15 & -1.909648000 & -1.688980000 & -0.166945000 \\
\hline & 6 & -2.740827000 & -1.381436000 & 1.422991000 \\
\hline & 6 & -2.494060000 & -0.214914000 & 2.149466000 \\
\hline & 6 & -3.617632000 & -2.345828000 & 1.934391000 \\
\hline & 6 & -3.117793000 & -0.018171000 & 3.378175000 \\
\hline & 1 & -1.820671000 & 0.549848000 & 1.762483000 \\
\hline & 6 & -4.236831000 & -2.144323000 & 3.161192000 \\
\hline & 1 & -3.816964000 & -3.259295000 & 1.373266000 \\
\hline & 6 & -3.987208000 & -0.979139000 & 3.883123000 \\
\hline & 1 & -2.925022000 & 0.895921000 & 3.936897000 \\
\hline & 1 & -4.920089000 & -2.895094000 & 3.552474000 \\
\hline & 6 & -0.577877000 & -2.878628000 & 0.195118000 \\
\hline & 6 & 0.256732000 & -3.301584000 & -0.849246000 \\
\hline & 6 & -0.388476000 & -3.387181000 & 1.483776000 \\
\hline & 6 & 1.248325000 & -4.243832000 & -0.604704000 \\
\hline & 1 & 0.142736000 & -2.874228000 & -1.845914000 \\
\hline & 6 & 0.609388000 & -4.328155000 & 1.721053000 \\
\hline & 1 & -1.020057000 & -3.054581000 & 2.305430000 \\
\hline & 6 & 1.419906000 & -4.763887000 & 0.677385000 \\
\hline & 1 & 1.893233000 & -4.569292000 & -1.418346000 \\
\hline & 1 & 0.750481000 & -4.722542000 & 2.725253000 \\
\hline & 6 & -3.144439000 & -2.551340000 & -1.178704000 \\
\hline & 6 & -2.993813000 & -3.887057000 & -1.553659000 \\
\hline & 6 & -4.287421000 & -1.842786000 & -1.570497000 \\
\hline & 6 & -3.969212000 & -4.503034000 & -2.332746000 \\
\hline & 1 & -2.116773000 & -4.451064000 & -1.240552000 \\
\hline & 6 & -5.254808000 & -2.462487000 & -2.350143000 \\
\hline & 1 & -4.426608000 & -0.806430000 & -1.261433000 \\
\hline & 6 & -5.094758000 & -3.792475000 & -2.733968000 \\
\hline & 1 & -3.845930000 & -5.543782000 & -2.624499000 \\
\hline & 1 & -6.139296000 & -1.906831000 & -2.654243000 \\
\hline & 1 & -5.855298000 & -4.276837000 & -3.342964000 \\
\hline & 1 & -4.476036000 & -0.819989000 & 4.842335000 \\
\hline & 1 & 2.198142000 & -5.501738000 & 0.864516000 \\
\hline & 9 & 5.543749000 & -2.186212000 & 4.306830000 \\
\hline & 1 & -4.550076000 & 5.993840000 & 2.612269000 \\
\hline & 6 & -3.198276000 & 3.433402000 & 0.008807000 \\
\hline & 6 & -2.608206000 & 3.174217000 & 1.248164000 \\
\hline & 6 & -3.132864000 & 3.739103000 & 2.409686000 \\
\hline TS-4-O & 6 & -4.258072000 & 4.544372000 & 2.304761000 \\
\hline & 6 & -4.844955000 & 4.783504000 & 1.050855000 \\
\hline & 6 & -4.331141000 & 4.232912000 & -0.117136000 \\
\hline & 6 & -1.534649000 & 2.009016000 & -0.414744000 \\
\hline
\end{tabular}




\begin{tabular}{|c|c|c|c|c|}
\hline & 6 & -1.441450000 & 2.323254000 & 0.996223000 \\
\hline & 1 & -2.663773000 & 3.534655000 & 3.372425000 \\
\hline & 1 & -5.727569000 & 5.418559000 & 0.991736000 \\
\hline & 1 & -4.784453000 & 4.417613000 & -1.088744000 \\
\hline & 8 & -0.599622000 & 1.921628000 & 1.816435000 \\
\hline & 8 & -2.557546000 & 2.824053000 & -1.009492000 \\
\hline & 6 & -0.356201000 & 1.750719000 & -1.321095000 \\
\hline & 1 & -0.684666000 & 2.067583000 & -2.323306000 \\
\hline & 6 & 0.870847000 & 2.550583000 & -0.952651000 \\
\hline & 6 & 2.048135000 & 1.972788000 & -0.462826000 \\
\hline & 6 & 0.813659000 & 3.941664000 & -1.059681000 \\
\hline & 6 & 3.122880000 & 2.778598000 & -0.079475000 \\
\hline & 6 & 1.889299000 & 4.745855000 & -0.711511000 \\
\hline & 1 & -0.108462000 & 4.392219000 & -1.430964000 \\
\hline & 6 & 3.050805000 & 4.156588000 & -0.217311000 \\
\hline & 1 & 4.018836000 & 2.323371000 & 0.337065000 \\
\hline & 1 & 1.819929000 & 5.826803000 & -0.815519000 \\
\hline & 1 & 3.899981000 & 4.770401000 & 0.076606000 \\
\hline & 7 & 2.115677000 & 0.556755000 & -0.333349000 \\
\hline & 16 & 3.475228000 & -0.286685000 & -0.851119000 \\
\hline & 8 & 3.006546000 & -1.609302000 & -1.258058000 \\
\hline & 8 & 4.238590000 & 0.532136000 & -1.782839000 \\
\hline & 6 & 4.454356000 & -0.493258000 & 0.615214000 \\
\hline & 6 & 5.718660000 & 0.083301000 & 0.685718000 \\
\hline & 6 & 3.941945000 & -1.259755000 & 1.661775000 \\
\hline & 6 & 6.487139000 & -0.101027000 & 1.828187000 \\
\hline & 1 & 6.095628000 & 0.669870000 & -0.148885000 \\
\hline & 6 & 4.705242000 & -1.445925000 & 2.802855000 \\
\hline & 1 & 2.954275000 & -1.712120000 & 1.586626000 \\
\hline & 6 & 5.963842000 & -0.860660000 & 2.862259000 \\
\hline & 1 & 7.479096000 & 0.331690000 & 1.924997000 \\
\hline & 1 & 4.345953000 & -2.034718000 & 3.642291000 \\
\hline & 6 & 0.843520000 & -0.155436000 & -0.229225000 \\
\hline & 6 & -0.118647000 & 0.183739000 & -1.380234000 \\
\hline & 1 & 1.047160000 & -1.230254000 & -0.195443000 \\
\hline & 1 & 0.373285000 & 0.164858000 & 0.713616000 \\
\hline & 6 & 0.504254000 & -0.221816000 & -2.713078000 \\
\hline & 8 & 0.286066000 & -1.258517000 & -3.305125000 \\
\hline & 8 & 1.346496000 & 0.706686000 & -3.162642000 \\
\hline & 6 & -1.476712000 & -0.474307000 & -1.200333000 \\
\hline & 1 & -1.955671000 & 0.691532000 & -0.579254000 \\
\hline & 1 & -1.994629000 & -0.622146000 & -2.152874000 \\
\hline & 6 & 2.101891000 & 0.397391000 & -4.349613000 \\
\hline & 1 & 1.402177000 & 0.149292000 & -5.156909000 \\
\hline & 1 & 2.711914000 & -0.490857000 & -4.139045000 \\
\hline & 6 & 2.941514000 & 1.606799000 & -4.660275000 \\
\hline & 1 & 3.547897000 & 1.421652000 & -5.554135000 \\
\hline & 1 & 2.309694000 & 2.482724000 & -4.850206000 \\
\hline & 1 & 3.612388000 & 1.829062000 & -3.821368000 \\
\hline & 15 & -1.961331000 & -1.760344000 & -0.113515000 \\
\hline & 6 & -2.010297000 & -1.176686000 & 1.610345000 \\
\hline & 6 & -0.955569000 & -1.423281000 & 2.494792000 \\
\hline & 6 & -3.086759000 & -0.386834000 & 2.025639000 \\
\hline & 6 & -0.979740000 & -0.883661000 & 3.774721000 \\
\hline & 1 & -0.112251000 & -2.043040000 & 2.191054000 \\
\hline & 6 & -3.106165000 & 0.150887000 & 3.306381000 \\
\hline & 1 & -3.920097000 & -0.190695000 & 1.351639000 \\
\hline
\end{tabular}




\begin{tabular}{r|rrrr}
\hline 6 & -2.052350000 & -0.095381000 & 4.179883000 \\
1 & -0.157547000 & -1.083268000 & 4.458834000 \\
1 & -3.952599000 & 0.757101000 & 3.622796000 \\
6 & -1.034756000 & -3.322067000 & -0.084786000 \\
6 & -0.141484000 & -3.605220000 & -1.120681000 \\
6 & -1.232920000 & -4.253498000 & 0.943888000 \\
6 & 0.554482000 & -4.811105000 & -1.120896000 \\
1 & 0.014409000 & -2.877781000 & -1.919332000 \\
6 & -0.541364000 & -5.457426000 & 0.930746000 \\
1 & -1.924519000 & -4.036325000 & 1.758219000 \\
6 & 0.355228000 & -5.734007000 & -0.100003000 \\
1 & 1.257405000 & -5.026555000 & -1.922974000 \\
1 & -0.697089000 & -6.180130000 & 1.728802000 \\
6 & -3.666233000 & -2.137678000 & -0.618469000 \\
6 & -4.151793000 & -3.447461000 & -0.583898000 \\
6 & -4.515459000 & -1.098428000 & -1.024379000 \\
6 & -5.467869000 & -3.714468000 & -0.945938000 \\
1 & -3.503631000 & -4.269491000 & -0.286117000 \\
6 & -5.829522000 & -1.372340000 & -1.382313000 \\
1 & -4.149776000 & -0.072725000 & -1.068905000 \\
6 & -6.307442000 & -2.679294000 & -1.342709000 \\
1 & -5.834380000 & -4.738364000 & -0.920763000 \\
1 & -6.481127000 & -0.559381000 & -1.695111000 \\
1 & -7.336958000 & -2.891035000 & -1.624213000 \\
1 & -2.069356000 & 0.323821000 & 5.184125000 \\
1 & 0.902896000 & -6.674375000 & -0.103122000 \\
9 & 6.698098000 & -1.039683000 & 3.960416000 \\
1 & -4.695728000 & 4.992934000 & 3.194644000 \\
\hline & & & \\
& & & & \\
& &
\end{tabular}

Table S2. Electronic energies, entropies and Gibbs free-energies (at $353.15 \mathrm{~K}$ in $\mathrm{CH}_{3} \mathrm{CN}$ solvent) computed at the m06-PCM/6-31G* level of theory.

\begin{tabular}{llll}
\hline Species Name & $\boldsymbol{E}$ in a.u. & $\boldsymbol{S}$ in $\mathbf{~ c a l} / \mathbf{m o l} \cdot \mathbf{K}$ & $\boldsymbol{G}$ in a.u. \\
\hline $\mathbf{1 a}$ & -2384.07422 & 182.630 & -2383.81089 \\
$\mathbf{2}$ & -882.06522 & 160.131 & -881.81636 \\
PR $_{3}$ & -1035.72295 & 134.277 & -1035.50257 \\
$\mathbf{G}$ & -1419.34778 & 179.431 & -1419.00871 \\
$\mathbf{C O}_{2}$ & -188.50463 & 52.625 & -188.51799 \\
'BuOH & -233.50931 & 81.473 & -233.40949 \\
TS-1 & -3803.44938 & 290.824 & -3802.80965 \\
INT-1 & -3803.47469 & 298.081 & -3802.83303 \\
TS-2 & -3803.42762 & 297.847 & -3802.78698 \\
INT-2 & -2767.74011 & 222.966 & -2767.35398 \\
TS-3 & -3803.47049 & 288.334 & -3802.8229 \\
INT-3 & -3803.49086 & 288.586 & -3802.84113 \\
TS-4' & -3803.45534 & 290.178 & -3802.81252 \\
TS-4 & -3803.45806 & 290.184 & -3802.81483 \\
INT-4 & -3803.47347 & 285.730 & -3802.82362 \\
INT-4 & -3803.47872 & 289.314 & -3802.83022 \\
4a & -2692.57067 & 206.165 & -2692.17894 \\
3a & -2692.56376 & 206.589 & -2692.17227 \\
PR & -1110.95724 & 138.601 & -1110.73289 \\
TS-4'-O & -3080.83348 & 276.510 & -3080.20885 \\
TS-4-O & -3080.83221 & 274.488 & -3080.20675 \\
\hline
\end{tabular}


Table S3. Imaginary frequencies $\left(\mathrm{cm}^{-1}\right)$ of the transition states, computed at the m06$\mathrm{PCM} / 6-31 \mathrm{G}^{*}$ level of theory. All of the minimal structures do not have any imaginary frequencies.

\begin{tabular}{cccc}
\hline Species Name & $\boldsymbol{I}$. $\boldsymbol{F}$. & Species Name & I. $\boldsymbol{F}$. \\
\hline TS-1 & $-735.9 i$ & TS-2 & $-514.5 i$ \\
TS-3 & $-269.3 i$ & TS-4 & $-1158.8 i$ \\
TS-4 & $-1275.7 i$ & TS-4'-O & $-1180.3 i$ \\
TS-4-O & $-1016.0 i$ & & \\
\hline
\end{tabular}
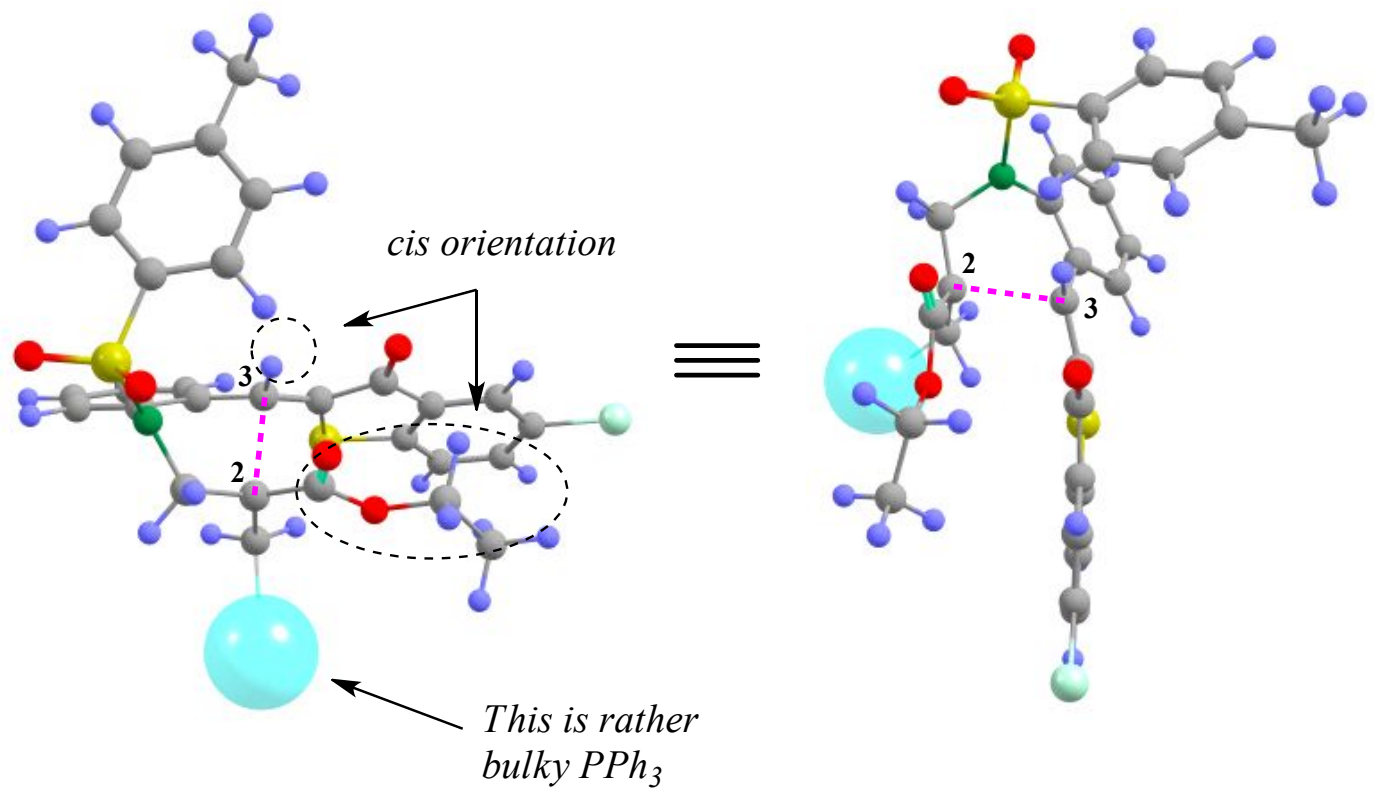

Figure S1. Three-dimensional structure of the only located TS-3 transition state

\section{Computational Methods}

All calculations were finished using the Gaussian 09 computational program ${ }^{[1]}$. Geometrical optimizations were performed by the $\mathrm{m} 06$ density functional method ${ }^{[2]}$ with the 6-31g* basis set for all elements. The default self-consistent reaction field polarizable continuum model ${ }^{[3]}$ was used to consider the implicit solvation effects of $\mathrm{CH}_{3} \mathrm{CN}$. All of the resultant stationary point geometries were characterized by vibrational analyses, from which zero point energies and Gibbs free-energies were obtained, in addition to confirming whether all of the structures resided at minima or first-order saddle points on the potential energy surfaces. The contributions of smallfrequency vibrations to the computed entropies were corrected by the quasi-RRHO approach. ${ }^{[4]}$ Considering the default entropic data obtained from the Gaussian output files are the idea-gas-phase entropies, which would exaggerate the activation entropies for the bimolecular reaction in solution. ${ }^{[5]}$ Hence, the default entropies are scaled by a factor of 0.5 in Gibbs free-energy determinations. 


\section{References}

(1) Frisch, M. J.; Trucks, G. W.; Schlegel, H. B.; Scuseria, G. E.; Robb, M. A.; Cheeseman, J. R.; Scalmani, G.; Barone, V.; Mennucci, B.; Petersson, G. A.; Nakatsuji, H.; Caricato, M.; Li, X.; Hratchian, H. P.; Izmaylov, A. F.; Bloino, J.; Zheng, G.; Sonnenberg, J. L.; Hada, M.; Ehara, M.; Toyota, K.; Fukuda, R.; Hasegawa, J.; Ishida, M.; Nakajima, T.; Honda, Y.; Kitao, O.; Nakai, H.; Vreven, T.; Montgomery, J. A., Jr.; Peralta, J. E.; Ogliaro, F.; Bearpark, M.; Heyd, J. J.; Brothers, E.; Kudin, K. N.; Staroverov, V. N.; Kobayashi, R.; Normand, J.; Raghavachari, K.; Rendell, A.; Burant, J. C.; Iyengar, S. S.; Tomasi, J.; Cossi, M.; Rega, N.; Millam, J. M.; Klene, M.; Knox, J. E.; Cross, J. B.; Bakken, V.; Adamo, C.; Jaramillo, J.; Gomperts, R.; Stratmann, R. E.; Yazyev, O.; Austin, A. J.; Cammi, R.; Pomelli, C.; Ochterski, J. W.; Martin, R. L.; Morokuma, K.; Zakrzewski, V. G.; Voth, G. A.; Salvador, P.; Dannenberg, J. J.; Dapprich, S.; Daniels, A. D.; Farkas, O.; Foresman, J. B.; Ortiz, J. V.; Cioslowski, J.; Fox, D. J. Gaussian 09, Revision A.02, Gaussian, Inc., Wallingford, CT, 2009.

(2) Zhao, Y.; Truhlar, D. G. A New Local Density Functional for Main-Group Thermochemistry, Transition Metal Bonding, Thermochemical Kinetics, and Noncovalent Interactions. J. Chem. Phys. 2006, 125, 194101.

(3) Scalmani, G.; Frisch, M. J. Continuous Surface Charge Polarizable Continuum Models of Solvation. I. General Formalism. J. Chem. Phys. 2010, 132, 114110.

(4) Grimme, S. Supramolecular Binding Thermodynamics by Dispersion-Corrected Density Functional Theory. Chem. Eur. J. 2012, 18, 9955.

(5) (a) Huang, F.; Lu, G.; Zhao, L.; Li, H.; Wang, Z.-X. The Catalytic Role of NHeterocyclic Carbene in a Metal-Free Conversion of Carbon Dioxide into Methanol: A Computational Mechanism Study. J. Am. Chem. Soc. 2010, 132, 12388; (b) Plata, R. E.; Singleton, D. A. A Case Study of the Mechanism of Alcohol-Mediated Morita Baylis-Hillman Reactions. The Importance of Experimental Observations. J. Am. Chem. Soc. 2015, 137, 3811; (c) J. Kua, J.; Rodriguez, A. A.; Marucci, L. A.; Galloway, M. M.; De Haan, D. O. Free Energy Map for the Co-Oligomerization of Formaldehyde and Ammonia. J. Phys. Chem. A 2015, 119, 2122.

\section{X-ray Data Collection and Structure Determinations}

Single crystals of $\mathbf{3 a}, \mathbf{4 a}$, and $\mathbf{7 f}$ were grown by slow diffusion of $n$-hexane into a dichloromethane solution. X-ray single-crystal diffraction data was collected on a Rigaku XtaLAB P200 diffractometer at 293(2) K with MoK $\alpha$ radiation $(\lambda=0.71073 \AA)$ in the $\omega$ scan mode. The program SAINT was used for integration of the diffraction profiles. All of the structures were solved using direct methods using the SHELXS program of the SHELXTL package and refined using full matrix least-squares methods with SHELXL (semi empirical absorption corrections were applied using the 
SADABS program). Other non-hydrogen atoms were located in successive difference Fourier syntheses and refined with anisotropic thermal parameters on F2. The hydrogen atoms were generated theoretically onto the specific atoms and refined isotopically with fixed thermal factors. Detailed crystallographic data were summarized in Table S4.

Table S4 Crystal Date and Structure Refinements for 3a, 4a and 7f

\begin{tabular}{|c|c|c|c|}
\hline & $3 \mathbf{a}$ & $4 \mathbf{a}$ & $7 f$ \\
\hline Chemical formula & $\mathrm{C}_{28} \mathrm{H}_{24} \mathrm{ClNO}_{4} \mathrm{~S}_{2}$ & $\mathrm{C}_{28} \mathrm{H}_{24} \mathrm{ClNO}_{4} \mathrm{~S}_{2}$ & $\mathrm{C}_{27} \mathrm{H}_{21} \mathrm{ClFNO}_{5} \mathrm{~S}$ \\
\hline Formula weight & 538.05 & 538.05 & 525.96 \\
\hline Crystal system & monoclinic & triclinic & triclinic \\
\hline Space group & P $121 / \mathrm{n} 1$ & $P-1$ & $\mathrm{P}-1$ \\
\hline$a(\AA)$ & $10.9679(17)$ & $8.0583(8)$ & $8.0056(14)$ \\
\hline$b(\AA)$ & $17.281(3)$ & $10.8415(10)$ & $11.2427(19)$ \\
\hline$c(\AA)$ & $14.158(3)$ & $14.8495(14)$ & $13.774(3)$ \\
\hline$V\left(\AA^{3}\right)$ & $2573.6(8)$ & $1281.5(2)$ & $1220.9(4)$ \\
\hline$\alpha\left(\left(^{\circ}\right)\right.$ & 90 & $91.482(8)$ & $88.955(15)$ \\
\hline$\beta\left(\left(^{\circ}\right)\right.$ & $106.458(17)$ & $91.077(8)$ & $80.052(16)$ \\
\hline$\gamma\left(\left(^{\circ}\right)\right.$ & 90 & $98.721(8)$ & $89.787(14)$ \\
\hline$Z$ & 4 & 2 & 2 \\
\hline $\mathrm{F}(000)$ & 1120.0 & 560.0 & 544.0 \\
\hline GOF & 0.870 & 1.047 & 1.015 \\
\hline$D / \mathrm{g} \mathrm{cm}^{-3}$ & 1.389 & 1.394 & 1.431 \\
\hline$\mu\left(\mathrm{mm}^{-1}\right)$ & 0.346 & 0.348 & 0.290 \\
\hline$T / K$ & 293 & 293 & 293 \\
\hline$\Lambda M o K \mid a(\AA)$ & 0.71073 & 0.71073 & 0.71073 \\
\hline$R^{a} / R^{b}$ & $0.0705 / 0.2326$ & $0.0393 / 0.1052$ & $0.0906 / 0.2752$ \\
\hline \multicolumn{4}{|c|}{${ }^{a} R=\Sigma|| F_{\mathrm{o}}|-| F_{\mathrm{c}}|| / \Sigma\left|F_{\mathrm{o}}\right| \cdot{ }^{b} R_{w}=\left[\Sigma\left[w\left(F_{o}{ }^{2}-F_{c}{ }^{2}\right)^{2}\right] / \Sigma w\left(\mathrm{Fo}^{2}\right)^{2}\right]^{1 / 2}$} \\
\hline
\end{tabular}


5. X-ray Crystal Structures (30\% thermal ellipsoid probability levels)

3a

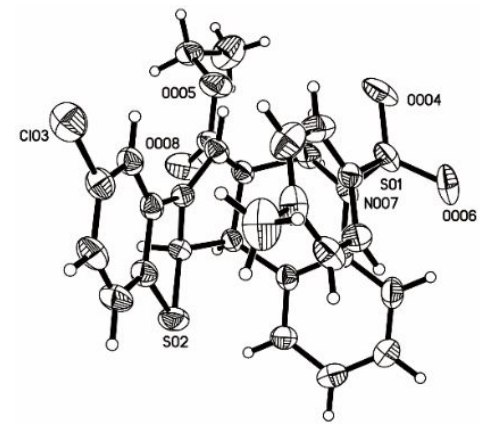<smiles>CCOC1(C)C=C2c3cc(Cl)ccc3S[C@H]2C12CN([As])c1ccccc12</smiles>

$3 a$

$4 a$

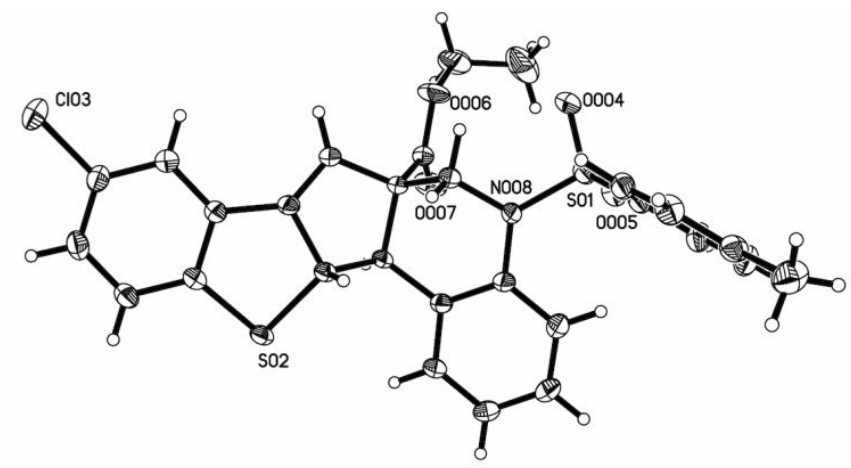

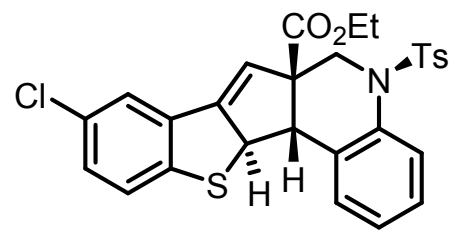

4a

$7 \mathbf{f}$
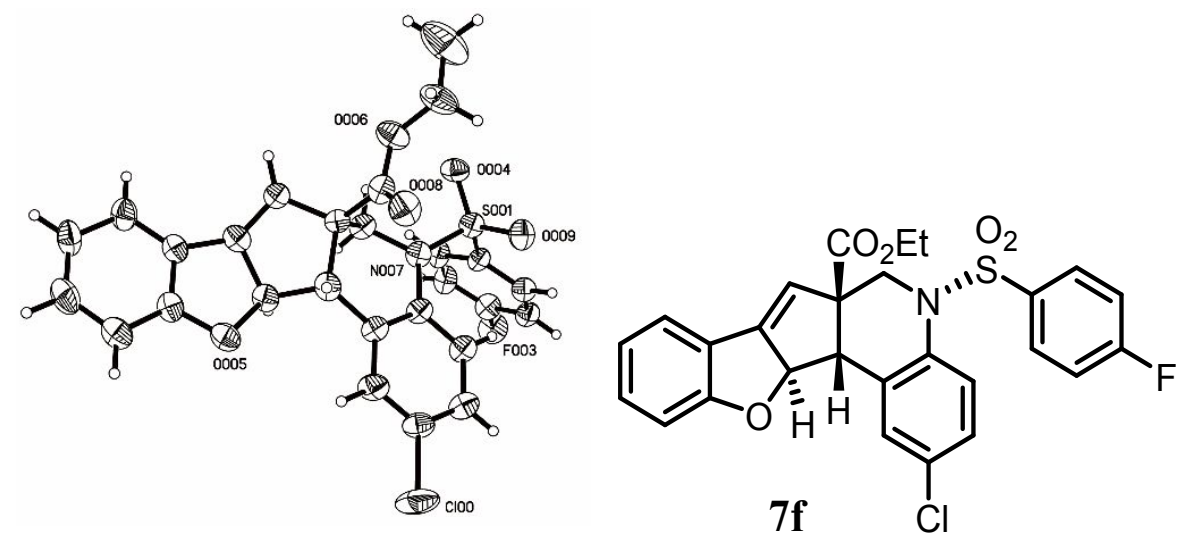

6. Some equations in chemical kinetics to determine the difference of the freeenergy barriers of two competing reactions from the product ratio observed

$$
k=\frac{k_{B} T}{h}\left(c^{\theta}\right)^{1-n} \exp \left(-\frac{\Delta G^{\ddagger}}{R T}\right)
$$

This is the central equation of the transition state theory, in which $k$ is the rate constant, $k_{B}$ is the Boltzmann constant, $h$ is the Planck constant, $c^{\theta}$ is the standard 
concentration and $\Delta G^{t}$ is the free-energy barrier. Using this equation, we can derive the rate constant from a given free-energy barrier or do a reserve process.

Under kinetics-controlled conditions, the product ratio of two competing reactions is equal to $k_{1}: k_{2}$, which is also equal to the following on the basis of the above equation. Then, we can derive the difference of two barriers from the product ratio or do a reverse process.

product ratio $=\frac{k_{1}}{k_{2}}=\exp \left[\left(\Delta G_{2}^{\neq}-\Delta G_{1}^{\ddagger}\right) / R T\right]$ 